\title{
The Very Large Array
}

Starting in 1961, NRAO scientists began the process of designing a radio telescope that could make images with an angular resolution comparable to the best optical telescopes operating from a good mountain site. In 1967, the Observatory submitted a proposal to the National Science Foundation (NSF) for the construction of the Very Large Array (VLA). The VLA proposal was for 36 , later reduced to 27 , fully steerable 25 meter diameter antennas spread over an area some $35 \mathrm{~km}$ in diameter. However, there was a competing, much simpler and much cheaper proposal from Caltech for an 8 element array of 130 foot dishes. Several NSF review committees praised the VLA concept but indicated that it was too ambitious, and recommended that NRAO further study the VLA design, and that construction of the Caltech array should begin immediately. Following a confrontational battle among proponents of the NRAO and Caltech arrays, as well as a competing proposal for a 440 foot radome-enclosed antenna proposed by an MIT-Harvard led consortium, support of the VLA by the 1970 National Academy Decade Review of astronomy led to approval of its construction.

The 1973 oil crisis and the subsequent period of excessive inflation nearly killed the fixed budget project. But under the leadership of Dave Heeschen, NRAO brought the VLA project to completion in 1980, on schedule and close to the planned $\$ 78 \mathrm{M}$ budget appropriation. The VLA has been by far the most powerful and most successful radio telescope ever built.

\subsection{BACKGROUND}

The 1957 Soviet launch of Sputnik created a widespread and frenzied concern that the US had fallen behind Russia in all matters scientific, especially in anything connected with space. In astronomy, the long tradition in optical astronomy of building large telescopes on excellent mountain sites clearly established the United States as the world's leader in observational astronomy (see e.g., 
Florence 1994). Meanwhile, as discussed in Chap. 2, radio astronomers in the US, Europe, and Australia were reporting on exciting new discoveries ranging from solar system science to cosmology. The time was ripe to review the status of US astronomy and to plan for the future growth.

Radio vs Optical Resolution In spite of the dramatic advances and new discoveries made during the quarter century following Karl Jansky's pioneering work, by 1960 radio astronomers faced two challenges to further progress. First, the angular resolution of any optical or radio telescope is determined by the ratio of wavelength to size of the telescope. Because radio wavelengths are longer than optical wavelengths by a factor of about one hundred thousand, for many years it was assumed that the resolution of radio telescopes was fundamentally limited compared with the resolution of optical telescopes. Second, while optical telescopes can produce images of celestial objects with millions of independent pixels, conventional radio telescopes typically respond to the emission from only a single area in the sky. Thus, in order to map the area of interest, radio astronomers traditionally had to make a time-consuming raster scan. In this chapter, we describe how radio astronomers developed interferometric synthesis techniques to improve the angular resolution over what is possible from any filled aperture instrument, and discuss how NRAO was able to overcome considerable opposition and technical challenges to build the Very Large Array to make images of the radio sky with resolution comparable to that achieved by the best ground based optical telescopes. In the following chapter, we discuss how radio interferometry was extended to obtain angular resolutions hundreds to thousands of times better than the best optical telescopes on the best mountain sites or in space.

Early Radio Interferometry and Synthesis Imagingl The naive comparison between the resolution of radio and optical telescopes has turned out to be wrong for three important but not widely appreciated reasons. First, because radio wavelengths are long (indeed they are comparable with every day physical scales), it is possible to build diffraction-limited radio telescopes of essentially unlimited dimensions. Second, in practice, the resolution of ground based optical and infrared telescopes has been traditionally limited not by diffraction, but by turbulence in the Earth's troposphere known as "seeing." Finally, while optical and infrared interferometers are feasible, their sensitivity is limited by the need to divide the incoming signal among two or more detectors, with a corresponding loss of sensitivity, whereas at radio wavelengths the signals can first be amplified before splitting with little loss of sensitivity.

Using their single antenna on a cliff overlooking Sydney Harbor, ${ }^{3} \mathrm{McCready}$ et al. (1947) were probably the first to recognize that the response of a simple two-element interferometer was one Fourier component of the sky brightness distribution. In their paper, McCready et al. famously noted, "It is possible in principle to determine the actual form of the [sky brightness] distribution in a complex case by Fourier synthesis using information derived from a large 
number of components." However, they went on to comment that varying the height of the cliff antenna "would be feasible but clumsy. A different interference method may be more practicable."

Wilbur (Chris) Christiansen later used Earth-rotation synthesis imaging by combining the output of multiple one-dimensional scans of the Sun. Christiansen and Warburton (1955) first formed two orthogonal phased arrays, which they used to make multiple strip distribution scans across the Sun at different orientations as the Earth rotated on its axis. They then laboriously calculated the Fourier components of each strip distribution, followed by a two-dimensional Fourier inversion to obtain a two-dimensional image of the Sun.

It would be Martin Ryle and his group at Cambridge who were later able to exploit the full power of two-dimensional Fourier synthesis imaging, which is commonly referred to as "aperture synthesis" (e.g., Ryle and Hewish 1960; Ryle 1975). By combining data from a variable spacing interferometer and exploiting the rotation of the Earth to change the orientation of their east-west baseline, Ryle and Neville (1962) obtained a two-dimensional image of a 25 square degree region centered on the north celestial pole with a resolution of 4.5 arcmin. ${ }^{5}$ This technique was informally referred to in Cambridge as "supersynthesis" and in Sydney as "Earth-rotation synthesis." However, supersynthesis using the meridian fixed parabolic cylinders was restricted to the north polar region.

The Cambridge group then went on to build the One-Mile Radio Telescope using two fixed and one moveable 60 foot steerable dishes located on a onemile-long east-west baseline. The One-Mile Radio Telescope initially operated at 21 and 73 centimeters, exploiting the changing orientation of the array due to the rotation of the Earth to sample the Fourier transform $(u, v)$ plane, giving resolutions of $25 \operatorname{arcsec}$ and 1.5 arcmin respectively (Ryle 1962). Later, the resolution was improved to 12 and 6.5 arcsec with the installation of receivers for 11 and $6 \mathrm{~cm}$ respectively. The One-Mile Radio Telescope was followed a decade later by the $5-\mathrm{km}$ Radio Telescope (Ryle 1972) using four fixed and four movable steerable antenna elements. Initially equipped to operate at $6 \mathrm{~cm}$ wavelength, and in 1974 at $2 \mathrm{~cm}$, the $5-\mathrm{km}$ Radio Telescope was able to make images with an initial resolution of only 2 arcsec and later better than 1 arcsec, or comparable to that of the best large ground based optical telescopes.

The Caltech Owens Valley Radio Observatory (OVRO) In Sect. 6.6 we described the development and the many spectacular successes of the Caltech twin 90 foot interferometer (Fig. 7.1). One may wonder why Caltech did not make better use of the capabilities of their two-element interferometer to do the same kind of full two-dimensional super-synthesis (Earth-rotation synthesis) pioneered by Martin Ryle and colleagues at Cambridge. Although Cambridge was characteristically secretive about their plans, a full description of the first super-synthesis array, the Cambridge One-Mile Radio Telescope was published in 1962 (Ryle 1962). At that time, Ryle had received funding, but 


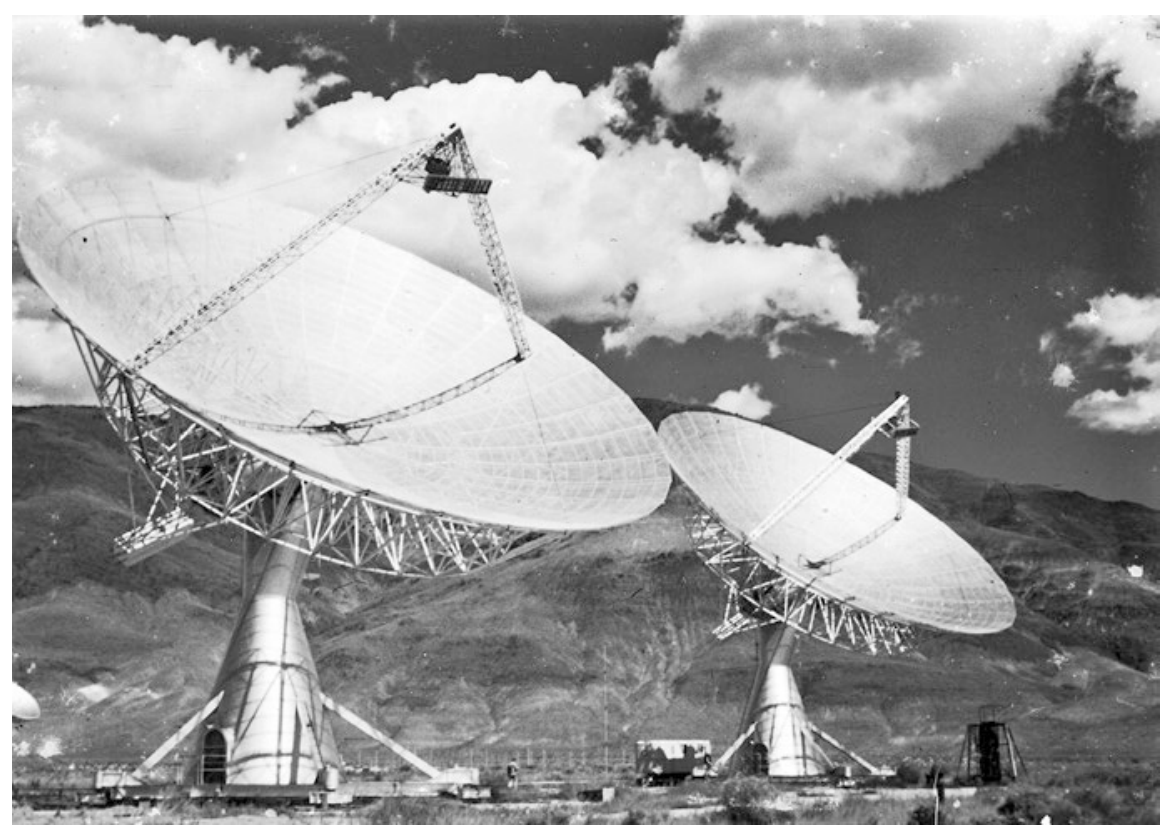

Fig. 7.1 The Owens Valley Interferometer. Credit: Caltech Archives

construction of the three-element array of 60 foot steerable dishes had not yet started. The OVRO two-element east-west interferometer was already complete and in operation by the end of 1959. By the end of 1960 the north-south baseline was also operational. This was six and five years respectively, before the first results from the Cambridge One-Mile Radio Telescope (Ryle et al. 1965). But the Caltech radio astronomers Per Maltby and Alan Moffet used Fourier inversion of their OVRO east-west and north-south OVRO transit observations of interferometer phase and amplitude to obtain only separate onedimensional strip distributions which they then used to discuss the two-dimensional brightness distributions (Maltby and Moffet 1962). Maltby and Moffet did make some amplitude-only observations at different hour angles but these data were used only to constrain the model fitting, and they never made full use of Earth-rotation synthesis and two-dimensional Fourier inversions to derive two-dimensional source images as was done later by Ryle and colleagues at Cambridge. It seems that true synthesis imaging was not implemented at Caltech until nearly a decade later (Rogstad and Shostak 1971).

Alan Moffet later claimed that, unlike Cambridge, Caltech did not have access to sufficient computing power to do a complete two-dimensional Fourier inversion of data taken at many different hour angles. While this may have been partially true, it seems that both Caltech and Australian radio astronomers were slow in appreciating the full power of synthesis imaging and were unable, or at least unwilling, to take advantage of the large digital computers available to 
them. Australian radio astronomers continued to exploit hardware solutions (e.g., Frater et al. 2017) to build high resolution radio telescopes, while the Caltech radio astronomers continued to depend on model fitting techniques to analyze their data and never made the step to full synthesis imaging with the OVRO two-element interferometer system. Nevertheless, for the first half of the decade, OVRO was clearly the most productive US radio observatory. In fact, George Swenson suggested that it was just because of their success, especially the work leading to the discovery of quasars, that the Caltech radio astronomers did not see the need to pursue synthesis imaging. ${ }^{6}$ Nevertheless, many of the students who were involved in the design and operation of the Owens Valley interferometer later went on to play major roles in the design, construction, and operation of the VLA.

The Westerbork Synthesis Radio Telescope ${ }^{7}$ Based on a suggestion from US radio astronomer Charles Seeger, ${ }^{8}$ Dutch radio astronomers, driven by Jan Oort, developed plans for a large radio telescope with dimensions of 3 to 5 kilometers and a resolution goal of 1 arcmin. Because of the anticipated large cost, Oort initiated talks with Belgium and Luxembourg for sharing the cost of what came to be known as the "Benelux Cross." At the December 1961 meeting of the European Organization for Economic Co-operation and Development (OECD) on Large Radio Telescopes, Oort (196la, b, c) presented the scientific rationale for an antenna with a resolution of the order of an arcmin while Jan Högbom (1961) and others outlined a range of design concepts. Starting with a fairly conventional Mills Cross consisting of parabolic cylinder elements working at $75 \mathrm{~cm}$ (Christiansen and Högbom 1961), the Benelux Cross project went through a series of designs and evolved to a cross composed of a hundred or more 25 to 30 meter diameter parabolic dishes working at $21 \mathrm{~cm}$ (Christiansen et al. 1963a,b). The revised array also included a single 70 meter dish to provide the missing short spacings. ${ }^{9}$ The change in operating wavelength was motivated partly by the desire to observe the $21 \mathrm{~cm}$ hydrogen line and partly because this frequency is protected by international agreement from RFI. Also, using steerable parabolic dishes instead of cylindrical parabolas opened the possibility of observing at wavelengths in addition to $21 \mathrm{~cm}$, thus facilitating spectral and polarization studies. In each of these early designs, Leiden visitors Chris Christiansen from Australia, William (Bill) Erickson and Charles Seeger from the United States, and Jan Högbom from Sweden, together with Leiden's Lex Muller, provided the technical leadership for the Benelux Cross, which they planned to locate near the Belgian-Dutch Border.

All of these early designs were based primarily on phased arrays which formed multiple simultaneous beams, although Christiansen et al. (1963a) commented on the possibility of recording the interferometer amplitudes and phases from the different antenna spacings. Högbom had joined the Leidenbased Benelux Cross group after receiving his $\mathrm{PhD}$ with Ryle's group in Cambridge, so was fully familiar with the techniques of Earth-rotation aperture synthesis. In fact, Högbom's $1959 \mathrm{PhD}$ thesis on “The Structure and Magnetic 
Field of the Solar Corona" included the first detailed description of what came to be called "Earth-rotation synthesis." According to Högbom (2003), after writing up his work on the Sun, he was embarrassed that his thesis contained a mere 78 pages, far fewer than other Cambridge radio astronomy theses, which were all longer than 100 pages. So he "fattened" up his thesis with two additional chapters, one on "The Fundamental Relations of Aperture Synthesis" based on well-known ideas, and a completely original chapter on "Aperture Synthesis Using the Earth's Rotation."

Högbom's analysis of using the rotation of the Earth to cover the Fourier transform plane was based on using a transit interferometer with a broad primary beam to allow the necessary observations at large hour angles. It was not until Högbom saw Ryle's (1962) published description of the One-Mile Radio Telescope that he appreciated the possibility of doing Earth rotation synthesis using tracking antenna elements, and immediately applied these ideas to a more practical and cost effective implementation of the Benelux Cross. According to Raimond (1996), in 1963, Högbom proposed two possible $21 \mathrm{~cm}$ east-west arrays of 28 (34) parabolic dishes spread over 1600 (3000) meters based on Earth-rotation synthesis to give a resolution of 17 (10) arcsec. Apparently Luxembourg was never a serious participant, and by 1967, with no active radio astronomers and commitments to the newly established European Southern Observatory (ESO), Belgium had lost interest in the project, which then became a responsibility of the Netherlands Foundation for Research in Astronomy (NFRA). A new, more radio-quiet, site was chosen in the northern part of the Netherlands, and the Benelux Cross became the Westerbork Synthesis Radio Telescope (WSRT).

With the loss of the expected funding from Belgium, the design was further modified to contain only ten fixed 25 meter diameter equatorially-mounted antennas uniformly spaced over $1.6 \mathrm{~km}$, plus an additional dish moveable along a 300 meter railroad track. However, when the bids came in, there were sufficient funds to build two moveable antennas. The WSRT (Fig. 7.2) was completed and went into operation in 1970, initially at only $21 \mathrm{~cm}$. Originally, each of the ten fixed antennas were correlated each with only the two moveable antennas. Since the fixed antennas were uniformly spaced, all of the available Fourier components were still recovered. However, ignoring the data from the fixed interferometer pairs resulted in a loss of sensitivity by nearly a factor of two. Although not at the time anticipated by Högbom or anyone else, after the correlator was upgraded in 1977 to include all antenna pairs, the redundant spacings turned out to be a great advantage in removing the effects of ionospheric and tropospheric phase fluctuations. ${ }^{10}$ This enhancement of conventional self-calibration techniques gave the WSRT greatly improved image dynamic range (Noordam and de Bruyn 1982).

Initially WSRT adopted a hands-off approach to observing and data reduction. The local staff supervised the observations, and the data were reduced by NFRA staff at the Leiden University computer center to produce the radio images. ${ }^{11}$ Considering the long traditions of H I research by Oort and other 


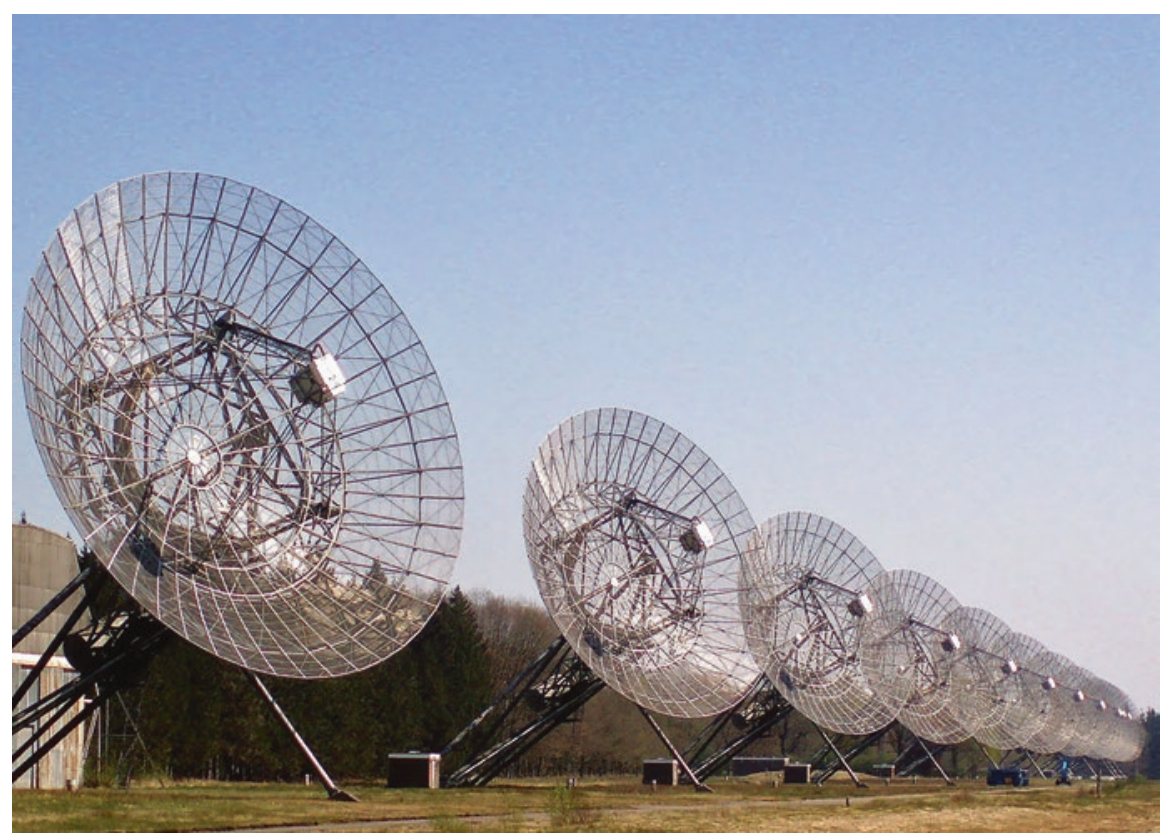

Fig. 7.2 The ten fixed antennas of the Westerbork Synthesis Radio Telescope. Credit: NFRA

Dutch radio astronomers, a spectroscopic capability was soon added to the initial continuum-only system. Over the next years the reliability, flexibility, resolution, and sensitivity of the WSRT continued to improve with the introduction of new low noise amplifiers, the addition of new observing bands at $3.6,6$, and $49 \mathrm{~cm}$, and the migration to digital electronics. Two additional moveable antennas were added in 1976, and later the baseline length was doubled to $3 \mathrm{~km}$ to improve the resolution, and the telescope continued to be used by astronomers from around the world.

\subsection{Origins of the Very Large Array and the Owens VAlley Array}

By the end of the 1950s, it was becoming increasingly clear from the exciting results coming from Sydney, Cambridge, Manchester, and Caltech that the major outstanding problems in radio astronomy required interferometers and arrays capable of arcsec resolution. As described in Sect. 3.2, as early as 1954, during the debate over the appropriate size antenna for the planned national radio astronomy facility, Bob Dicke's innovative suggestion to build an interferometer system was lost in the enthusiasm for building the largest possible antenna, but it would resurface nearly a decade later. 
The Pierce Committee Following the establishment of NRAO in 1956 and the uncertain start on the construction of the Green Bank 140 Foot Radio Telescope, NRAO Director Otto Struve suggested that the AUI Advisory Committee on Radio Astronomy request that the NSF appoint a committee to review the scientific goals of radio astronomy and the instruments needed to address these goals. The NSF responded in December 1959 by appointing a committee under the Chairmanship of John Pierce, to "l) study the present and predictable needs of radio astronomers with regard to improved instrumentation; 2) study existing and proposed instruments with regard to improved instrumentation; and 3) advise the Foundation with regard to the desirability and feasibility of constructing more powerful instruments" (Keller 1961). Pierce, Executive Director of the Bell Labs Communication Sciences Division, was a well-known engineer and expert on information theory who had conceived and promoted the first communication satellites and the national network of microwave-linked telephone relay towers. Together with Claude Shannon and Barney Oliver, Pierce had developed the first concepts of speech digitization. He coined the term "transistor" for the revolutionary device which was developed under his direction, and wrote science fiction under the name of J.J. Coupling (David et al. 2004). The other Panel members were primarily radio astronomers. ${ }^{12}$

The Panel was impressed by the newly emerging very high resolution observations coming from Jodrell Bank (Sect. 8.1), the interferometric observations at Caltech (Maltby and Moffet 1962) and Nançay (Lequeux 1962), and the demonstration of the power of aperture synthesis by Ryle and Neville (1962). With great perception, they noted that "as the Manchester group has shown, the addition of a third element gives the phases without the need for calibration." The Panel discussed, in some detail, a proposal by John Bolton for 8 -element and 16-element arrays of 200 foot dishes arranged in a Mills Cross configuration. But Bolton proposed a real-time phased array of parabolic elements, possibly making use of multiple spacings to reduce sidelobe levels, but not aperture synthesis and certainly not Earth-rotation (super) synthesis. Indeed, the later 1962 Caltech proposal for the Owens Valley Array followed the basic strategy that Bolton had presented to the Pierce Committee before he left Caltech at the end of 1960. The Panel also discussed the merits of Cornell Professor William (Bill) Gordon's plan to build a fixed spherical reflector in Puerto Rico, and anticipated the electrical and mechanical problems of designing suitable feed systems.

The Panel report, which, curiously, was published under the name of the NSF Assistant Director for Mathematical, Physical, and Engineering Sciences, Geoffrey Keller (1961), recognized the need for angular resolution of at least $\mathrm{l}$ arcmin and ultimately $\mathrm{l}$ arcsec. Although the Panel appreciated the power of aperture synthesis to obtain the needed high resolution, they correctly worried about how to achieve the instrumental and atmospheric phase stability needed to obtain 1 arcsec resolution. They recommended further "experimental and theoretical studies of aperture synthesis, antenna design, phase preservation, 
phase shifting, and stable low noise preamplifiers that that would lead to a detailed and practical plan for a radio telescope of high resolving power" and went on to suggest that "technical considerations of terrain, atmospheric and interference environment, and other scientific factors should govern the selection of the site, and convenience of access to particular institutions should be given only secondary consideration." But they also noted that "in the United States, the chief weakness of radio astronomy is not the lack of instruments or funds for instruments but a lack of radio astronomers," so the Panel recommended that universities support their most promising graduates and give postdoctoral fellowships using a combination of government and private funding. Unfortunately, it would be another decade before US radio astronomers could agree on what to build and who should build it, and yet a further decade before the Very Large Array would be completed and in operation. Meanwhile, in 1970, the WSRT began operating in the Netherlands, and in the UK, Martin Ryle and colleagues were doing exciting work with his One-Mile and, starting in 1971 , the $5 \mathrm{~km}$ radio telescopes. In Australia, Paul Wild completed his 96-element solar heliograph array in 1967 and was making impressive movies of solar radio bursts.

Early NRAO Planning At the 1961 IAU General Assembly in Berkeley, CA, Commission 40 (Radio Astronomy) discussed high resolution radio telescopes. Campbell Wade later recalled that Dave Heeschen returned from the IAU meeting enthusiastic about the potential for building an array for high resolution radio observations, and led an impromptu discussion with Wade, Frank Drake, Roger Lynds, and Dave Hogg in the Green Bank cafeteria. ${ }^{13}$ While there was apparently a lot of interest among the Green Bank staff, there was no one at NRAO with any experience in interferometry. As discussed in Chaps. 3, 4 , and 9, in the early 1960s, NRAO was focused on building a very large filledaperture radio telescope, using either a fully or partially steerable reflector or a large fixed reflector. They had even given a name to this hypothetical future project-the Very Large Antenna or VLA! Later this became the "VLAA" for Very Large Antenna Array, which finally reverted back to "VLA," but now meaning Very Large Array. NRAO and AUI were struggling to deal with the increasing problems surrounding the 140 Foot Telescope construction and the threats from Bliss to sue AUI for breach of contract regarding the 85 foot telescope project (Sect. 4.4), as well as completing the construction and commissioning of the 300 Foot Transit Telescope. Nevertheless, through the autumn of 1961 Heeschen, Wade, and others gave further thought to constructing an array of dishes. However, there was little dedicated effort until Heeschen brought things into focus on 5 March 1962 when he called a staff meeting for that same afternoon to discuss a draft development program for what he called "the very large telescope," declaring that the plan "will be submitted to NSF this week (probably tomorrow) as justification for our 1964 budget." 14

Joe Pawsey had already been appointed to succeed Struve as the next NRAO Director, and was due to visit two weeks later. Although Heeschen was only 
serving as an Acting Director until Pawsey was to take over in October, he acted boldly and requested $\$ 3$ million to be included in the FY1964 budget "for the first phase in the development of a very large radio telescope." 15 Heeschen proposed that Phase I begin by establishing the performance requirements and the antenna configuration needed to meet those requirements, followed by designing the antenna elements and electronics, studying of the effects of the atmosphere on phase stability, along with selecting a site and building a small number of antenna elements with electronics as a prototype. Regarding the site, Heeschen noted, in passing, that "Green Bank may not be suitable." Phase II, which Heeschen optimistically projected could begin in FY1965 or 1966, would be to "construct full telescope by expanding the portion built in Phase 1." As Heeschen noted in a 1991 handwritten note scribbled on a copy of his 5 March 1962 memo, "We never got the $\$ 3 M-$ but this was the formal beginning of the VLA pjt [project] \& in fact the pgm [program] outlined was generally carried out." 16

Heeschen's ambitious plan was encouraged by Pawsey during his March 1962 visit to Green Bank, although a week after he arrived in Green Bank, Pawsey's trip was abruptly terminated by his illness (Sect. 4.6). Following his surgery in Boston, Pawsey described his "high resolution project" as part of his carefully considered plans for the future of NRAO.${ }^{17}$ However, the following day, AUI President Jerry Tape and Pawsey agreed that Pawsey would not take up the NRAO directorship, but that he would stay involved in NRAO programs. It is curious that, while Pawsey's report acknowledged his discussions with Drake and Heeschen about their proposed millimeter initiative (Chap. 10), he makes no reference to any discussions about an imaging antenna array. Earlier, Pawsey had written from his Massachusetts General Hospital bed to Bill Erickson to recruit Erickson to come to NRAO to be in charge of a project to develop the necessary "equipment capable of giving pictures ... of discrete sources in the sky with sufficient resolution to show all the significant physical features."18 Pawsey sent a copy of his letter to Heeschen who distributed Pawsey's ideas about an imaging array to the NRAO Scientific Staff. Pawsey also contacted Peter Scheuer, expressing the hope that Scheuer and Henry Palmer might also come to Green Bank to examine the statistics of radio source interferometric measurements from Cambridge, Jodrell Bank, Sydney, and Caltech, and, in this way, define the needed instrument parameters. ${ }^{19}$ Erickson responded that he had accepted a position at the University of Maryland and was unable to consider Pawsey's request, but remained interested and expressed willingness to help where feasible. ${ }^{20}$ Palmer did spend a year at NRAO from October 1972 to October 1973 working with the Green Bank Interferometer.

Heeschen did not wait for Erickson or Scheuer, and asked Wade and others to assemble what was known about radio source structure and to investigate the various technical issues that would be needed to plan for the construction of the VLA. ${ }^{21}$ Some guidance on the desired array parameters was already available to NRAO from the Pierce Advisory Panel, which led Heeschen to suggest a goal of "one arcminute beam at $21 \mathrm{~cm}$ and usability at $10 \mathrm{~cm}$ to later give 
even higher resolution." For the most part, Heeschen's 5 March plan was actually implemented, but the time scale would be much longer than he had anticipated, and it would take another decade before funding was approved and nearly two decades before the VLA became fully operational. NRAO never got the $\$ 3$ million that Heeschen requested for the FY1964 budget, but by late 1962, Heeschen had been named as the NRAO Director and was ready to seriously address the construction of a large radio telescope array. In January 1963, he informed the AUI Board about the Green Bank discussions of an "array made up of 100' to 150' antennas"22 and appointed Deputy Director John Findlay to initially lead the VLA development program.

Recognizing that a major fraction of the cost of the planned array would be in the individual antenna elements, NRAO needed "the best telescope for the lowest cost" and issued a Request for a Proposal to antenna companies, soliciting design studies for "a very large radio astronomy antenna system .... [that] will consist of a number of parabolic dish telescopes." Prospective bidders were asked to determine "the relative cost of the various choices that will have to be made by the Observatory staff," which included dish diameter, upper frequency limit, polar or alt/az mount, surface accuracy, and sky coverage. ${ }^{23}$

In 1962, NRAO had no experience in interferometry. Indeed, although the Pierce Committee enthusiastically endorsed an interferometric array to advance US radio astronomy, the only place doing serious interferometry in the US was Caltech, where John Bolton, his students, and post docs had built the Owens Valley two element interferometer. Bolton, and later Gordon Stanley, served on the AUI Visiting Committee for NRAO, so there were good opportunities for NRAO to learn from Caltech experience, and in October 1962, Cam Wade was dispatched to spend three weeks at Caltech to become more familiar with interferometric techniques. It was the first time Wade had actually seen an interferometer in operation. His main reaction on returning to Green Bank was that NRAO needed to find a better way of taking data than the pen and ink tracings on chart recorders used at Caltech. Wade also visited Stanford, as well as several industrial laboratories in Silicon Valley, where he learned about early developments of fiber optic technology, but concluded that while the technology was promising, it "had a hell of a long way to go," and concluded that NRAO should "stick with cable." 24

By the end of 1963, Wade (Fig. 7.3) was able to put down on paper a basic description of the VLA. ${ }^{25}$ Assuming that the proposed array would need to be able to observe a few hundred discrete radio sources with hundreds of pixels per source and that it should not take longer than a month to observe each source, Wade concluded that they needed to build an array several miles in extent, with at least twenty 80 foot diameter paraboloids able to be placed on 106 stations arranged on a Tee configuration. The VLA that was later built had little resemblance to Wade's early plan except perhaps for the antenna diameter, which Wade later admitted was based more on the commercial availability of 25 meter diameter antennas than on sensitivity arguments. ${ }^{26}$ Dave Hogg later recollected that Wade's memo marked the real starting point of the 
Fig. 7.3 Cam Wade wrote the 1963 memo that initiated the VLA project. Later, Wade led the study leading to the choice of the VLA site on the plains of St. Agustin, and became the first Director of VLA Operations. Credit: NRAO/AUI/NSF

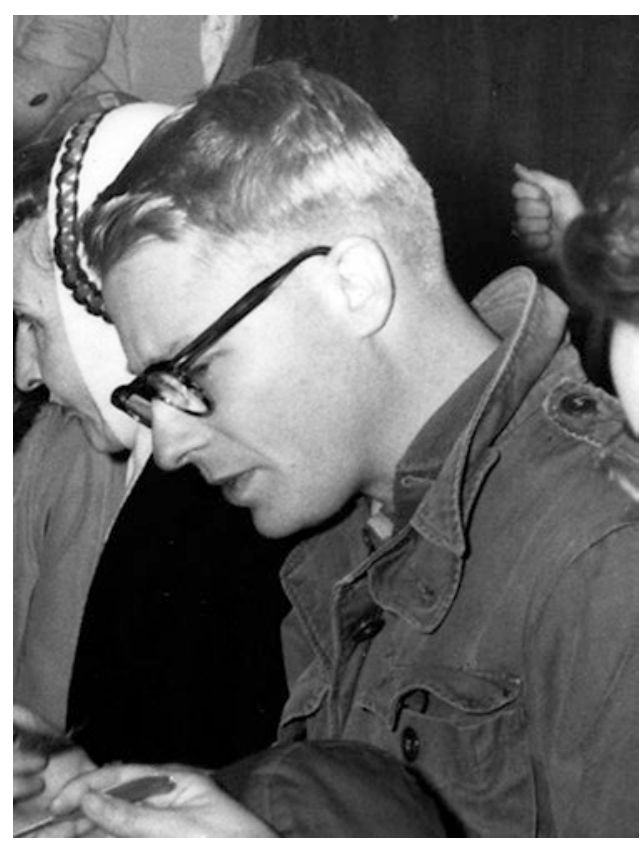

VLA project. ${ }^{27}$ A few months later, Heeschen brought the project to the attention of the AUI Executive Committee, where the acronym VLA appears to have been used for the first time for the Very Large Array and not for the Very Large Antenna, which was being pursued as a separate project now known as either the Largest Feasible Steerable Telescope or the Largest Feasible Steerable Paraboloid (Sect. 9.4). ${ }^{28}$ Assuming that the VLA design would be completed in 1967 and 1968, the schedule called for "construction to begin in FY1969 and be completed in FY1971," and that "as the VLA approaches completion, the effort on the Largest Feasible Steerable Paraboloid will be augmented." 29

The Owens Valley Array Hoping to build on their outstanding successes with the two-element OVRO interferometer, Caltech proposed adding four new 125 foot diameter antennas and an extension of the track to be used in various configurations of a phased or synthesis array. Knowing that operations support from the Office of Naval Research was becoming more difficult to obtain, Gordon Stanley sent the proposal to the NSF. ${ }^{30}$ Although Stanley and others were aware of the growing problems with the NRAO 140 Foot Telescope, they boldly proposed a polar mount for the new OVRO antennas. ${ }^{31}$ Working at a minimum wavelength of $10 \mathrm{~cm}$, the proposed array would be able to synthesize a 1 arcmin beam or work as a simple one-dimensional interferometer with 10 arcsec resolution. The proposed cost was about $\$ 5$ million. However, as early as October 1962, tensions started to build between NRAO 
and Caltech, when during a visit of Marc Vinokur to Pasadena, Caltech director Gordon Stanley expressed concern that the proposed Green Bank interferometer might compete with the Caltech proposal for enlarging the OVRO interferometer. ${ }^{32}$

The First Decade Review of Astronomy - The Whitford Report In late 1962, the National Academy of Sciences' Committee on Science and Public Policy convened what was to become the first of a series of Decade Reviews of astronomy. Albert Whitford, of the University of California's Lick Observatory, chaired the Panel on Astronomical Facilities, which was charged "to study the probable need for major new astronomical facilities in the United States during the next five to ten years, and to recommend guiding principles and estimates of cost in order that federal funds might be employed with maximum efficiency to promote advancement of astronomy in all of its branches." ${ }^{33}$

Recognizing the growing importance of radio astronomy that led to the discovery of "new and previously unsuspected phenomena," three of the six scientists on the seven-person panel were radio astronomers and three others were optical astronomers. Bruce Rule, a Caltech engineer who had been instrumental in the construction of both the Palomar 200 inch telescope as well as the two Owens Valley 90 foot radio telescopes, rounded out the committee. So although effectively half of the Panel represented radio astronomy interests, the NAS first convened a separate ad hoc committee "to guide the deliberations ... concerning the current and future needs in the field of radio astronomy." ${ }^{34}$ Some 20 radio astronomers participated in the two-day meeting held at the NAS, which was attended by Frederick Seitz, NAS President, along with other representatives from the NAS Committee on Science and Public Policy (COSPUP), the NSF, ONR, and the President's Science Advisory Committee (PSAC). This was clearly a high level meeting reflecting the perceived importance of radio astronomy as a national priority.

The two days of discussion "developed a consensus of opinion" on the need for "the largest single undertaking," recommended by the group, which "would be the construction of a large array composed of about 100 separate parabolic antennas partially steerable, each of about 100 foot diameter, with surfaces good for $3 \mathrm{~cm}$ work," 35 with an intended resolution of 1 arcmin. "A project of this magnitude," the group continued, "is obviously beyond the capabilities of a single university and naturally falls within the province of the NRAO,$\ldots$.. which would have the responsibility for the planning and the actual construction of the instrument." However, the report of the meeting also went on to discuss "a second, less expensive array with a resolution of $10 \operatorname{arcsec}$ but with higher side lobes, designed primarily for the investigation of extragalactic sources that is already funded for construction at the Owens Valley Radio Observatory (OVRO)." ${ }^{36}$ In fact the OVRO array had not been funded; it would require the next Decade Review to resolve the growing animosity between NRAO and Caltech over who would get NSF funding to build their array. 
The Whitford Committee confined its deliberations to ground-based astronomy and called attention to the discoveries of "exploding galaxies" and quasars, the better picture of the rotation of our Galaxy, the distribution of galactic neutral hydrogen, along with the solar system studies leading to the measure of the magnetic field surrounding Jupiter, the structure and temperature of the invisible surface of Venus, and an improved measure of the Astronomical Unit. Citing the study of quasars as "excellent examples of the complementarity of radio and optical astronomy," and the faintness of quasars at all wavelengths, the committee drew attention to the need for better access to large facilities at both optical and radio wavelengths. In their 1964 Report, however, the committee tempered their evaluation of very large new optical telescopes with concerns about seeing, the cost effectiveness of large apertures and corresponding ancillary instrumentation, and "the need for access to large telescopes by a much larger number of astronomers." The report recommended the construction of three optical telescopes with apertures in the range 150 to 200 inches as well as "four general purpose telescopes of aperture range 60 to 84 inches" and "eight telescopes of 36 to 48 -inch aperture." They then went on to suggest that only after the construction of three large telescopes was underway, "a representative study group be assembled to consider the problems of building a telescope of the largest feasible size." As would be the case in future Decade Reviews, reflecting the different emphasis by the radio and optical communities, the committee recommended constructing more modest-sized optical instruments in order to provide more observing time rather than instruments which would give new capabilities.

The report took a much more aggressive approach on radio astronomy. Noting that "the technical knowledge exists to build instruments that can reach beyond the thresholds of information now foreseen." the Whitford (1964, p. 19) Committee drew attention to:

- The major factor that limits the advance of radio astronomy is not particularly lack of observing time ... but rather the lack of instruments of the proper design to meet problems now recognized.

- None of the proposed or existing instruments will provide the versatility, the speed, and particularly the resolution demanded for substantial progress.

- Contrary to the situation in optical astronomy, radio telescopes have not nearly approached the ultimate limitation on performance produced by inhomogeneities in the Earth's atmosphere.

- Clearly ... no definitive knowledge of the radio sources throughout the universe can be obtained until the resolution of the order of seconds of arc is available for radio astronomers.

Although the Whitford Committee report recognized the need for "a group of lesser instruments useful in special problems and for student training," their highest recommendation for radio astronomy was for "a major high resolution 
instrument ... with a resolution of less than $10 \operatorname{arcsec}$ at centimeter wavelengths." The panel recommended "as the largest single undertaking in radio astronomy, the construction of a large array that would achieve these goals." As an example of the magnitude of the project, the Committee suggested an array of "about 100 separate parabolic antennas, each perhaps 85 feet in diameter" capable of operating "down to wavelengths as short as $3 \mathrm{~cm}$ " which they estimated could be built for a cost of about $\$ 40$ million. Characteristic of the unwarranted confidence of many preliminary cost estimates, the panel noted that "the cost is fairly predictable," since 85 foot antennas were readily available from several industrial suppliers, and that there was considerable experience in interferometry. Although the considerations about the antenna cost were probably not far off, the panel failed to recognize the true complexity and corresponding costs involved in building and using an array that would meet the astronomical requirements. In the end the VLA cost about twice the Whitford Committee estimates, perhaps not so bad considering the significant inflation that would occur before the VLA was completed 15 years later. ${ }^{37}$

The Whitford Committee noted that the complexity of the array would place the project "beyond the capabilities of a single university." However, they fell short of a full endorsement of NRAO to construct the array, only remarking that the project "falls naturally into the category of instruments that should be constructed by NRAO," and went on to specify that "means should be provided for extensive participation by scientists who are not members of the NRAO staff in the planning and development of the instrument." (Whitford 1964 , p. 52) Moreover, recognizing that it might take a decade to build the VLA, and prompted, no doubt, by Bruce Rule and knowledge of the growing ambitions at Caltech, the Committee not only recommended the funding of the "already-proposed extension" of the OVRO array to add four new 130 foot antennas along with an increase in the length of the interferometer, but suggested that "a further increase in the available equipment by a factor of two will allow useful resolutions of less than 10 seconds of arc." (Whitford 1964, p. 52) The estimated price tag for the enhanced OVRO array was only $\$ 10$ million dollars, and the panel recommended that "construction should be commenced immediately." Comparison with the $\$ 40$ million price tag and decade-long construction time estimated for the VLA positioned the Owens Valley Array and the VLA for a long and bitter conflict that would drag out for another decade, during which time nothing would be built in the United States.

Proposing the VLA Encouraged by the August 1964 Whitford Report, NRAO began serious planning for the VLA in the summer of 1964. Progress was greatly expedited by the arrival of Barry Clark on the NRAO staff only a few months later. Clark had just received his $\mathrm{PhD}$ from Caltech, where he had become an expert in radio interferometry and participated in the early design of the Owens Valley Array. For the next half a century, Clark, probably more than anyone else, was the intellectual force behind the VLA software, and arguably was the only person who understood all aspects of the VLA design. Dave 
Heeschen formally established the VLA Project, and in September 1964 he hired George Swenson (Fig. 7.4), on leave from the University of Illinois, to come to NRAO to help design the VLA. On 20 October 1965, Sander (Sandy) Weinreb came to NRAO to lead the Green Bank Electronics Division and to be responsible for the conceptual design of the VLA hardware, including the front ends and correlator. The proposed NSF FY1967 budget contained \$1 million for preliminary design of the VLA, and Heeschen later noted that this was "the first specific action taken by the NSF to allocate funds for this facility." 38

Swenson, together with Cam Wade, investigated potential sites for the VLA; Wade also investigated the needed sensitivity and antenna size. David Hogg worked on the antenna configuration, Hein Hvatum on the antennas, Sandy Weinreb and Warren Tyler on the electronics, and Barry Clark on the computing system and data processing (Heeschen 1981, p. 16). Under Swenson's leadership, by the end of 1965 the Design Group had made sufficient progress "to solicit comments, criticisms, ideas, and assistance for further work." NRAO (1965) released a preliminary design report for the VLA that described in considerable detail the status of work on the development of the VLA and the desirable properties for an instrument to address the outstanding astronomical problems of the time. ${ }^{39}$ These early specifications were:

(a) Wavelength: $10 \mathrm{~cm}$

(b) Resolution: 10 arcsec

(c) Field of view: 5 arcmin

(d) Sensitivity: 4 mJy rms

(e) Versatility to address a wide variety of problems

(f) Expandability

Fig. 7.4 George Swenson served as VLA Project Manager while on leave from the University of Illinois. Credit: NRAO/AUI/NSF

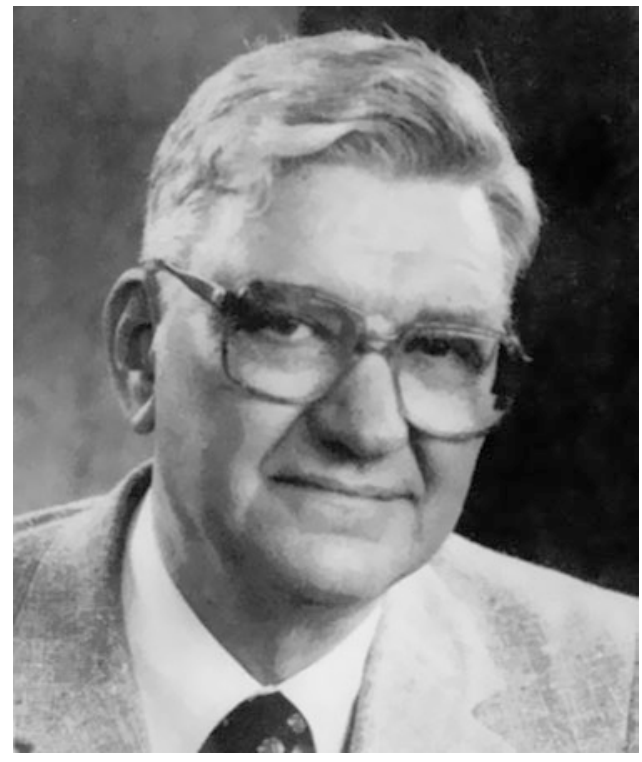


After comparing the various approaches to obtaining high angular resolution, the VLA report concluded that a correlator array based on super-synthesis or Earth-rotation synthesis ideas was the only practical way to meet the desired goals of the VLA. Various configurations including a Tee, circle, cross, and a random configuration were investigated, but Leonard Chow, visiting from Waterloo University in Canada, came up with an innovative 3-arm Wye configuration with each of the arms separated by 120 degrees. The Wye has the same comparable coverage of the Fourier transform plane as a ring, but has the advantage that the antenna elements can transported on rails or road along a straight line so that the antenna spacing can be varied, and, if later desired, the array can be extended. The proposed configuration in the 1965 report had 12 antennas spaced along each of the three arms of the Wye, with one additional antenna placed at the center. It was suggested that initially each arm would be $2.4 \mathrm{~km}$ long, which gave the desired 10 arcsec resolution at $11 \mathrm{~cm}$. Later, "as can be justified by the progress of observations ... and as funds become available, the arms of the Wye can be extended," and it was noted that, "In choosing a site, the requirement for $25 \mathrm{~km}$ arms will be considered ... to achieve l" resolution."

The NRAO report made the point that the VLA would use point sources to calibrate the baselines, rather than the laborious precision survey used by Ryle for the Cambridge One-Mile Radio Telescope. The report left open the questions of antenna size, alt-az or equatorial mount, Cassegrain or prime focus feed, Wye or Tee configuration, railroad track or road, length of the arms, type of delay system, local oscillator distribution, and single or dual polarization. NRAO proposed that the VLA use the same basic system being used for the Green Bank Interferometer (GBI), with uncooled parametric amplifiers followed by a double sideband mixer, and with each antenna pair multiplied in a correlator. There was no discussion of possibly using digital delays or a digital correlator, although it was recognized that as in the GBI, the correlator output would be digitized and fed to a high speed computer for further processing. Cautiously, the report remarked, "Cooling the amplifier to achieve low noise temperature should be avoided."

The ambitious-many felt too ambitious-NRAO VLA Report No. I indeed generated a lot of community interest, but also generated controversy. As later described by Heeschen (1996), initially,

The VLA did not enjoy much support, either in the US or in the rest of the world. The proposed instrument was considered to be unimaginative, undesirable, unneeded, technically unfeasible, far too costly, or some combination of these. It took a long time to convince the community and the NSF that was what they really wanted.

From the beginning, "the primary mission of the telescope" was considered to be "mapping of extra-galactic sources," so there was no provision for spectroscopy, which was dismissed with the remark that "the addition of line spec- 
trometer equipment to the already formidable array of data processing apparatus required for seconds-of-arc angular resolution appears to increase the complexity of the whole system to a point not consistent with the present state of the electronic art" (NRAO 1965, p. 6). This raised a lot of objection from H I observers and the growing group of outspoken molecular and maser spectroscopists.

A major challenge to the feasibility of the VLA concept came from the UK, where Martin Ryle argued that atmospheric irregularities and turbulence would introduce phase fluctuations on interferometer baselines longer than a few kilometers. While the Cambridge development of aperture synthesis and later super-synthesis had an enormous impact on the future development of radio astronomy, and in particular on the NRAO proposal to build the VLA, ironically, Cambridge radio astronomers incorrectly argued that phase fluctuations due to tropospheric irregularities would fundamentally restrict the resolution of radio telescopes to about 1 arcsec (Hinder and Ryle 1971), or about the same seeing limit achieved by optical telescopes located on a good mountain site.

The instrumental phase of the Cambridge instruments was sufficiently stable that calibration observations were needed only at the start and end of each 12 hour run. In fact, there was no capability provided in the control or data reduction software to allow calibration data to be inserted during the continuous 12 hour track. Since, at least initially, the Caltech interferometer had poor instrumental phase and amplitude stability, it was standard practice to observe a calibration source several times an hour. Unlike the Cambridge One-Mile Radio Telescope which ran under computer control, at Caltech, the pointing of the telescope was manually controlled at all times, and data were reduced by hand from chart recordings. So, even when later instrumental improvements greatly reduced the instrumental instabilities, it was natural at Caltech to extend the same calibration technique to reduce the effect of tropospheric phase fluctuations. In this way it became possible to build radio telescopes with resolution better than the nominal seeing limit. With the later development of "self-calibration," radio telescopes were routinely able to achieve resolutions orders of magnitude better than optical telescopes.

In January 1966, Heeschen informed the AUI Board that he planned to allocate $\$ 1$ million to the design of the VLA, and appointed a VLA Design Group of ten scientists and engineers with George Swenson as the Chair. ${ }^{40}$ Over the following year, the Design Group under Swenson studied various antenna configurations, explored potential sites, and, with industrial contractors, studied various antenna designs. In January 1967, NRAO (1967) sent a formal proposal to the NSF to build the VLA. The proposal called for operation at 2.7 and $5.4 \mathrm{GHz}(1 \mathrm{l}$ and $5.5 \mathrm{~cm})$ with up to $\mathrm{l}$ arcsec resolution. To achieve the desired sensitivity $(0.02 \mathrm{mJy} \mathrm{rms}$ at $2.7 \mathrm{GHz})$ and dynamic range $(20 \mathrm{~dB})$ NRAO proposed to use thirty-six 25 meter diameter antennas in a Wye configuration with each arm up to $21 \mathrm{~km}$ in length. Still, there was no spectroscopic capability planned, other than to note that the design "should not preclude the ultimate use of the instrument for line work." The estimated 
cost of construction, including $15 \%$ contingency and allowance for cost escalation over the planned four-year construction period was \$51.9 million. Annual operating costs were projected to be $\$ 1.7$ million. As noted earlier, the NRAO proposal was not without controversy. Many radio astronomers, both external to NRAO as well as within NRAO, felt that the VLA was too ambitious and too expensive. Moreover, with the increasing US budget deficits resulting from the escalating confrontation in Vietnam, there was little national interest in spending money on an expensive scientific enterprise of dubious national relevance. The NRAO budget request for FY1969 was reduced from \$24.091 million to $\$ 6.4$ million, and it was clear that there would be no VLA construction funds in $1969 .{ }^{41}$

\subsection{The Green Bank Interferometer (GBI)}

As early as December 1958, while the Tatel Telescope (85-1) was still under construction, Dave Heeschen inquired of Blaw-Knox about the possibility of putting the antenna on a railway track so that it might be used later with the 140 Foot as part of a variable spacing interferometer. Cam Wade suggested starting instead with a small two-element interferometer to (a) test methods of local oscillator and IF signal transmission, and (b) develop methods of correlating the data. Wade recognized that these questions needed to be addressed before waiting for the completion of the first two VLA antennas, which he very optimistically stated "can hardly be finished sooner than 18 months from now." 42

In January 1963, the AUI Board of Trustees approved Heeschen's request to obtain a second 85 foot antenna in order to "gain experience with interferometers." ${ }^{" 43}$ The 85-2 antenna as it was called, was essentially a clone of 85-1, except that it was mounted on a set of 96 large truck tires and could be towed by two bulldozers along a roadway. For actual observing the antenna was lowered and bolted to stations with spacings that varied between 1200 meters and 2700 meters from $85-1$, oriented along an azimuth of 243 degrees as restricted by the site geography.

The two-element Green Bank Interferometer was in operation by the middle of 1964 at $2695 \mathrm{MHz}(11.3 \mathrm{~cm})$ using a double sideband mixer with an IF band extending from 2 to $10 \mathrm{MHz}{ }^{44} \mathrm{~A}$ room temperature commercial parametric amplifier was used in front of each mixer to give a system temperature of about $125 \mathrm{~K}^{45}$ Initially all the GBI observations were recorded and reduced using strip chart recorders, but soon Wade discussed the techniques needed to find the amplitude and phase of digitally recorded interferometer data. ${ }^{46}$ Then, in December 1964, only a month after arriving at NRAO, Barry Clark refined Wade's procedure for the digital reduction of GBI data ${ }^{47}$ which was then implemented by Clark and Wade. ${ }^{48}$

The GBI had a resolution of about $10 \mathrm{arcsec}$. It met all of its design requirements and provided the interferometry experience the NRAO staff needed to pursue the VLA project. However, Caltech's Owens Valley two-element interferometer was still getting all the attention because of its exciting series of 
quasar identifications at larger and larger redshifts, new planetary results, pioneering observations of radio source polarization, and the ground-breaking investigations of radio galaxy structure (Sect. 6.6). Heeschen was anxious to get some visibility for the Green Bank Interferometer, and encouraged Wade to give a talk on his precision position measurements at the spring 1965 American Astronomical Society (AAS) meeting in Lexington, Kentucky.

Unfortunately, a large gulley located between 85-1 and the nearest 85-2 station precluded interferometer spacings less than 1200 meters. With no short spacings, the GBI had limited imaging capability, and in January 1966 Heeschen informed the AUI Board that NRAO needed a second moveable dish for the GBI. ${ }^{49}$ As shown in Fig. 6.1, a third element, 85-3, allowing baselines as short as 100 meters, was added in 1967, along with a new interferometer control building and a new observing station. Interferometer control and data reduction were handled by a DDP-116 computer. ${ }^{50}$ Starting in 1966, a portable 42 foot dish was placed at Spencer's Ridge, $11.3 \mathrm{~km}$ from Green Bank, to form the world's first phase stable radio interferometer with a baseline longer than a few kilometers (Fig. 7.5). The 2 to $12 \mathrm{MHz}$ IF signal from the remote antenna was returned over a microwave radio link operating at 1347.5 MHz, which also provided the local oscillator synchronization. John Basart et al. (1970) ran a long series of observations to study the effect of atmospheric turbulence on interferometer phase.

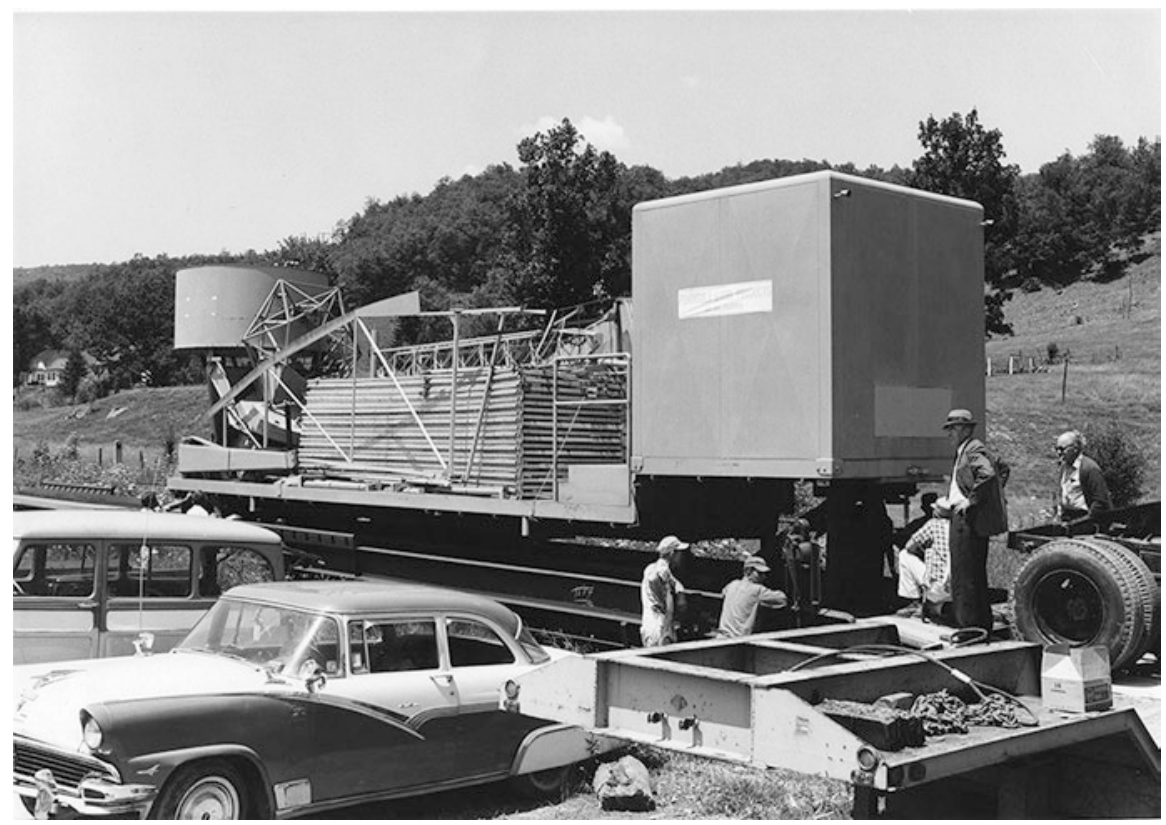

Fig. 7.5 The 42-foot antenna components arrive at Bartow railway depot. George Grove standing at the far right with his ever present pipe. Credit: NRAO/AUI/NSF 
In 1968, the GBI was further upgraded to operate at both 2695 and $8085 \mathrm{MHz}(11.3$ and $3.6 \mathrm{~cm})$ with dual polarized front ends, and the portable 42 foot dish was replaced with a 45 foot dish having sufficient precision to operate at $8 \mathrm{GHz} .{ }^{51}$ The 45 foot antenna was placed on a hilltop near the town of Huntersville, about $35 \mathrm{~km}$ from Green Bank, and was operated with an upgraded link. To avoid attenuation of the link signal from intervening foliage located in the direct line of sight, the radio link was bounced off a reflector mounted on a nearby Green Bank hill. In this way, NRAO was able to demonstrate the ability to maintain adequate phase stability over the longest baselines planned for the VLA and to make images with 1 arcsec resolution.

The 45 foot antenna was operated unattended with only a few-hour maintenance visit scheduled once a week. A decade later, the success of the remotely operated radio-linked antenna gave NRAO some confidence that it could successfully maintain and remotely operate the antennas of the proposed Very Long Baseline Array (Sect. 8.6).

The first GBI spectroscopic observation occurred in 1968, an unsuccessful attempt to detect the $\mathrm{H} 134 \alpha$ radio recombination line near $2700 \mathrm{MHz}$. Following a meeting in Green Bank in August 1968, attended by 13 NRAO and university scientists, $21 \mathrm{~cm}$ single sideband front ends, a wider bandwidth delay system, and a digital correlator were added to permit H I spectroscopy.

By 1969, with no clear prospects for VLA funding, the NRAO staff began to discuss enhancements of the GBI to improve its imaging capability by adding a fourth 85 foot dish, three 13 meter dishes, two additional observing stations along the existing roadway, and a new baseline orthogonal to the existing one. NRAO made it clear that the proposed expansion of the GBI was not a substitute for the VLA, but rather, in view of the delay in funding the VLA, it was intended as a stopgap measure to permit the kind of research not possible with the existing GBI. As described below, following the recommendations from the Greenstein Committee, NRAO received the first VLA construction funds in late 1972, and so the proposed GBI expansion never happened.

The GBI served its intended purpose, giving the NRAO scientific and technical staff the experience needed to credibly design and build the VLA, as well as exposing the broader NRAO user community to the opportunities provided by synthesis imaging. Perhaps the most important contribution of the GBI was the demonstration, using the radio linked interferometer, that although phase fluctuations initially increase with antenna separation, beyond spacings of a few kilometers each antenna is looking through essentially independent atmospheres, and the interferometer phase fluctuations remain essentially unchanged as the separation is further increased (Basart et al. 1970). Specifically, with the $35 \mathrm{~km}$ spacing of the portable dish, NRAO was able to demonstrate that it would be possible to maintain phase coherence over interferometer scales comparable to those planned for the VLA, especially since the VLA would be located on a far better site than Green Bank. The concerns expressed by Martin Ryle, who of course had great influence, were shown to be unfounded: there were no natural constraints to achieving the stated goals of the NRAO pro- 
posed VLA. Another clear result of the experience with the GBI antenna transport and the limited lifetime of its tires was that the VLA antennas should be moveable on rails and not a roadway.

An unanticipated but far-reaching contribution of the GBI came, ironically, from the deficiencies of the GBI and not from its merits. Jan Högbom, when visiting from Holland in 1967, had observed some 60 radio galaxies and quasars, but was discouraged by the poor quality of the images resulting from the large gaps in the distribution of GBI antenna spacings. As he later described it (Högbom 2003),

I found myself looking at 'dirty maps' of many sources including some calibration sources. It was then a small step to ask: if I subtract a full theoretical point source pattern, a suitably scaled and positioned 'dirty beam' from the map then there should be nothing left - unless of course there is something else out there. Often there was, and I went on subtracting. Returning to the map only the nice part the central lobe - of each subtracted pattern was a temptation I couldn't resist and it actually seemed to work. ... So CLEAN had a very simple minded beginning but in the end it turned out to be more useful than I had ever expected.

By October 1978, the VLA was in operation in four frequency bands at 1.4-1.8 GHz, $5 \mathrm{GHz}, 15 \mathrm{GHz}$, and $22 \mathrm{GHz}$, on baselines up to $12 \mathrm{~km}$. However, the NSF provided little or no VLA operating funds at this time, so NRAO closed the GBI as an NSF funded user facility, not only to free up operating funds for the VLA, but to encourage staff and visitors to use and debug the VLA. This met with some resistance, since at this time the GBI was a smoothly operating and scientifically productive instrument, whereas the VLA, not unexpectedly for a new facility, was still under construction and not straightforward to use. Moreover, there was no overlap in frequency. The VLA did not operate in the GBI bands at 2.7 and $8.1 \mathrm{GHz}$, so observations begun on the GBI often could not be completed on the partially finished VLA. But Heeschen had no sympathy for complainers. He knew the only way to get the VLA debugged was to discontinue access to the GBI and force the staff and visitors to turn their attention to the VLA.

However, until 1996, NRAO continued to operate the GBI under contract to the US Naval Observatory for their program in Earth orientation and time keeping, together with their long-running project to monitor variable radio sources at 3.6 and $11 \mathrm{~cm}$. Although the GBI was originally conceived of and was built to give the NRAO staff experience in interferometry and to prototype instrumentation for the VLA, it was an important research instrument as well for both NRAO staff and visitors. Chapter 6 discuses some of the key discoveries made with the GBI. 


\subsection{The NRAO-OVRO Wars}

Buoyed by the Whitford Committee report, and by NSF funding for the start of the first 130 foot antenna, Caltech quickly submitted a revised and enhanced proposal for the Owens Valley Array (OVA). The new proposal now included a total of eight 130 foot alt-az-mounted antennas to operate at wavelengths as short as $3 \mathrm{~cm} .{ }^{52}$ Caltech proposed completing the construction of the array by 1971 at a cost of nearly $\$ 15$ million. The new OVA proposal apparently received "excellent reviews," but in an April 1967 visit to the NSF, Stanley was informed that there was no possibility of funding in FY1968 but that FY1969 looked more promising. ${ }^{53}$ Stanley also suggested the possibility of funding only one additional antenna in 1969, a suggestion he later regretted when he learned that the OVA was already included in the NSF's planning for FY1969. ${ }^{54}$

The VLA and OVA proposals were very different. NRAO was proposing to build an elaborate national facility to be used by any qualified scientist with an appropriate program, and thus needed to be "flexible and versatile" (Heeschen 1981). This meant full sky coverage and ability to form images in one day or less. Caltech proposed a more modest instrument, with only limited public access. When first proposed, NRAO considered the VLA primarily as a continuum instrument, but recognized that spectroscopy was important, and that the design should not "preclude its future use for spectroscopy" (Heeschen 1981). The OVA put more emphasis on spectroscopy. In spite of the Whitford Committee recommendation to phase the construction of both instruments, it was clear that it would not be feasible to build both instruments, and until someone decided which would get built, nothing would get built. But, how would the decision be made? Who should decide?

The Dicke Committees By 1967 the NSF had been either unable or unwilling to fund either the OVA or the VLA. Moreover, there were other competing proposals: from Cornell for upgrading the Arecibo radio telescope to permit observations down to at least $10 \mathrm{~cm}$ wavelength, from Harvard/MIT for a large radome-enclosed radio telescope, and from a Caltech-Berkeley-Michigan consortium for a 100 meter fully steerable dish. These were all viable projects with persuasive scientific need and strong technical preparation. To consider these five major proposals, NSF convened an "Ad Hoc Advisory Panel for Large Radio Astronomy Facilities" with Princeton's Robert Dicke as the chair. ${ }^{55}$ The Panel met in Washington DC for five days at the end of July 1967 to receive testimony from each of the five proposed projects and to make recommendation to the NSF. In addition to the eight members of the Panel, more than 40 representatives of the proposing organizations, government agencies, and all three military services participated in at least some of the deliberations.

The Panel report ${ }^{56}$ issued just over two weeks after their final meeting, recommended as its clear first priority that the Caltech proposal for the eightelement Owens Valley Array "be accepted in its entirety and funded as soon as possible, with an adequate operating budget." Secondly, the Panel urged that 
the Cornell proposal to upgrade the Arecibo telescope "also be accepted in its entirety, and funded as soon as possible." The MIT-Harvard proposal for the 440 foot dish was deferred pending the outcome of the Arecibo upgrade and further engineering studies. The Caltech-Berkeley-Michigan proposal was declined. Acting on the recommendations, the Arecibo upgrade was included in President Richard Nixon's proposed FY1970 budget, but was not approved by Congress. The NSF also took the first steps toward funding the OVA, and included funds for building the first 130 foot antenna at the Owens Valley. As it turned out, the rest of the OVA was never funded, but the single 130 foot telescope had a long successful history as part of the early US VLBI program (Chap. 8) and for single dish studies.

All of the Dicke Committee recommendations carried the proviso "that at least $50 \%$ of the time available for astronomy on such facilities should be made nationally available to qualified visitors,"- a clear endorsement of an "open skies" operating philosophy, but noticeably "open" only to US-based scientists. The Panel supported the VLA concept and the need for 1 arcsec resolution. However, they argued that more work was needed to demonstrate the advantage of the VLA proposal "in terms of economy of dishes and tracks, optimization of picture resolution elements, sky coverage, observation time, and flexibility," and only recommended continued study and actual measurements to "demonstrate the feasibility of interferometric techniques over very long baselines."

Disappointed and upset with the Dicke Committee report, which appeared to "damn the VLA with faint praise," NRAO had no choice but to continue the design effort as recommended by the Committee and demonstrate that the VLA would work as claimed. To reduce the cost, the number of antennas was decreased from 36 to 27 . This resulted in an increase in the side lobe level from about one percent to about two percent. The updated design was issued in January 1969 as Volume III of the VLA proposal (NRAO 1969). Volume III included a discussion of prospective sites, a more detailed analysis of possible configurations, a conceptual design of the antenna elements and transportation system, along with the design of various components and subsystems, including the local oscillator, IF distribution, and delay systems. The proposal also reported on the successes of the GBI, including the demonstration that it is possible to maintain the required phase stability over baselines comparable to the extent of the VLA. The antenna and transporter studies, the design of the front end parametric amplifiers, the IF delay system, and the evaluation of computing requirements were contracted to industry. Most of the instrumental design work and planning for computing resources was done by NRAO scientists and engineers, to a large extent led by Weinreb and Clark respectively. As Cam Wade later explained "We took advantage of the delays to do things over again that we'd done in haste the first time." ${ }^{57}$ The new cost estimate was now only just over $\$ 32$ million.

Volume III of the VLA proposal made only brief mention of a possible future spectroscopic capability. In June 1969, Caltech countered with an 
update of their OVA proposal which focused on current spectroscopic observations at OVRO and spectroscopic applications of the OVA. ${ }^{58}$ The new OVA report also discussed the possible expansion of the OVA within the Owens Valley and to adjacent valleys as well as stating that $\mathbf{5 0}$ percent of the observing time at OVRO was being made available to outside observers, thus addressing two of the NRAO criticisms of the OVA. The proposed cost of the OVA was close to $\$ 17$ million.

Meanwhile, the NSF continued to be vague about VLA funding and asked that NRAO submit two construction plans, one for receipt of funds in FY1971 and the other in FY1974 or later. Heeschen advised the AUI Board that FY1971 "would not be unsatisfactory," but "if construction funds are postponed until 1974 or thereafter, it would be necessary to stop all design work until it was known precisely when construction funds would be available." 59 However, the NSF would not commit to the VLA or to the OVA without more explicit community endorsement.

Volume III brought the VLA design up to a point where NRAO felt the VLA was ready for final prototyping and construction. With no clear prospects for VLA construction, in 1969 Dave Heeschen dissolved the VLA Design Group and ceased further development work. With the uncertain prospects for VLA funding NRAO enthusiasm waned. George Swenson had no enthusiasm for continued fighting for the VLA and likened the situation to "scrubbing the decks on the Titanic." ${ }^{60}$ Dave Heeschen had no patience for defeatism and suggested that it was time for Swenson to return to the University of Illinois.

By 1969 none of the Dicke Committee recommendations had been funded, but the Arecibo Observatory became part of the NSF-funded National Astronomy and Ionospheric Center (NAIC). The discovery during the previous two years of pulsars (neutron stars), atomic recombination lines and interstellar (organic) molecules, along with new precision tests of General Relativity and new observations of quasars and radio galaxies, had changed the landscape of radio astronomy, which the Committee "contrasted with the tragic standstill in the funding of new facilities." Meanwhile, the 100 meter Effelsberg antenna and the 12-element Westerbork Array were nearing completion, as were new radio telescopes in India (Ooty) and at Cambridge in the UK. In view of the changes since the 1967 Dicke Committee report, the NSF reconvened the Committee "to reconsider its former recommendations, ... and to reaffirm or alter the recommendations and priorities." There was no mandate to prioritize the recommendations. Accordingly, the Panel "reaffirmed its previous recommendation" that the Arecibo telescope be improved and that the Owens Valley Array be constructed," and recommended "with equal urgency the construction of the large radome-enclosed fully steerable dish and the Very Large Array." (Dicke 1969) ${ }^{61}$ The Committee reaffirmed the recommendation that at least half of the observing time on these facilities be available to visitors and that there be sufficient operating funds to facilitate their use by non-expert observers. They also made a point of endorsing "the support of radio astronomy research and facilities at the universities." All the proposed projects 
received an enthusiastic excellent recommendation and were all deemed urgent. Such an unrealistic blanket endorsement wasn't really useful to the NSF. Only the Arecibo resurfacing would get a new start in FY1971, but there was no resolution of the VLA/OVA issue. NEROC tried an end run to fund the construction of the 440 foot dish through a special Congressional appropriation to the Smithsonian Institution (Sect. 9.5), but that plan failed in Congress.

In an attempt to resolve the stalemate, Heeschen and the NRAO staff held several discussions with Caltech to explore the possibility of jointly building an array. At an 18-19 September 1968 meeting in Charlottesville, Heeschen and OVRO Director Gordon Stanley discussed their views on some of the scientific, technical, and administrative issues facing a joint operation. ${ }^{62}$ Apparently there was sufficient common ground to agree to extend the discussions with a visit by NRAO staff to the Owens Valley. On 11-12 November, Clark, Heeschen, Hogg, Hvatum, and Wade met with OVRO's Marshall Cohen, Alan Moffet, Duane Muhleman, and George Seielstad. While there was general agreement that the two groups needed to have close and continued contact on scientific and technical issues, both sides came away suspicious of the motives and commitment of the other. Curiously, Stanley could not, or chose not, to attend the meeting, but his report to the Caltech administration emphasized the disadvantages to Caltech of a joint program. ${ }^{63}$ In early 1969, Stanley again met with NRAO scientists during visits to the potential VLA sites in Arizona and New Mexico, but returned claiming to have detected "the unshakable determination of the NRAO people to proceed with the VLA," and said that "the unanimous consensus of the [Caltech] radio astronomy group is that we do not proceed further with the attempt at cooperation on an array with the NRAO people. ${ }^{\prime 64}$

As later described by Hogg, ${ }^{65}$ there were perhaps four areas of disagreement between the Caltech and NRAO concepts:

(a) Caltech argued for a smaller number of larger dishes to facilitate calibration and to minimize maintenance. NRAO argued for a larger number of smaller elements to improve the $u, v$ coverage.

(b) The Owens Valley was too small in the east-west direction to accommodate the full extent of the NRAO VLA concept. At one point Heeschen offered to consider a joint project that would more closely follow the Caltech design, but only if the array were built on a site that allowed for future expansion.

(c) The NRAO scientists designing the VLA and using the GBI all had strong backgrounds in continuum research, while spectroscopists were mostly using the 140 Foot and 36 Foot. Noting that the H I work at Westerbork was clearly very productive, Caltech put more emphasis on spectroscopic observations. NRAO ultimately appreciated this deficiency of the VLA and adopted full spectroscopic capability for it. 
(d) Caltech did not fully buy into the visiting user concept and envisioned an operating model more like that of the Owens Valley Observatory and not the NRAO national observatory model which emphasized user support.

Desperate to find funding for the VLA, in August 1969 Heeschen, Hvatum, and Wade met in Reno, Nevada, with officials from the University of Nevada to seek their possible support in obtaining VLA seed money from the Renobased Max C. Fleischmann Foundation. The University expressed interest and offered office space, but could offer no help with persuading the Foundation to support the VLA. AUI President Gerry Tape's three-page proposal to the Fleischmann Foundation $^{66}$ was rebuffed with a curt response that the Fleischman Foundation was not interested in funding the VLA. ${ }^{67}$

The Greenstein Committee By early 1969, both NASA and the NSF, as well as the Bureau of the Budget ( $\mathrm{BOB})$, were becoming increasingly aware of the need to prioritize the many planned initiatives in both space and ground based astronomy. Following a "prospectus" prepared by the $\mathrm{BOB},{ }^{68}$ both the NSF and NASA approached the National Academy of Science (NAS) to conduct "an independent study ... which can assess the priorities of astronomy from the scientific point of view, specifically cutting across the lines of responsibility which may tend to bias the planning of individual agencies in favor of particular techniques." ${ }^{69}$ It took the NAS four months to respond with a two page proposal to form a "main committee of approximately 12 experts ... to undertake detailed planning of the study, to oversee the work of some 12 panels, and to prepare the final report" with oversight by the NAS Committee on Science and Public Policy (COSPUP) chaired by Harvey Brooks. ${ }^{70}$ The NAS moves deliberately with their studies, and expected that the report would take two years and would be delivered in mid-1971. The NSF, perhaps surprisingly, was apparently optimistic about the prospects for early construction funding, and NSF Director Leeland Haworth responded that the summer of 1971 would be marginally late to address even the NSF FY1973 budget proposal. He expressed concern that "information on astronomy is urgently needed by the Federal agencies and the Executive offices to develop astronomy support plans for earlier fiscal years. Absence of this information might slow down the U.S. astronomy program." 71 Haworth then proceeded to request "an interim preliminary report ... by early spring $1970 \ldots$ [which would] make it possible for your study to have a real impact already on the FY1972 budget and prevent any undue delays."

Jesse Greenstein from Caltech was approached to lead the study, but was less than enthusiastic. Although he had devoted most of his career to optical spectroscopy, as discussed in Chaps. 1-3, Greenstein was involved in radio astronomy almost from its beginnings. He had organized the first major international conference on radio astronomy which ultimately led to the creation of the NRAO, had convinced DuBridge to begin a radio astronomy program at Caltech, and had played a major role at Caltech in the 1963 discovery of 
quasars, although his personal (and Caltech's?) goals were unmistakably for a southern hemisphere partner for the Palomar 200 inch optical telescope (Greenstein 1984a, b; Trimble 2003; Kraft 2005)..$^{72}$

Greenstein noted that none of the recommendations of the five-year-old Whitford report had been implemented, and anticipated that the Second Dicke Committee meeting scheduled for the following month would serve to set priorities for radio astronomy. He expressed doubt on the value of a new study without some broader indication from BOB, Congress, and the President's Science Advisory Committee (PSAC), of what they wanted from a new report, whether it was an appropriate time for a new report, and he asked whether "there is any point at all in proposing large sums of money for a physical science which is not notorious for its extensive contributions to the industrial welfare, to the inner-city, to pollution etc. which seems to be the major interest of the informed Congress, and of the current administration." ${ }^{73}$

Greenstein was ultimately persuaded to Chair the Astronomy Survey Committee and agreed to provide the requested interim report. He initially approached 21 colleagues to join the main Steering Committee, only one of whom, Bernard Burke from MIT, was a radio astronomer. At their first meeting on 11-12 October 1969, the Steering Committee heard from the NSF, NASA, BOB, and Congress about their plans and expected budget levels, discussed the final composition of the committee and membership of the panels, and reviewed previous recommendations, including the Whitford report and the recently issued, but inconclusive, second Dicke Committee report.

Rather than appoint panel members who would be perceived as neutral, as was done for the Dicke Committee, Greenstein populated the Radio Panel with representatives of all the competing proposals: Dave Heeschen for the NRAO VLA, Marshall Cohen for the Caltech OVA, Frank Drake for the Arecibo resurfacing, and Bernie Burke for the NEROC 440 foot dish, and he asked Heeschen to Chair the panel. The choice of Heeschen as panel chair was not without controversy, as some committee members felt that he would bias the panel toward the VLA. ${ }^{74}$

Greenstein promptly informed Heeschen and the Panel that, "the NSF has recently taken a very strong position in favor of a major expansion in radio astronomy," and he put the Radio Panel on a fast track so that the BOB could not use the existence of the Greenstein Committee as an excuse to delay. ${ }^{75}$ Understanding that the Arecibo resurfacing would be in the NSF FY1971 budget, at their first meeting on 10 November 1969 the Radio Panel debated only the relative merits of the VLA, the OVA, and the NEROC dish. ${ }^{76}$ But they were unable to reach any consensus. If they assumed that all three projects would be funded during the next decade, the Panel argued that the OVA should be built first, but if only one project were to be funded, then the Panel favored the VLA, with only Cohen and Burke dissenting, supporting instead the OVA and the NEROC dish respectively. With so little time to meaningfully address the long-unresolved issues of priority, the Panel report did little more than endorse the Dicke Committee report that all four proposed projects 
(including the Arecibo upgrade) were important and urgent and that they be started in FY1971, although they also added a fifth project, a millimeter wavelength dish also being proposed by NRAO (Chap. 10). The Radio Panel interim report, ${ }^{77}$ dated 1 December 1969 , was approved by Greenstein and Brooks without the normal lengthy Academy approval process, and was forwarded to the new NSF Director William McElroy on 16 December. ${ }^{78}$ However, at the same time, the NAS President Philip Handler informed Greenstein, that

the whole picture developed more rapidly than McElroy or DuBridge ${ }^{79}$ expected, and the FY 1971 books are now closed. At this time nothing would be gained by dissemination of the report beyond Dr. McElroy within the Foundation ... but you can appreciate the privileged and sensitive nature of this paragraph." ${ }^{80}$

Greenstein could only reply, "We do what we can, in a rather rough world. I shall try to encourage our younger experts in the field of radio astronomy to plan for a realistic future." $" 11$

With the exception of the Radio Panel, all of the other panel chairs were members of the parent Survey Steering Committee, but the Radio Panel was represented only by Frank Drake and Bernie Burke, each of whom had their own priorities. Heeschen informed Greenstein that this was a problem, and threatened to resign if it wasn't fixed. ${ }^{82}$ Whether Greenstein was trying to correct this imbalance or was reacting to NAS President Handler's criticism that there were no committee members from the South, ${ }^{83}$ in March 1970, Greenstein belatedly asked Heeschen to join the Steering Committee. ${ }^{84}$ Again, there was some concern raised, including by Heeschen himself, that this would give NRAO and the VLA an appearance of an inappropriate advantage, but Greenstein pointed out that Heeschen was sensitive to the issue of bias, that he was a member of the NAS, and that "he is viewed by radio astronomers of the country as one of the most well-balanced and fair-minded persons possible." ${ }^{85}$ Still, having concerns that his "real or assumed bias toward NRAO could serve to work against radio astronomy in general and NRAO in particular," Heeschen only reluctantly accepted this increased responsibility. ${ }^{86}$

The four previous studies of radio astronomy priorities, the Pierce, Whitford, and two Dicke Committees had all endorsed the construction of a large radio array, but none set priorities among the modest sized university array proposed by Caltech, the more elaborate and more expensive national facility proposed by NRAO, a large steerable radio telescope of the type proposed by NEROC, or the proposed upgrade of the existing Arecibo fixed spherical reflector. Greenstein realized that the only way to get anything funded required making hard decisions about priorities, and Heeschen was determined that the NRAO VLA be the top priority.

As requested by the NSF, the Astronomy Survey Committee issued an interim report which was limited to ground based astronomy projects that might be started in FY1972 or FY1973. Having been told that there would not 
be more than $\$ 3$ to $\$ 6$ million a year for any new starts in these years, ${ }^{87}$ which excluded even a start on the high price tag VLA or OVA, the Radio Panel could not agree about the relative merits of enlarging either the Owens Valley or Green Bank interferometers. After much debate, the Radio Panel endorsed the 65 meter millimeter wave dish also proposed by NRAO as its top priority (Sects. 8.7 and 10.3) and recommended that the NSF also take over the university radio astronomy projects that had been dropped by the Department of Defense (DoD) as a result of the Mansfield Amendment. ${ }^{88}$ But after the meeting, Cohen wrote to Heeschen, "I am very concerned over the millimeter dish being put in front," and he argued instead for "two more telescopes at Owens Valley, with about $1 \frac{1}{2}$ miles of track," pointing out the "cost effectiveness of the Owens Valley Observatory" and that three Caltech OVRO graduates were on the NRAO senior staff. ${ }^{89}$ The interim report of the Radio Panel also suggested that the NSF explore the possibility of increased cooperation with NASA for very long baseline interferometry (Chap. 8).

The interim report of the parent Survey Committee included the NRAO millimeter telescope as its first priority, NSF support for all former DoD astronomy facilities, and gave an honorable mention to expanding the GBI along with other modest optical and infrared opportunities. In approving the report, COSPUP stressed that "such interim measures should not be taken as implying any decreased importance of the various items in the list provided by the Dicke panel of the NSF [and that] delay in the Dicke program will permit the Europeans to move ahead of the U.S. in this important area." 90 This caveat seems to have escaped the notice of the $\mathrm{BOB}$, as did the concern expressed by NAS President Handler about the eroding position of US radio astronomy and the "brain drain" of young American radio astronomers. ${ }^{91}$

In responding to the interim report, the NSF Director expressed his view that "In spite of the present fiscal stringencies, I am convinced that the U.S. must start on the VLA." "92 Although the OVA would have been the cheaper choice between the two array proposals, the NSF was reluctant to spend so much money on a single university facility, rather than at the NRAO where the array would serve the broader community. Moreover, the NRAO was the poster child of the NSF and NRAO had a direct link to relatively high levels at the NSF that university groups did not enjoy. The NSF was committed to making the national observatory a success and wanted to build the VLA, but they needed the endorsement of the community. This recognition that the NSF, as well as the White House Office of Science and Technology (OST), already favored the VLA helped to ultimately swing the Radio Panel to support the VLA.

However, the modest recommendations for radio astronomy contained in the interim report appeared inconsistent with the ambitious Dicke Committee recommendations and the earlier endorsement of the Radio Panel which claimed that the VLA, the OVA, the NEROC dish, and the Arecibo resurfacing were all important and were all urgent. The apparently unaggressive interim report was based on earlier information provided by BOB Director Hugh Lowerth and Philip Yeager from the House Committee on Science and 
Astronautics "that there was no possibility at all of funding for any part of the Dicke program beyond resurfacing of Arecibo for at least the next two years." 93 But as Harvey Brooks reported to Handler, "I am now told by Bill McElroy that the information given to us ... was wrong, and that the interim report and COSPUP letter have confused the issue within OMB [White House Office of Management and Budget $],{ }^{94}$ and resulted in general confusion about the priorities within the Administration... The NSF now feels that the VLA should be top priority but that the COSPUP letter undermined [their] case with OMB for the VLA," and that according to McElroy, "The difference between the interim report and the Dicke panel seems to have been deliberately used as an excuse for deferring any new starts in radio astronomy." ${ }^{95}$ Indeed, the minutes of National Science Board (NSB) Executive Session for 3-4 September 1970 show that the NSF had already included the VLA in the NSF FY1972 budget request to $\mathrm{OMB}$, although in view of the on-going Vietnam War and the then large budget deficit, it did not survive to get into the President's FY1972 budget request. Interestingly, this information was already known to NAS President Handler, since at the same time, Handler was also a member (and recent Chair) of the NSB, but he was not free to divulge this confidential information to the members of Greenstein Committee. In fact, the NSB minutes show that "The Chairman reminded the Board that all subjects discussed in Executive Session, particularly with respect to the budget, are to be treated as highly confidential." 96

Having dispensed with the interim report for modest new starts in FY1971 and FY1972, the parent Survey Committee and the Radio Panel now had to address the serious issue of dealing with the major projects: the VLA, the OVA, and the NEROC dish. Since the Radio Panel interim report had included the NRAO newly proposed 65 meter millimeter wave radio telescope in its preliminary recommendation for a 1972 new start when they had thought that any new start had to be limited to $\$ 6$ million, Heeschen was caught having to either appear to reverse that interim recommendation, or support a project that was competing with the VLA.

Greenstein stressed the need to prioritize the panel's recommendations and not just to present what might appear as a shopping list. Burke, Cohen, and Heeschen were committed to the large dish, the OVA, and the VLA respectively, to which they and their colleagues had already devoted many years of hard work and significant design funds. They were not in the mood to compromise. According to anecdotal reports, the Radio Panel deliberations were intense, with no holds barred, resulting in figurative "blood on the floor." On one occasion, when a panel member complained of the bias of the Chair toward the VLA, Heeschen walked out in disgust and threatened to resign.

After several Radio Panel meetings, it was clear that the non-committed panel members as a whole preferred one of the arrays over the NEROC dish, and it came down to choosing between the OVA and the VLA. Burke had participated in the VLA Design Group, and appreciated the potential power of the VLA. Perhaps more relevant, he was a member of the AUI Board of 
Trustees, and was sympathetic to the role of a national observatory of which he was a major user and from which he received significant support. With the NEROC dish off the table, Burke cast his lot with the NRAO VLA, and the rest of the panel went along. But there was no real agreement on whether or not the Panel should report a prioritized list which might make it more likely that at least the top one would be funded, or an unranked list which might increase the chance for two or more projects to be supported. At the last meeting of the Radio Panel in San Francisco on 18-19 February 1971, the panel agreed to stress that the entire program was needed if the US was to be preeminent in radio astronomy, but, recognizing that they could not all start at the same time, that the first new start would be the VLA. Once the Radio Panel agreed to support the VLA as the first priority for the radio astronomy, things moved very fast. Even before the Steering Committee had issued its formal report, Greenstein, together with Heeschen, went to OST to make the case for the VLA.

The formal report of the Radio Panel (Heeschen 1973), which appeared much later than the report of the parent Survey Committee (Greenstein 1972), was broad and convincing, citing the exciting discoveries by radio telescopes over the past decade that so fundamentally changed our view of the Universe. While not mentioning any specific proposals, appropriately leaving that for the NSF, the Radio Panel recommendations were nevertheless clear and unambiguous. Recognizing that the Arecibo resurfacing had already been authorized for construction, the Radio Panel recommended in order of priority the construction of (1) a large aperture synthesis array, (2) a large fully steerable parabola, and (3) a large telescope for millimeter observations (Sect. 10.3) (Findlay and von Hoerner 1972). To balance the strong support given to the NRAO VLA and millimeter telescope projects, the panel also expressed strong support for a wide range of university activities by recommending that "construction of new instruments at university facilities should continue, ... in some cases, where outstanding competence exists, major new university instruments should be provided," and said that "Support for new operations, new state-ofthe-art equipment, and maintenance of existing university facilities must be maintained at a level that will allow effective research." In support of the VLA, the Radio Panel specifically noted, "One of the most active areas of radio astronomy is the study of non-thermal sources, including quasars and radio galaxies."

Concurrent with the Astronomy Survey, the NAS also ran a physics study, led by Alan Bromley from Yale. A panel on astrophysics, chaired by George Field from Harvard, was appointed to jointly support both the astronomy and physics surveys. One of the present authors, (Kellermann), represented radio astronomy interests on the Astrophysics and Relativity Panel, which recommended that "the Astronomy Survey Committee take into account the need for a large array that can synthesize a beam of the order of seconds of arc in a reasonable period of time, for study of extragalactic radio sources" (Field 
1973a, b) so the VLA came to the parent Survey Committee blessed by two separate panels.

There were no other large "shovel ready" projects coming up from the other panels. The optical astronomers preferred more observing time and improved detectors over building more powerful new facilities, and were willing to support the VLA. ${ }^{97}$ Acceptance of the VLA as the top project for the Committee was perhaps easier in the full Steering Committee than it was in the Radio Panel. Multiple straw ballots, each with different constraints and weighting criteria, put the VLA on top each time, usually by a wide margin. According to Heeschen, ${ }^{98}$ Greenstein was initially very opposed to the VLA. Although he had played a prominent role in founding NRAO, Greenstein had come to see the relatively well-funded big national observatories as a threat to universitybased, individually-driven scientific research. ${ }^{99} \mathrm{He}$ too threatened to resign from the Committee but realized that doing so would undermine the whole study. ${ }^{100}$

Heeschen knew that the VLA had strong supporters on the Committee, and thought it better that he did not attend the final Steering Committee meeting held in Boulder, and thus it was Burke who presented the case for the VLA. Greenstein himself later explained that he was finally sold on the VLA by its expected capability to resolve the long-standing radio source count controversy, and also by the expectation that it would contribute to the broader cosmological issues facing astronomy. ${ }^{101}$ Also, Greenstein was never enthusiastic about the OVA. He came into the Survey hoping to get a copy of the Palomar 200 inch telescope in Chile. Moreover, he had little confidence in the OVRO management to construct and operate something of the magnitude of the OVA. At a higher level, within Caltech, there was more interest in enhancing the high energy physics program than in the radio astronomy program. Indeed, after John Bolton left Caltech at the end of 1960, there was only a token effort to bring in a new Director from the outside. The main competition to the VLA came from the NASA proposed series of High Energy Astronomy Observatories (HEAO) but since this was a NASA, not an NSF program, and since the cost of HEAO was an order of magnitude larger than that of the VLA, they were not really in any direct competition.

The final report of the Astronomy Survey Committee (Greenstein 1972) recommended as its top priority "A very large array, designed to attain a resolution equivalent to that of a single radio telescope 26 miles in diameter," but added, "this should be accompanied by increased support of smaller radio programs and facilities at the universities or other smaller research laboratories." A program to develop instrumentation for optical telescopes, support for the new field of infrared astronomy, a series of High Energy Astronomy Observatories, and the large millimeter-wavelength antenna received second through fifth priorities respectively. The NEROC proposal for "a large steerable radio telescope designed to operate efficiently at wavelengths of $1 \mathrm{~cm}$ and longer" was given only tenth priority and was never built. Radio astronomers would need to wait 
another 30 years before a large steerable radio telescope would be built in the US, and it only happened then as a result of a freak accident (Chap. 9).

The Survey Committee report probably ended up with a stronger endorsement of the VLA than intended by many members of the Radio Panel. Indeed after the report was released, Cohen again wrote to Heeschen that he was having second thoughts; that "the VLA will not touch on the exciting and fundamental problems: molecules and compact objects," and that he was getting a lot of negative comments from other radio astronomers. ${ }^{102}$ Nevertheless, on 22 March 1971, Heeschen, together with Cohen and other members of the radio panel, met with Edward David, President Nixon's controversial new Science Advisor, at a meeting organized by Geoff Burbidge. David noted that the group's support for the VLA "reaffirms the budgetary proposal made last year by NSF," and indicated that no further discussion was needed on this topic. ${ }^{103}$ However, as result of his abandonment of the NEROC dish and his public support of the VLA, Burke faced a formidable challenge at home from his MIT/ Harvard colleagues, who accused him of something just short of treason. ${ }^{104}$

Sensing that the tide was shifting toward the VLA, in May 1970 Stanley wrote to the Caltech management suggesting that the time had passed for the OVA. ${ }^{105}$ Trying to salvage something for OVRO, Caltech withdrew the OVA proposal and instead proposed a more modest Owens Valley Interferometer (OVI). The new Caltech proposal exploited a perceived weakness of the VLA proposal and emphasized the spectroscopic opportunities made possible by building only two new 130 foot antennas to operate together with the existing 130 foot and two 90 foot antennas. In an apparent about-face from their earlier position, the new proposal discussed the OVI as "a nationally-available facility." The proposal for $\$ 6.5$ million was sent to both the NSF and NASA, but was never funded. ${ }^{106}$ Gordon Stanley stepped down as OVRO Director in 1975 and was succeeded by Alan Moffet. Under the leadership of Marshall Cohen, Caltech shifted their emphasis to VLBI (Chap. 8).

Ironically, following the lengthy period of controversy between NRAO and Caltech, once the decision was made in favor of the VLA, it would be Caltech graduates such as Barry Clark, Edward Fomalont, Eric Greisen, and Richard (Dick) Sramek who played major roles in the final design, construction, and later the operation of the VLA. Two of the long-time VLA site directors, Ron Ekers and Miller Goss, had both worked at Caltech.

\subsection{Choosing the VLA Site}

There is no "best site" for a radio telescope, or for that matter for any telescope, as the quality of the site depends on many different criteria. The "best site" will depend on how the different criteria are weighted, and different people will weight them differently. The criteria adopted for choosing the VLA site included many of the criteria that went into choosing the Green Bank site. These included: 
1. Freedom from radio frequency interference, which meant isolation from population centers and being surrounded by mountains to shield the array from radio transmissions;

2. Low latitude in order to observe the largest part of the sky, particularly the galactic center region, and in US territory;

3 . Freedom from extreme weather conditions and earthquakes that might damage the instrument, and also low average wind speeds so as not to compromise the antenna pointing;

4. Availability of adequate power and water;

5. Proximity to a nearby town with adequate schools, cultural, and medical facilities, and access to reasonable surface and air transport.

As is the case for all radio telescopes, criteria 1 and 5 can be mutually exclusive, and the VLA had its own additional requirements. As was noted in the VLA Report No. 1, a large flat area of at least 20 miles in diameter was required to allow the individual antenna elements to be transported, and the land had to be available at reasonable cost. Moreover, it was becoming increasingly clear from experience with the GBI and at OVRO that to operate at centimeter wavelengths, clear dry skies were required, as atmospheric turbulence contributes to interferometer phase fluctuations. All the criteria suggested a site in the desert southwest. From examination of topographic maps, NRAO engineer Sidney Smith identified 14 potential sites, which he labeled Yl through Yl4. Wade later added the Plains of San Agustin in central New Mexico, which Smith had missed as it lay on the corners of four different topographic maps, but which clearly stood out as potentially an attractive site. It was labeled Y15.

Wade and Smith went to New Mexico in November 1965 to inspect the Plains of San Agustin both from the ground and the air, and to enquire about land availability. Wade was immediately impressed, and for the next five years, he considered this as the site to beat. In choosing the site for the VLA, NRAO had to consider not only the technical and logistical criteria, but a variety of social, economic, political, and environmental criteria as well. Everyone wanted to be involved-the local landowners, the politicians, concerned citizens, and of course the NSF. Although the Yl5 site stood out from the beginning as being the most desirable, 33 other sites were considered. Some of these were quickly rejected. Two were active oil drilling fields; several others were Air Force bombing ranges (Heeschen 1981, p. 11). Wade and others investigated all 34 sites between 1965 and 1971. As Wade described it, "I got to eat in lots of backwoods restaurants." 107

Much later, Wade recalled that he was troubled about discrepancies in the contour levels which described some of the potential sites that were located on different topographical maps, so before one of his trips to investigate prospective sites in the Southwest he purchased an altimeter that had been salvaged from a wrecked airplane to check site altitudes. Taking off from the Cleveland airport on the second leg of his flight back to Charlottesville from inspecting the Arizona site, Wade was playing with the altimeter to see if he could detect 
when the cabin pressure changed. Suddenly, the pilot announced that they were returning to the airport for an emergency landing. Like the other passengers, Wade, concerned about the emergency landing, hastened his exit through one of the emergency chutes, only to be taken aside by FBI agents for questioning. Apparently, spotting Wade fooling with his altimeter, another passenger notified the flight crew that Wade was about to set off a bomb. The plane set off again, but only after a long delay, and Wade was not the most popular passenger on the flight. ${ }^{108}$

To the extent possible, NRAO tried to keep the search process quiet to avoid possible political interference and land price speculation. Unlike in Green Bank, where the Observatory land was privately held, the area chosen for the VLA was mostly federal and state owned land, but was leased to private ranchers who were very protective of their grazing rights. Fortunately, Wade had grown up on a farm in Kentucky and knew how to talk to farmers and ranchers without alarming them and without the local politicians getting too involved. After multiple visits, Wade, often accompanied by NSF or AUI staff, managed to satisfy the ranchers that the VLA would not harm their ranching interests.

Of 34 potential VLA sites, NRAO let contracts to a civil engineering firm to study seven sites ${ }^{109}$ for ground stability, drainage, the suitability of underground water for drinking, suitability of soil content for construction, etc. While Wade continued to prefer the Y15 site, there were strong arguments for the Y23 site which was close to Tucson and close to where NRAO was already operating its 36 Foot Telescope (Sect. 10.2) and also close to the Kitt Peak National Observatory. But according to Wade, the Y23 site was subject to flooding, had a large rattlesnake population, and was too close to potential interference from Tucson. ${ }^{110}$ Y27, the site near Marfa, Texas and the McDonald Observatory, was considered by some to be attractive as it was in Texas, the home state of then President Lyndon Johnson. In 1967, the Plains of San Agustin became NRAO's proposed site, but it was kept quiet until 1971 when it was clear that further progress on the project depended on developing the site. This meant going public with disclosing the Plains of San Agustin as the preferred site, and NRAO (1971) submitted Volume IV of the VLA proposal to the NSF in December. Volume IV described the site selection process, discussed the relative merits of seven acceptable sites, the reasons for rejecting the remaining 27 sites, and the merits of the Plains of San Agustin as the preferred site for the VLA.

All astronomers think they are experts on telescope site selection, and nothing is ever more controversial in any big telescope project than choosing a site. The VLA was no exception. In submitting Vol. IV to the NSF, Heeschen's covering letter succinctly summarized the choice of Y15 in terms of its elevation, level ground, drainage, accessibility, and cost, concluding with "The site Y15 in the Plains of San Agustin is remarkable, and is perhaps uniquely suited to the requirements of large radio astronomy arrays." 111 Although Vol IV of the VLA Proposal probably gave more detail and more extensive justification for the VLA site selection than for any previous telescope project, the NSF needed reassurance before approving the selection of the site, and asked the 
National Academy of Science to review Vol. IV, to advise on the adequacy of selection criteria, to review the analysis of the site selection data, and to assess the conclusions. The NAS sent Vol. IV, along with Heeschen's cover letter, to C. Mayer (NRL), E.M. Purcell (Harvard), J.R. Pierce (Caltech), R.B. Leighton (Caltech), and R.N. Bracewell (Stanford), requesting their advice. All responded positively endorsing the methodology and the choice of the Plains of San Agustin, but one reviewer could not resist the opportunity to question whether or not the VLA was worth the huge cost and suggested that some of the money could better be spent on VLBI (Chap. 8). ${ }^{112}$

Work on the site began in 1974. Even though most of the land was owned by the state or federal government, gaining access was not straightforward. It took more than seven years to complete the paper-work to give the NSF the right-of-way through one parcel of land owned by the Department of the Interior. Each of the ranchers who owned land near the ends of the three arms brought suit against the government condemnation of their land, and the suits had to be settled by a court appointed commission, costing the project another $\$ 200,000$. One of the ranchers, who owned land near the end of the north arm, objected to the encroachment on a piece of land that he had developed for irrigation and farming. Unable to negotiate a mutually agreeable settlement, the north arm of the VLA was shortened to $19 \mathrm{~km}$, and so is $2 \mathrm{~km}$ shorter than the other two arms. Only one square mile of land, housing the control building, cafeteria, dormitory buildings, and the Antenna Assembly Building, was actually purchased for the VLA; the three strips of land, 300 feet on each side of the baselines, are leased.

New Mexico state law requires that land used for any new project be inspected by a state-licensed archeologist for evidence of historical land use. A preliminary survey by a New Mexico State University archeologist disclosed evidence of ancient habitation near the end of the planned array's southwest arm. The site was on land owned by a local rancher who refused admittance to the site until a court order rejected his claim (Lancaster 1982). The state of New Mexico, the NSF, and the Department of the Interior all turned down applications to fund the required excavation, and the VLA project had to pay almost $\$ 100,000$ for the archeological work, which uncovered more than 3,000 artifacts dating back as much as ten thousand years (Beckett 1980).

\subsection{BUILDING THE VLA}

Selling the VLA to the radio astronomy community, to the NSF, and to Congress took a decade, but this was only the beginning. It would take nearly another decade to address the multitude of managerial, funding, technical, and logistical challenges facing NRAO and the NSF. In the spring of 1971, when it first appeared that the VLA would be funded, Heeschen appointed Hein Hvatum, the NRAO Associate Director for Technical Services, as the new VLA Project Manager. Heeschen declared his own work finished and left for a wellearned six-month Caribbean sailing trip with his family, leaving Hvatum in 
charge. Hvatum then led a detailed review of the design of the antenna and other instrumentation. The 1960s were not only a period of rapid discoveries in radio astronomy, but one of major technical advances. Cryogenically cooled low noise amplifiers, largely developed at NRAO by Sandy Weinreb and his staff, greatly improved the sensitivity of radio telescopes, although there remained reliability issues. Digital signal processing had replaced chart recorders and analogue electronics, and astronomers were becoming more comfortable with large scale computing machines. The rapid scientific and technical advances represented both opportunities and challenges. In 1971, astronomers expected more from the VLA than they did when it was first discussed in the 1965 report, and even the 1969 proposal was technically obsolete. But no one had ever simultaneously operated 27 cryogenically cooled receivers. Indeed, it was often a challenge in Green Bank to keep a single cooled receiver operational for more than a few days at a time. The VLA goal of 10 arcsec resolution was replaced by $l$ arcsec, but the dynamic range requirement was modestly set at only 50 to 1 corresponding to maximum sidelobe levels of the order of two percent, or comparable to that of a carefully illuminated parabolic dish.

Following the Congressional approval of the VLA project in August 1972, the NSF made \$3 million available in November 1972 for VLA design and prototyping. The final antenna configuration was based on a total of 28 antennas, nine along each of the three arms plus a spare, so that at any given time one antenna could be scheduled for routine servicing and possible installation of new receiving equipment. Four configurations of the antennas were proposed to vary the resolution and field of view. The four antenna configurations provided maximum arm lengths of $600 \mathrm{~m}, 1.95 \mathrm{~km}, 6.4 \mathrm{~km}$, and $21 \mathrm{~km}$, and became known as the $\mathrm{D}, \mathrm{C}, \mathrm{B}$, and A configurations respectively. Along each arm, the spacing of the nine antennas was concentrated toward the center and followed a power law distribution of spacing that minimized the total number of stations required.

Electronics Division head Sandy Weinreb did most of the system design for the VLA instrumentation. The Green Bank Interferometer (GBI) operated in only two bands with concentric feeds, and the initial proposal to build the VLA was based on a similar system. Due to satellite interference near the $11 \mathrm{~cm}$ band, the primary VLA band was shifted to $6 \mathrm{~cm}$ with additional bands at $18-21 \mathrm{~cm}, 2 \mathrm{~cm}$, and $1.3 \mathrm{~cm}$. Each receiver first-stage was mounted on separate circularly polarized feeds located on a 2 meter diameter ring centered on the vertex of the dish. An asymmetric secondary reflector at the Cassegrain focus was rotated to illuminate each feed and direct the beam along the electrical axis of the telescope. The $6 \mathrm{~cm}$ receiver was conventional and included a parametric amplifier followed by a mixer and IF system. For the $18-21 \mathrm{~cm}$ system, the signal was up-converted to $5 \mathrm{GHz}(6 \mathrm{~cm})$ and the $6 \mathrm{~cm}$ paramp used as the second stage. In order to properly illuminate the $18-21 \mathrm{~cm}$ subreflector and to keep the feed from being prohibitively large, a dielectric lens was placed in front of the feed. The 1.3 and $2 \mathrm{~cm}$ mixers also used the $6 \mathrm{~cm}$ paramp as the second stage. Although the addition of the 1.3 and $2 \mathrm{~cm}$ bands 
improved the resolution to 0.1 arcsec at the shortest wavelength, this cost effective arrangement resulted in relatively poor sensitivity at both 1.3 and $2 \mathrm{~cm}$. At each band there were two independent receivers, one each for left and right hand circular polarization. For each polarization, the $100 \mathrm{MHz}$ IF band was split into two $50 \mathrm{MHz}$ bands, which was the largest that the digital electronics of the era could accommodate.

In order to optimize the aperture efficiency, the primary and Cassegrain secondary reflectors differed from their canonical parabolic and hyperbolic shape (Williams 1965). To evaluate the planned fixed position 4-feed system, the Green Bank 140 Foot Telescope was converted to a Cassegrain optics employing a rotating asymmetric secondary reflector. Unfortunately, the Green Bank prototype did not uncover a problem caused by the offset feed geometry, which resulted in the two circularly polarized beams being displaced by 0.06 beam widths (Napier and Gustincic 1977). By the time the problem was discovered, six systems had already been purchased, and it was decided not to implement any changes.

Although NRAO had experienced considerable issues with the reliability and stability of cryogenically cooled receivers on the Green Bank 140 Foot Telescope, Weinreb made the bold decision that in order to obtain the best sensitivity he needed to use cooled parametric amplifiers on the VLA front ends. There were initial reliability issues with the VLA front ends, but by the end of the construction project Lancaster reported that "reliability was no longer a problem" (Lancaster 1982). Ultimately the $5 \mathrm{GHz}$ paramps and mixers were replaced by separate cooled Gallium Arsenide Field Effect Transistor (GaAsFET) amplifiers for each band which gave lower noise, better stability, and improved reliability.

One of the key technical innovations employed during the construction of the VLA, was the use of the newly-developed low loss $\mathrm{TE}_{01}$ mode circular waveguide to carry the IF signals back from each antenna. The same waveguide carried the common local oscillator reference signal from the central laboratory to each antenna and the extensive monitor and control signals to and from each antenna and the central control building. ${ }^{113}$ In the 1967 VLA proposal, the signals were to be transmitted by conventional coaxial cable, but to compensate for the attenuation over the long baselines extending up to $27 \mathrm{~km}$, expensive and perhaps unreliable amplifiers would be needed every few kilometers to maintain the needed signal strength. It was felt that optical fiber technology was not sufficiently well developed at the time to be used for the VLA. Weinreb became aware of the new low loss circular waveguide that had been developed to replace the microwave relay towers then in use to support the AT\&T national telephone network. However, when they went to the NRAO business office to get approval to buy the waveguide, Weinreb and Hvatum had to admit that the waveguide was being manufactured only in Japan. Moreover, the Japanese plant where the waveguide was being fabricated 
was scheduled to be closed. In order to procure the waveguide needed for the VLA, NRAO only had a month to place the order. NSF approval was straightforward, but the US Department of Commerce was not so easily convinced why such a large government contract should go to Japan, especially without an open bidding process.

No one at NRAO had any experience in using the new circular waveguide. The loss resulting from inserting couplers at the many antenna stations in the inner few kilometers of the array meant that there would not be enough signal to reach the outer antennas. ${ }^{114}$ Moreover, standing waves set up by the couplers threatened to generate spurious propagation modes in the waveguide. After a five-year effort, NRAO solved the problem with the invention of a new coupler with a low insertion loss (U.S. Patent No. 4025878). Also, as used by Bell Telephone, the waveguide was mounted on steel springs attached to an outer steel pipe. NRAO wanted to save money and directly buried a $1.25 \mathrm{~km}$ test section at the VLA site to evaluate the long-term stability. Although the attenuation of the waveguide increased significantly over the next few months, it ultimately stabilized and the decision was made to directly bury the waveguide at least one meter deep ( 3 feet) along each of the VLA arms without using any protective enclosure (Fig. 7.6). According to Lancaster (1982), because the incremental funding prevented placing a single order and there was only one manufacturer, the Sumitomo Corporation, which was located in a foreign country, "the procurement of the waveguide was one of the most difficult actions of the VLA construction." Although the test section was obtained at a cost of $\$ 32$ per meter, subsequent asking prices jumped to $\$ 79$ per meter, but after negotiations were reduced to keep the waveguide procurement within budget.

Another important design change from the original proposal, made possible by the rapid development of digital signal processing technology, was to build a digital delay-multiplier system based on custom designed Application Specific Integrated Circuits (ASICs) to process the four $50 \mathrm{MHz}$ IF bands, two in each circular polarization. To compensate for the delay of up to 140 microsecs in the differential path length from each antenna, the digital delay system needed to maintain an accuracy of better than two nanoseconds across the $50 \mathrm{MHz}$ band. ${ }^{115}$ This complex digital system included a test and replacement capability to automatically detect component failures and replace failed components with spare units. However, the actual implementation of all four IF bands had to be deferred as the initial computer system was not adequate to handle the full data load. Although a spectral line capability was not included in the original proposal, the VLA correlator that was finally built employed a technique known as recirculation, whereby an increased number of frequency channels could be obtained at the expense of limited bandwidth. ${ }^{116}$ The VLA correlator was a large digital system operating at $100 \mathrm{MHz}$ and included 13 racks of NRAO developed hardware containing 85,000 integrated circuits. It was made feasible by using two custom developed integrated circuits which reduced the number of multilayer circuit cards from 864 to $156 .{ }^{117}$ In simple terms of megaflops, 


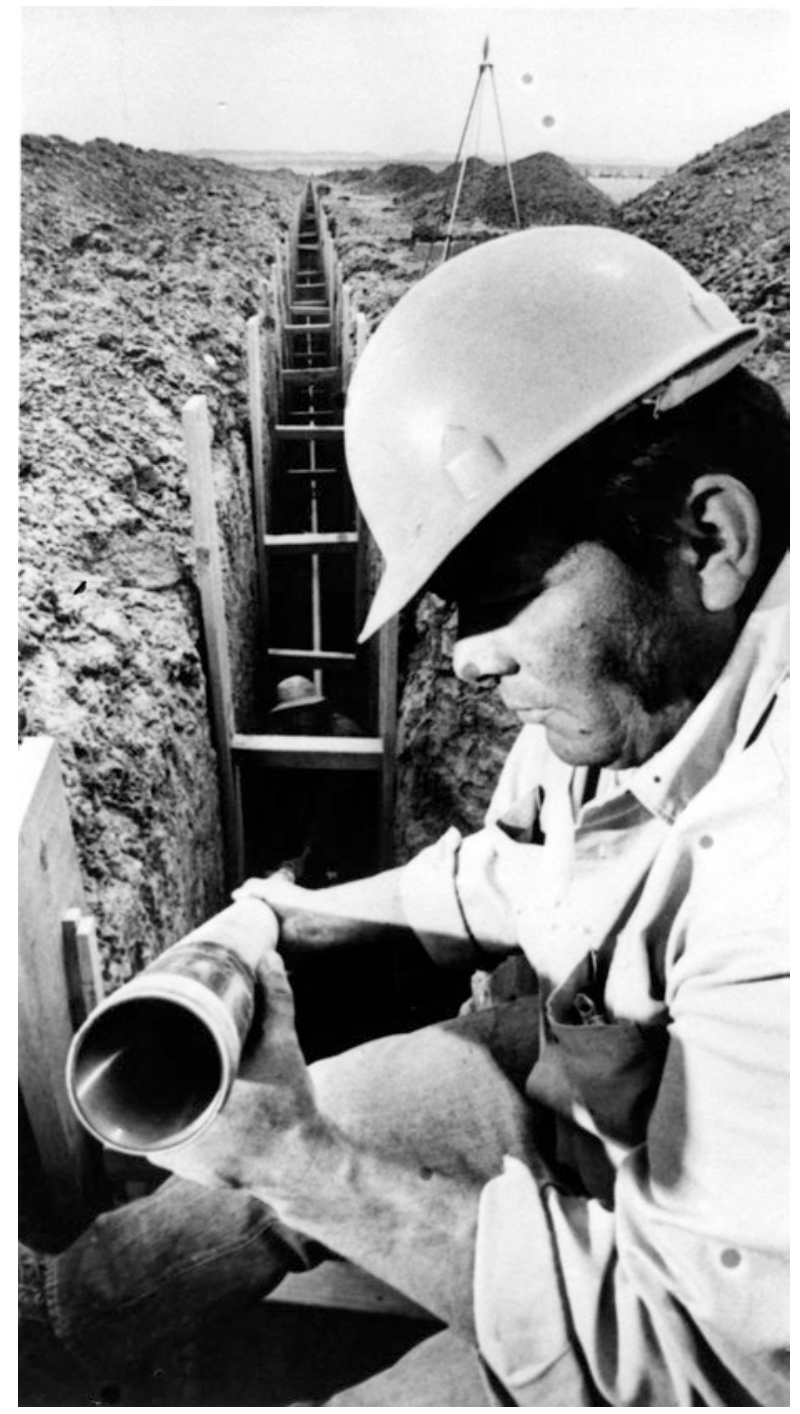

Fig. 7.6 The $\mathrm{TE}_{01}$ mode circular waveguide was buried alongside the railway track to transfer the local oscillator and IF signals between the central control building and each antenna. Credit: NRAO/AUI/NSF

the VLA correlator rivaled the most powerful general purpose computers then available.

A particularly challenging area, the computing hardware and software, was divided into two areas. Real time control of the VLA, data acquisition, and data processing were assigned to the "synchronous" system comprised of a series of Modcomp II mini computers, and was designed to have minimal real time 
interaction with the VLA operator or the observing scientist. The "asynchronous" system included off-line data editing, calibration, and processing and imaging that was initially implemented in a Digital Equipment Corporation DEC 10 mainframe computer. Recognizing the enormous challenge that the VLA image processing presented, NRAO investigated the feasibility of using analogue optical imaging processing. ${ }^{118}$ An internal committee appointed by Heeschen reported that the optical system could give better dynamic range for about the same price, but as the risk was higher, they fortunately recommended the digital processor, which was initially implemented in PDP 11/40 and PDP 11/70 mini-computers and an array processor. The original PDP 11 s were later replaced by several more cost-effective and popular VAX 11-780 machines, including one in Charlottesville, and later by Convex $\mathrm{Cl}$ mini-supercomputers. Robert (Bob) Hjellming ${ }^{119}$ led the development of the asynchronous software system and Barry Clark the synchronous system, but they were supported by a growing team of programmers along with a growing software budget. In order to take advantage of the rapid growth in computing power, the initial hardware acquisition was limited to that needed to handle only the 10 antenna continuum system, with the intention of acquiring the rest of the computing hardware closer to the end of the VLA construction. In the end, this approach gave the best computing power for the money, but severely limited the use of the partially completed VLA. In part, this was the result of the excellent VLA sensitivity and an antenna configuration that could give reasonable images even for short "snapshot" observations lasting only a few minutes rather than 8 to 12 hours. This meant one could observe a hundred or more sources per day instead of the planned two to three sources, with a corresponding increase in the computing load. Moreover, the VLA proposal assumed a relatively straightforward single data pass of gridding and Fourier transform, but the use of deconvolution techniques and self-calibration led to multiple passes and an interactive data reduction process. NRAO scientists initially assumed that due to the large number of interferometer baselines deconvolution would not be needed for VLA data. Attempts to develop a so-called "pipeline" combination of hardware and software lasted over a decade, but were never satisfactorily implemented. ${ }^{120}$

Finding the Money The FY1973 Congressional Appropriation Bill HR 15093 including initial funding for the VLA was signed by President Richard Nixon on 14 August 1972. Cam Wade recollected that when he learned Nixon had approved the VLA, Heeschen's mixed response was, "We've wanted this thing so long, and now we are getting it from a crook." ${ }^{\prime 21}$ The original NRAO plan called for a one-year design phase followed by a four-year construction plan at a total cost of $\$ 63$ million. However, the NSF wanted to limit funding to not more than $\$ 10$ million per year to minimize the impact of the VLA construction on other NSF programs. A new construction plan was negotiated, with the first year for final engineering design and prototyping funded at $\$ 3$ million, followed by a constant funding level of $\$ 10$ million per year, which stretched the construction over a period of nearly eight years. This not only delayed the 
start of full VLA operations, but the extended production schedule increased the cost due to the then high level of inflation, the loss of quantity discounts for large purchases, as well as the need to maintain the administrative, scientific, and technical support structure over a longer period of time. With an assumed rate of inflation of $6 \%$ a year, the total cost projection increased to $\$ 76$ million. As it developed, the extra time and increased funding turned out to be a blessing, as it allowed time for prototyping, testing, and, where necessary, design changes with a minimum of retrofitting. Although the VLA was technically state-of-the-art, because most of the construction cost was in straightforward areas such as antennas and railway track, it was felt that the budget plan was sound, and "NRAO was determined to build the VLA on schedule and within budget" (Heeschen 1981, p. 31). However, as described below, the actual rate of inflation became much higher, which resulted in continual modifications to the construction plan and threatened the successful completion of the VLA.

The FY1973 NSF budget passed by Congress included the requested \$3 million for VLA design and prototyping, but funding for the first year of construction hit a roadblock in Congress. ${ }^{122}$ The House of Representatives Science and Astronautics Authorization Committee included the planned \$10 million for VLA construction as part of the NSF's total \$610 million authorization bill for FY1974. However in the House Appropriations Sub-Committee for Housing, Urban Development, and Independent Agencies (HUD), Representative George Shipley (D-Illinois) commented, "The stars will still be shining in 20 or 30 years, but pollution is going to be a heck of a lot worse in 20 to 30 years." 123 Subsequently, the Appropriations committee report stated, "Although this committee approved the initial funding for this project in fiscal 1973, it now feels that that in view of general budget constraints and other earthbound National Science Foundation priorities, the VLA can be deferred," and VLA funding was eliminated from the House NSF Appropriations bill. Coincidently, as reported in the Wall Street Journal, ${ }^{124}$ the Authorization and Appropriations bills reached the House floor and were each passed on the same afternoon. With no appropriation, the authorization was meaningless. The VLA was saved when New Mexico Governor Bruce King found himself on the same plane with Senator Joseph Montoya (D-New Mexico) where they discussed the VLA problem. Montoya was a member of the Senate Appropriations Committee and brought the VLA problem to the attention of the HUD subcommittee chair, Senator William Proxmire (D-Wisconsin) of Golden Fleece fame (Sect. 5.5), and other sub-committee members. With Proxmire's support, the Appropriations bill passed by the Senate included the full \$10 million for the start of VLA construction. The House-Senate Conference Committee, as is typical in such situations, split the difference, and the final FY1974 Appropriations bills containing \$5 million for the start of VLA construction passed both houses without discussion. But this reduced funding caused yet another redrafting of the construction plan and increase of the projected project cost to $\$ 78.2$ million. At the request of the NSF, dozens of other funding arrangements were prepared over the course of the project, with 17 alone in FY1974. 
But there was still another hurdle to overcome. The NSF had to be convinced that the VLA as designed was feasible and could be built for the planned \$63 million, so they contracted with the Stanford Research Institute (SRI) to do a feasibility study of the proposed VLA. The charge to SRI was to (1) determine the proposed system feasibility and ability to meet specifications in the light of existing technology, (2) confirm the cost and time schedules for the construction, development, and operation of the system, and (3) evaluate the method for managing the VLA Project proposed by AUI. SRI convened an ad hoc committee that did not include any astronomers. The committee met five times over a period of three months. Their report ${ }^{125}$ was very favorable, but they expressed concern about whether NRAO's informal management style would be appropriate to a complex construction project such the VLA. The report confirmed that (1) the VLA was technically feasible, (2) the cost had been accurately estimated by NRAO, (3) the time for construction could be as short as four years, (4) NRAO's technical competence was confirmed, and (5) the proposed project management "is generally good, but could be improved."

VLA Construction ${ }^{126}$ Instead of bidding the entire project to build the VLA to a single contractor, NRAO decided to act as its own prime contractor to minimize the cost and to maintain tight control over the construction. Following the approval of the VLA by Congress in August 1972, Jack Lancaster joined NRAO two months later as the VLA Project Manager and NRAO Assistant Director (Fig. 7.7). Prior to coming to NRAO, Lancaster had been the Chief Project Engineer at Brookhaven, where he oversaw the construction of the major reactors and accelerators. In November 1972, the VLA Construction Project was organized as a Division of NRAO with Hein Hvatum retaining responsibility for the overall technical design (Heeschen 1981, p. 31). Groundbreaking on the Plains of San Agustin took place on 4 December 1972. In April 1973 Lancaster opened an office in Magdalena, New Mexico, about 20 miles from the center of the VLA site. Over the next eight years, Lancaster, Hvatum, and Heeschen expertly guided the VLA project to its successful completion in January 1981, on time and officially within the 1972 revised budget.

As with other radio arrays, the largest single cost item for the VLA was for the antennas. The Request for Proposals (RFP) for the antennas was based on an in-house design and cost estimate led by NRAO engineer Bill Horne. Reflecting the increasing interest in going to shorter wavelengths, the antennas were specified to have a surface accuracy of $0.75 \mathrm{~mm} \mathrm{rms}$ and pointing accuracy of 2 arcsec, sufficient to permit observations at wavelengths as short as $1.3 \mathrm{~cm}$. NRAO estimated that the antennas would cost $\$ 19.3$ million. The bids ranged from a low of $\$ 16.8$ million by E-Systems Inc. to a high of $\$ 31.8$ million from the Collins Radio Company. Following evaluation of the business and technical aspects of the proposals and discussions with each of the potential vendors, NRAO received five "Best and Final" proposals. In October 1974 NRAO signed a contract for the fabrication and construction of 28 antennas 
Fig. 7.7 Jack Lancaster, VLA Project Manager who oversaw the VLA construction. Credit: NRAO/AUI/NSF

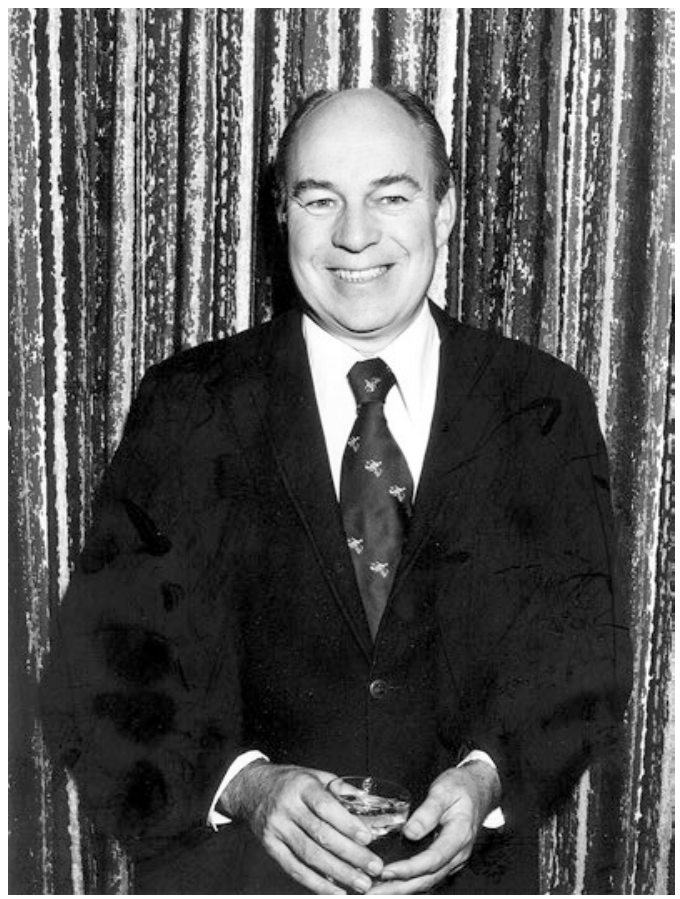

with the Dallas, TX-based firm E-Systems, Inc. The E-Systems contract also included an Antenna Assembly Building to facilitate the construction of the antennas, the installation of instrumentation, as well as ongoing antenna maintenance and repair. ${ }^{127}$

Since the fabrication and erection of the antennas was planned to be stretched out over a number of years, the contract was complex, since there is no guarantee from year to year that Congress will appropriate the needed funds. During FY1974, E-Systems completed the engineering design, and the first two prototype antennas delivered in 1975 met all specifications. The antenna contract contained options for NRAO/AUI to purchase a predetermined number of antennas each year at a predetermined fixed-price that increased each year to allow for an anticipated six percent annual inflation. This lead to the first serious problem in the VLA construction program.

Due to the oil crisis resulting from the OPEC oil embargo following the 1973 Yom Kippur War, and the abandonment by Richard Nixon of the US Gold standard and subsequent dollar devaluation, the 1970s experienced a period of extreme inflation. Within eight months the cost of steel doubled. On 6 January 1975, E-Systems notified NRAO/AUI that they were no longer able to meet their contractual fixed-price obligations. It was apparent that any attempt to enforce the predetermined prices would result in bankruptcy, leaving NRAO with no path to secure the antennas. After lengthy negotiations, it was agreed that NRAO/AUI would advance the funds to purchase the steel for 
all of the remaining antennas, which would help mitigate the impact of the high national inflation, and that E-Systems would deliver the completed antennas at the previously agreed price, but on a faster schedule. However, this meant that by spending an unplanned large fraction of the limited NSF annual funding on the antennas, the instrumentation of the antennas was delayed and the antennas were not available for commissioning or scientific observations at the planned rate. ${ }^{128}$

The less than satisfactory experience with moving the GBI antennas along a roadway led to an early decision that the VLA antennas would move along two parallel railway lines. Rather than trying to push or pull the antennas using a bulldozer as was done at Green Bank and Caltech, antenna transporters were specially designed to reconfigure the VLA antennas. The initial plan called for three transporters, one working along each arm, but there was only enough money in the budget for two transporters that were christened "Hein's Trein" and "CamTrak" (Fig. 7.8). Also, as a result of the rapid period of inflation following the oil crisis, the cost of used railroad track increased from $\$ 90$ per ton to $\$ 330$ per ton. NRAO hired two retired railway track foremen who were able to locate 14,000 tons of US government surplus track at some 28 different locations around the country. As described by Heeschen (1981), with the aid of the NSF, some of this was declared surplus and made available to NRAO for the cost of shipping to the VLA site. Another 221 tons of new rail was purchased at near scrap prices after it had been rejected by the US Department of

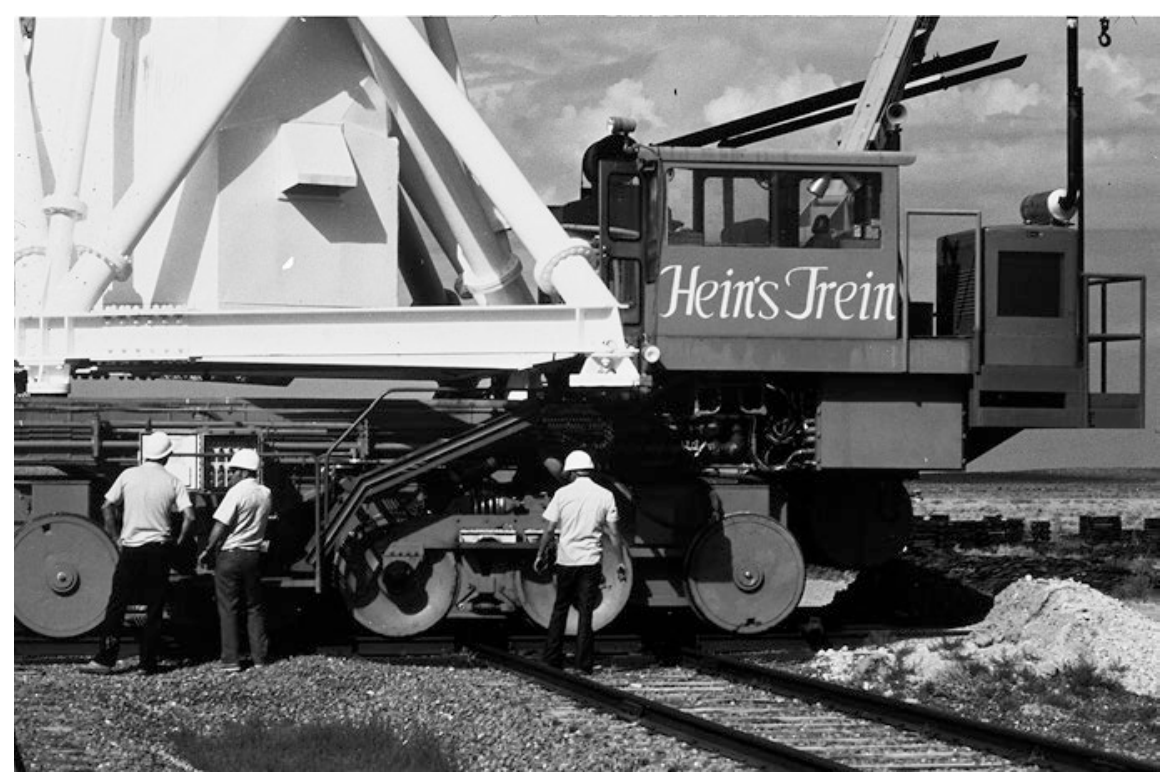

Fig. 7.8 Hein's Trein, used to transport the VLA 25 meter antennas when reconfiguring the array. Credit: NRAO/AUI/NSF 
Transportation for not meeting the required specifications for commercial rail lines (Figs. 7.9 and 7.10).

All of the instrumentation, the front ends, IF systems, digital delays, and the correlator, were designed by NRAO engineers, and for the most part, were fabricated in-house. Other instrumentation, including the monitor and control system, feeds, paramps, cryogenics, and the waveguide distribution system, were fabricated commercially. With a careful system of testing and noting failure rates, redesigns and retrofitting were kept to a minimum. Not unexpectedly, in view of the problems experienced in Green Bank, the cryogenic systems proved to be the least reliable component until a new manufacturer was found.

With the instrumentation of the first completed antenna, and the start of commissioning, the VLA project management, scientists and engineers moved from Charlottesville to temporary headquarters in Socorro, New Mexico, during the spring and summer of 1975. It was a one-hour bus ride, each way, from Socorro to the VLA site. Although on most days most of the staff were not normally needed at the construction site, Lancaster adopted the practice of having everyone-management and administrative personnel, scientists, engineers, and technicians - all ride together to the site on one of two buses. This practice enabled a high level of communication among the disparate groups, which many later agreed was crucial to the successful completion of the VLA.

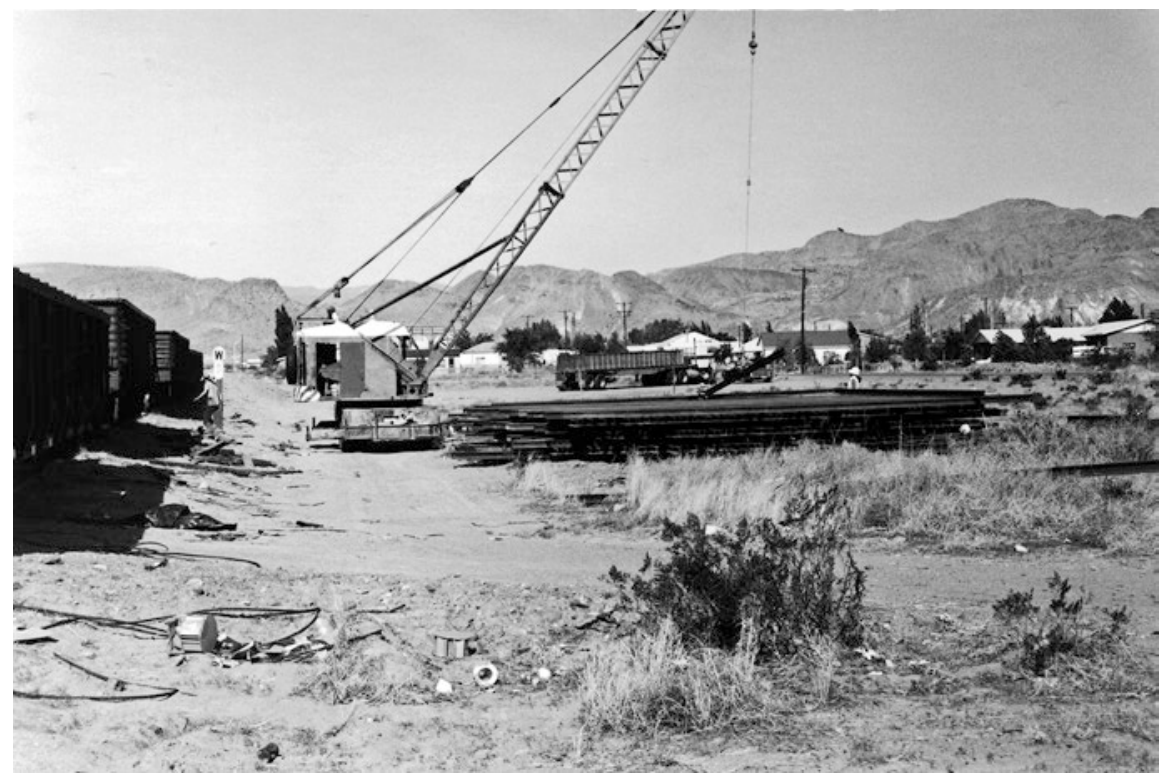

Fig. 7.9 Unloading surplus rail in Socorro. Crane is unloading Crab Orchard rail from rail cars. The truck in the background is leaving with rail for the VLA site. Credit: NRAO/AUI/NSF 


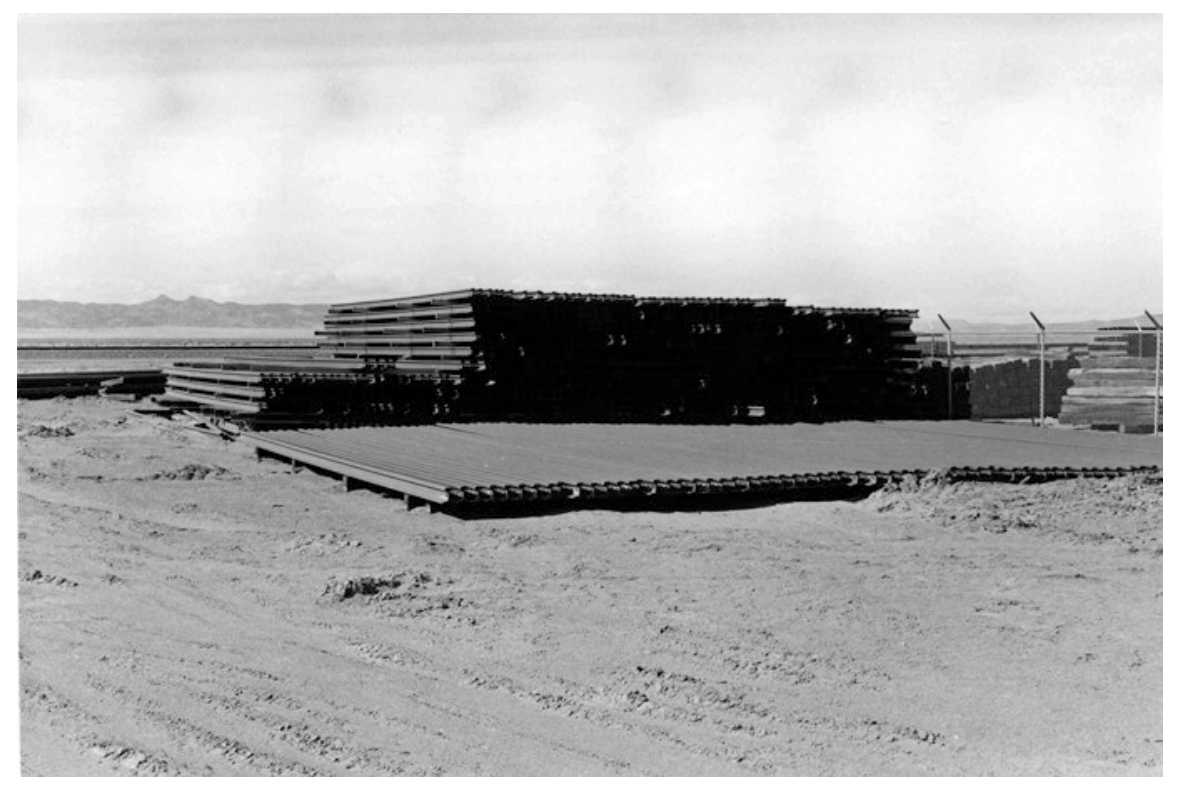

Fig. 7.10 Surplus rail from Holloman Air Force Base arriving at the VLA site. Credit: NRAO/AUI/NSF

The first antenna was completed and moved from the Antenna Assembly Building in July 1975 (Fig. 7.11), less than three years after the initial VLA funding was authorized. First fringes were detected between two antennas over a $1.24 \mathrm{~km}$ baseline in February 1976. By the beginning of 1977 five antennas were in operation using a $2 \mathrm{~km}$ baseline, and the first scientific results from the VLA were reported by Balick et al. (1977). By June 1978, there were ten antennas in operation using a $10.6 \mathrm{~km}$ baseline, and NRAO (1978) announced that the VLA was open for scientific proposals from the community. All 27 antennas were in operation by July 1980 and in use for scientific observations. The installation of all $122 \mathrm{~km}$ of railroad track was completed by the end of 1980. Under the tight management of Heeschen, Lancaster, and Hvatum the VLA was built close to the planned budget and completed on schedule. When completed in January 1981, the VLA met, or, in many cases exceeded, all of its performance goals.

Throughout the eight-year construction, Heeschen continually stressed that NRAO was committed to meet the agreed budget. If some item came in at higher than the planned price, something else had to go. Numerous such adjustments were made during the process; fortunately, many of the deleted items were restored in the later years. As a result of constantly changing budget projections from the NSF, OMB, and Congress, NRAO had prepared nearly 50 different funding schemes by the time the VLA was completed in 1980. The final VLA price tag was $\$ 78.578$ million which was only three percent over 


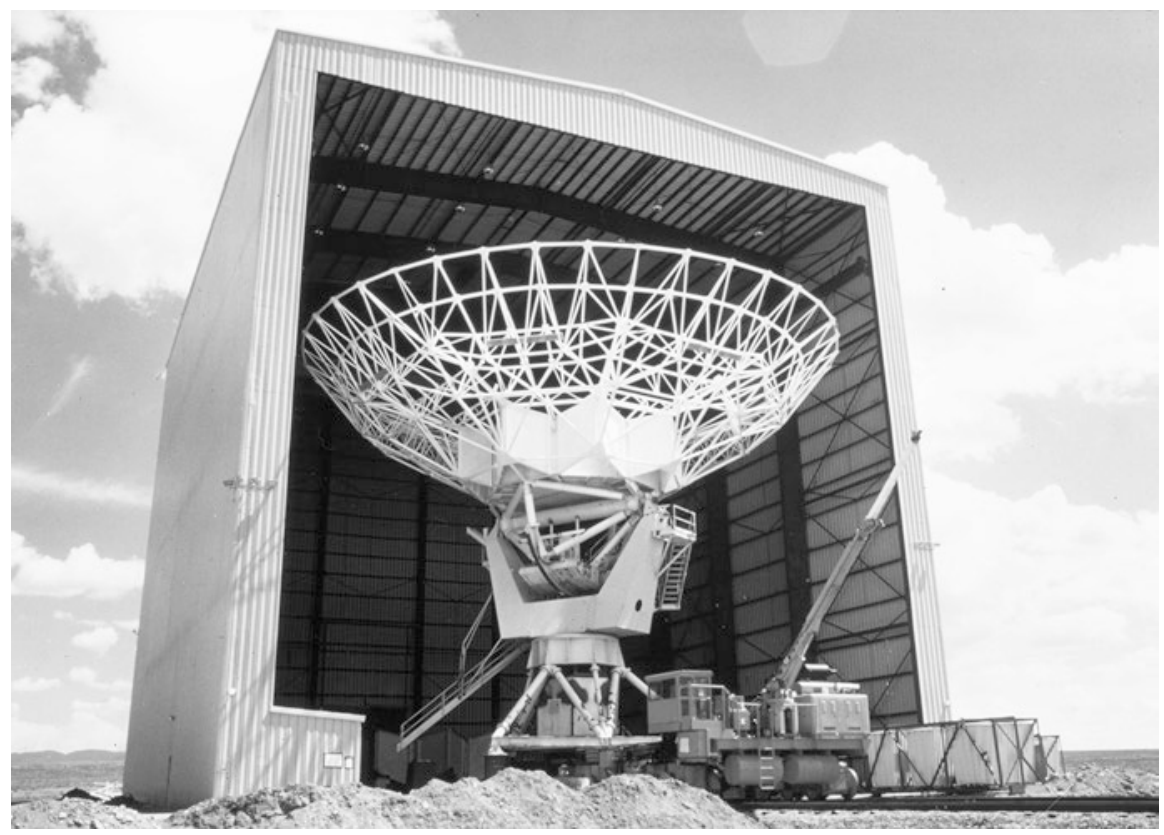

Fig. 7.11 First VLA antenna emerges from Antenna Assembly Building in July 1975. Credit: NRAO/AUI/NSF

the original March 1971 budget of $\$ 76$ million. The increase in the Consumer Price Index over the same period was more than a factor of two. NRAO was able to keep the impact of the unprecedented high inflation modest, due in part to the procurement adjustments made to the fixed-price antenna contract, the more modest rate of inflation for electronic instrumentation, and level or even reduced prices for computing equipment. However, some of the NRAO scientific and administrative staff working on VLA planning and construction remained on the NRAO Operations budget throughout the project, so that the true cost of the VLA was actually somewhat higher than the official number.

Characteristically, Congress and the NSF were nervous throughout the construction period. The VLA was the most expensive NSF project ever attempted and they had to be convinced that it was all going well. In 1976, Congress initiated a review of the VLA project, followed by a Hearing at the House Subcommittee on Science, Research, and Technology. ${ }^{129}$ The subsequent report of the House Science Committee stated, "The Committee is very pleased with the close agreement between the original and current budget and time schedules and commends the project's accomplishments to date." But they worried that the existing NRAO and AUI advisory committees might be concerned with only science and technology and not management, so they added that "The Committee strongly recommends that the Director of the Foundation establish an ad hoc advisory panel to examine the VLA manage- 
ment and technical plans and activities." ${ }^{130}$ Meanwhile, in 1975 and 1976, several delegations of aides from the House Appropriations Committee, including the Chief of Staff, Richard Mallow, ${ }^{131}$ and the House Committee on Science and Technology ${ }^{132}$ visited the VLA. They were mostly concerned about how NRAO was reacting to the potential cost increases resulting from escalation, but also asked about how telescope time would be awarded, how many women were employed on the project, and interestingly, what additions to the array were foreseen for the 1980s. Reportedly, one of the visitors noticed Barry Clark's cluttered office and said, "If this is an example of how the VLA is being run, we're in trouble." 133 Congressional staff also participated in the annual NRAO/AUI presentation to the NSF on 14 February 1976. ${ }^{134}$ This was followed by another hearing held on 30 September 1976 where Dave Heeschen was asked to testify. ${ }^{135}$

The NSF responded to the Congressional mandate by appointing a panel of representatives from industry, universities, and government agencies chaired by Cornell University Vice President Robert Matyas. There were no astronomers on the panel, which met five times during 1977. The panel report issued on 31 December 1977 noted that "The program has now progressed far enough to state with assurance that it will be both a technical and scientific success, ... but also has the potential for discovery in allied fields. ${ }^{" 136}$ However, the panel criticized the NSF for creating difficulties with the stretched-out budget, and recommended that future "projects of this magnitude and complexity be planned and scheduled within a more optimum engineering construction time." The panel praised the project management and the dedication to "living within current budgets and schedules," but raised concerns about the low level of the remaining contingency and the level of effort devoted to software. Interestingly, the panel endorsed the plan "in which a basic publishable 'product' is provided by the VLA facility," a goal which took nearly another forty years to reach. ${ }^{137}$ In addition, the NSF conducted a further audit to "render an opinion as to NSF management on the economy, efficiency and control with which the VLA Project is being administered within the Foundation and by NRAO." Interestingly, eight of the ten recommendations by the audit committee pertained to NSF and not to NRAO record keeping and financial statements. ${ }^{138}$

The official dedication, attended by some 600 staff and guests, was held on 10 October 1980 (Fig. 7.12). Ten years later, at an October 1990 conference sponsored by both the IAU and URSI, more than 220 scientists from 17 different countries gathered in Socorro to celebrate the 10th anniversary of the opening of the VLA. The conference included presentations on astronomy, instrumentation, history, and planning for the future (Cornwell and Perley 1991). The first discussions leading to the Square Kilometre Array began at this meeting (Chap. 11).

In 1973 Heeschen established an internal VLA Steering Committee to replace the defunct VLA Design Group. The Steering Committee met monthly to provide continuing advice on the various aspects of the VLA construction program. Also in 1973, he appointed an external VLA Advisory Committee 


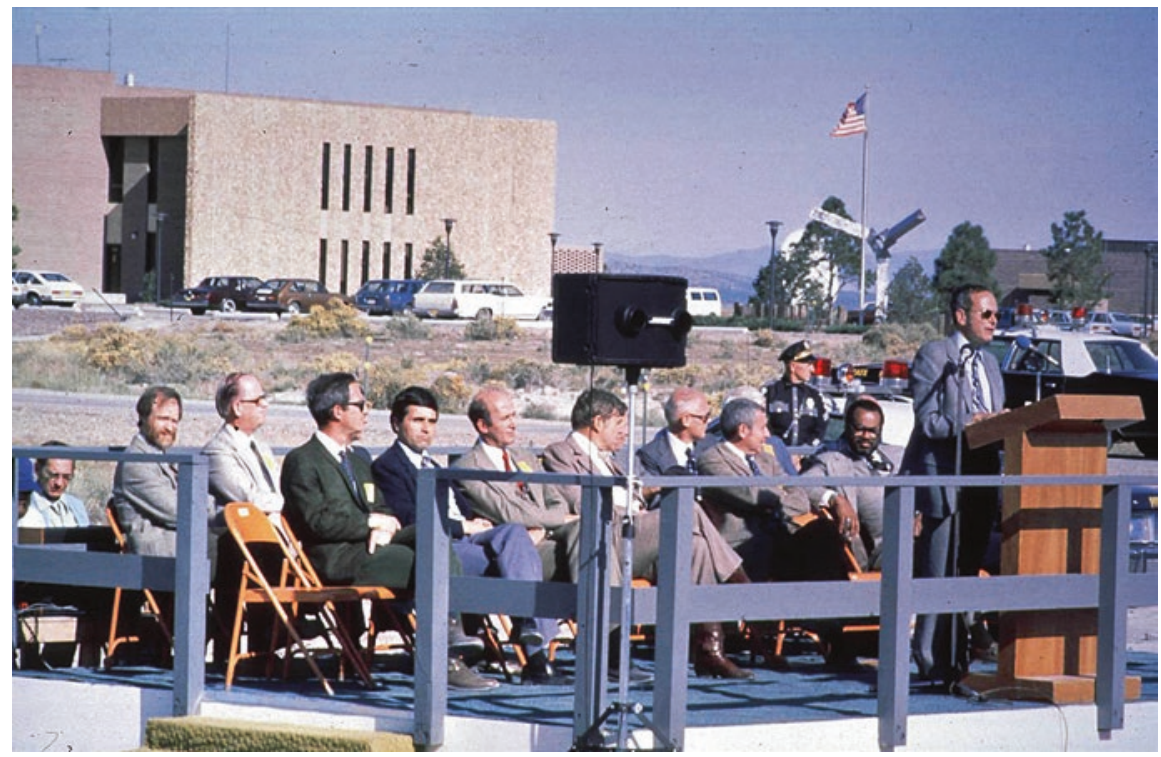

Fig. 7.12 VLA dedication, 10 October 1980; President's Science Advisor Frank Press standing at the podium. Credit: NRAO/AUI/NSF

"to help assure ... that the engineering and construction of the VLA are consistent with the performance goals, and to participate in the further general development of the concept and design of the instrument." 139 The VLA Advisory Committee generally met twice a year throughout the construction project and into the early operations phase. It contributed significantly to setting the final parameters of the VLA as well as addressing various technical problems as they arose. The Committee also drew attention to the lack of sufficient computer power to deal with making images from VLA data.

NRAO's computing difficulties were not confined to the VLA, so in early 1982, Roberts appointed a Computer Advisory Committee to "elicit advice from highly qualified experts in the field." ${ }^{40}$ In appointing the Committee members, Roberts noted the "data explosion in the last half decade," and said, "The recent completion of the Very Large Array (VLA) ... particularly dramatize [sic] this problem." All but one of the members were computing experts from industry and academia, but some of the VLA Advisory Committee members, ${ }^{141}$ such as Burke and Moffet, frequently took part in the meetings, along with relevant NRAO staff.

\subsection{Transition to Operations}

When completed in 1980, the VLA was not only the most powerful radio telescope in the world, it was, not surprisingly, the most complex radio telescope ever built. In recognition of its sophistication and complexity, VLA users 
needed extensive documentation which was initially provided by a widely used user manual known as The Green Book (Hjellming 1978). The Green Book was an indispensable reference source to observing with the VLA and included detailed instructions for post-observation data calibration and imaging. It was ultimately replaced by the Astronomical Image Processing Software (AIPS) and its associated Cook Book.

One of the advantages of array-type radio telescopes is that they are built in steps, and early observations can begin as soon as there is an interesting number of antennas. Also, being able to test many aspects of the final array after only a few antennas were completed and instrumented meant that debugging and the development of observational procedures could begin early. Even the partially completed VLA far exceeded the scientific capability of any other radio telescope, so the user community got a head start on using the VLA.

A key concept of the NRAO plan was, to the extent possible, to transfer project development personnel, especially the scientists and engineers, to operations, and so minimize turnover and exploit the expertise and experience of the development team for operations. When ten antennas became operational in 1978, it was becoming clear that VLA operations needed to be separated from the continuing construction activities, but during the several years of overlap, this meant that operations started more slowly than desired. Richard (Dick) Thompson, who had been the key systems engineer, was placed in charge of the operations phrase. Thompson had begun his career at Jodrell Bank as part of the team that developed radio-linked long baseline interferometry in the late 1950s (Sect. 8.1).

The operation of the VLA, with about 110 employees located in New Mexico, nearly doubled the size of the NRAO staff. The VLA needed a scientist, not an engineer, to coordinate VLA commissioning and operations, and Heeschen asked Cam Wade to serve as the initial Assistant Director for VLA Operations. Dave Heeschen had been the energetic NRAO leader for 18 years, and as the VLA approached completion he decided to step down to return to research and to be able to spend more time with his family. Following a national search, Mort Roberts was named as the NRAO Director, effective 1 October 1978. Roberts and Wade were longtime friends and colleagues, but they had different approaches to management. Things finally came to a breaking point over Wade's supervision of some of the local staff who lived in the small town of Datil about 20 miles west of the VLA site. According to Wade, ${ }^{142}$ who was concerned that the local staff were underpaid, as a small gesture, he and Lancaster let them use an NRAO van to travel between their home and the VLA. When he learned of this practice, Roberts instructed them to stop. Incensed, at what he perceived as micromanagement, Wade responded by resigning as VLA Director, but he agreed to stay on until Roberts could find a replacement. However, in defiance of Charlottesville management, Wade informed one of the technicians that he was to be on 24-hour call, so it would be necessary for him to take the van to Datil each night, and that if anyone else wanted to ride with him that would be OK. ${ }^{143}$ 
Roberts and AUI President Jerry Tape succeeded in recruiting Ronald Ekers to serve as the first permanent director of VLA Operations. Ekers was then a Professor at the University of Groningen in The Netherlands, where he had established himself as an expert in radio interferometry. He had received his $\mathrm{PhD}$ in 1967 working at Parkes under John Bolton and Bart Bok, followed by three years at Caltech and then a year at the Cambridge Institute for Astrophysics with Fred Hoyle. Ekers had been a member of the NRAO VLA Advisory Committee and so was familiar with the VLA. Even a year earlier, he and Heeschen had discussed the possibility of Ekers coming to NRAO as the Director of VLA Operations. However, he had just returned from a year's sabbatical in Australia, and was obliged to return to Groningen, so was unable to accept without negotiating a "buy-out" with the University. Roberts was impatient to remove Wade, and after returning from vacation, Wade found that he had been replaced by Peter Napier as the new VLA Acting Director. NRAO and the University of Groningen finally did work out a deal by which Ekers would spend part of his time in Groningen. Starting in October 1980, Ekers served as the VLA Director of Operations until February 1988, during which time he defined the VLA operation style, much of which continues to this day. ${ }^{144}$ After leaving NRAO, he became the first director of the Australia Telescope National Facility.

NRAO never received the level of funding planned for the effective operation of the VLA. As early as 1973, Ekers drew attention to the apparent inadequacy of the computer plans. ${ }^{145}$ In the early years, this resulted in inadequate computing power to deal with the growing amount of data and the increasingly sophisticated techniques being developed for image processing. Although the power of CLEAN had already been demonstrated by Högbom (2003) in dealing with the poor $u, v$ coverage from the GBI, it wasn't clear if CLEAN would continue to be effective with the initially superior images obtained from the VLA with its better $u, v$ coverage. Many early opponents of the VLA, especially those at Caltech, argued, correctly as it turned out, that the VLA was over-designed to meet the stated goals. However, the use of CLEAN (Högbom 1974, 2003; Schwarz 1978; Clark 1980) and then self-calibration using the closure phase relations (Readhead and Wilkinson 1978; Cotton 1979), resulted in an instrument having very much greater power than originally planned. Moreover, the development of CLEAN, maximum-entropy, and self-calibration enabled the VLA to produce tens or even hundreds of images per day instead of the planned two or three. All of this combined to greatly increase the computing demands. Initially, the available computing power at the site was inadequate to fully exploit these computationally intensive algorithms, and NRAO was criticized for not providing adequate computing power to support the VLA. Heeschen later commented (Tucker and Tucker 1986, p. 31),

The expectations of what people would do with the VLA increased tremendously between the time we first submitted the proposal and the time we eventually began building it. ... The techniques that are used today didn't exist at all in the 
sixties. ... These procedures are extremely valuable, but they increase the dataprocessing requirements by orders of magnitude. When we first designed the computer, we thought it was adequate for what we then thought the thing would do. But, in the interim, so much happened that we simply couldn't afford.

Heeschen resisted the temptation to ask for the additional funds that would have enabled the earlier full exploitation of the VLA, particularly for spectroscopic observations. In January 1980, in order to keep up with the flow of data, use of the VLA was reduced to 50 percent of the available observing time, and full time observing was not restored until April 1981. Adequate computing power was arguably the single biggest constraint to the VLA scientific productivity, and throughout the early 1980s NRAO struggled with the computing issue. The VLA Advisory Committee repeatedly urged NRAO to acquire more computing hardware and devote more resources to VLA software development. The NRAO Computer Advisory Committee recommended a "long range plan based on astronomical requirement ... flexible and growable computer architecture ... [requiring] a major new infusion of capital from the NSF." 146 Fortunately, however, the rapid development of relatively inexpensive powerful work stations and then personal computers, along with adoption of the user-friendly Astronomical Image Processing System (AIPS) (Greisen $1990,1998)^{147}$ and other on-line documentation, ${ }^{148}$ mitigated the computing bottleneck and, ultimately, greatly contributed to the success of the VLA. Combined with the increasing availability of powerful, yet inexpensive work stations, AIPS made it possible for the users to reduce their VLA data at their home institution, thus relieving NRAO of the need to provide a major computing center.

Initially, computing resources were so limited that users were normally restricted to making images no larger than $256 \times 256$ pixels, and needed to specifically request permission if they wanted to make larger images. Although VLA image processing was initially restricted by the limited computing power available, if NRAO had instead chosen what many argued was a more cost effective optical image processing, it would have excluded the use of CLEAN and self-calibration, which would probably have restricted the VLA imaging capability to that proposed in 1967.

As mentioned, with the growing use of inexpensive powerful computers, the data processing was no longer an issue, but with time the VLA hardware became outdated. Other components of the VLA such as the power cables and surplus railroad ties were deteriorating at an alarming rate. Because the A-configuration makes up 70 percent of the VLA's baseline, the high cost of replacing so many railway ties and power cables threatened the continued operation in A-configuration. ${ }^{149}$ Between 1984 and 1986, NRAO added simple uncooled $327 \mathrm{MHz}(92 \mathrm{~cm})$ receivers to the prime focus. But until the 2001 EVLA project (Sect. 7.8), the only major VLA improvement came about from an agreement with NASA to provide observing time on the VLA to download images from the Voyager spacecraft at the time of its encounter with Neptune 
in August 1989. To optimize the VLA sensitivity, NASA provided funds for NRAO to construct state-of-the-art receivers at $8.4 \mathrm{GHz}$, which for a long time remained the most sensitive VLA receiving band. Since support of the Voyager encounter required a higher level of reliability than normal radio astronomy observations, NRAO convinced NASA to provide sufficient funding to also replace the backup power generators, deteriorating railway ties, and power cables supporting the inner configurations.

As discussed in Chap. 8, the decision to co-locate the VLBA Operations Center in Socorro together with the VLA Operations necessitated moving many of the VLA activities to a new Array Operations Center on the New Mexico Tech Socorro campus, which opened in December 1987.

Impact of the VLA The VLA was not only completed on schedule and within budget, but in nearly every respect-sensitivity, resolution, image quality, speed, number of frequency bands, spectroscopic capability, field-of-view-it far exceeded its design goals. As described by Heeschen (1981), the VLA

was motivated by a clearly perceived need, in the early 1960s, for an image forming instrument of the greatest feasible resolution, sensitivity and general versatility. While its designers were most strongly influenced by the opportunities and problems presented by extragalactic radio sources, the need for such an instrument was apparent in all areas of radio astronomy, and the VLA was in fact designed to be used for almost all kinds of radio astronomy studies.

He went on to point out that

The VLA has 10 to 100 times greater resolution and sensitivity than any other existing radio telescope, and its resolution is comparable to or greater than that obtainable at optical and other wavelengths. Its speed, sky cover, ability to measure polarization, and ability to make high frequency, high resolution spectroscopic observations give it tremendous power and versatility for a wide variety of problems.

Perhaps the biggest change brought about by the VLA was that, prior to the VLA, radio astronomy was pretty much a "black belt" experience confined to those with training or experience in radio astronomy. NRAO users were expected to do their own calibration and analysis. Student use generally required attentive support from a faculty or in some cases an NRAO staff advisor. The power and complexity of the VLA led NRAO to provide more handson support for users, which, in turn, began to attract a broader group of astronomers who needed radio data to enhance their research program. ${ }^{150}$

To support the growing user community, and especially to train the new generation of scientists who would use the VLA, NRAO started the very successful series of synthesis imaging workshops. The first workshop held in Socorro in June 1982 was attended by 85 scientists and students (Thompson 
and D'Addario 1982) and was followed by similar workshops held every two or three years.

An unanticipated use of the VLA developed when NRAO received two separate proposals to use the VLA for sky surveys intended to detect and catalogue an unprecedented number of discrete radio sources. One proposal was from an internal group led by Jim Condon, who proposed an "all-sky" survey with a resolution of the order of an arcmin to detect the nearly two million radio sources stronger than $2.5 \mathrm{mJy}$. A competing proposal came from a group led by Robert Becker from the University of California, Davis, proposing a deeper, higher resolution survey that would reach sources as faint as $1 \mathrm{mJy}$, but only covered the limited area of the sky corresponding to the Sloan Digital Sky Survey (SDSS) (York et al. 2000). With the higher angular resolution, the proposed Becker survey would give more accurate source positions needed for optical identification with SDSS counterparts, as well as imaging the arcsec structure of detected radio sources. However, unlike the NRAO survey, it would be insensitive to larger scale radio emission. The two proposed surveys were not only in competition, but each wanted thousands of hours observing time. The NRAO Director, Paul Vanden Bout, polled the user community, who were mostly supportive of the two proposals, so he appointed a small internal committee chaired by Frazer Owen to make a recommendation on choosing which proposal to approve. Recognizing the complementarity of the two projects, Owen's committee recommended that they both be approved. But once the observing began, other users, including those who supported the idea of big projects, complained that they were taking up too much observing time.

The two projects were each completed, but to minimize the impact to other observers, each was stretched out over a number of years. Both the Condon et al. (1998) NRAO VLA Sky Survey (NVSS) and the Becker et al. (1995) Faint Images of the Radio Sky at Twenty Centimeter (FIRST) were enormously successful resources for the astronomy community, each receiving thousands of citations. To help resolve any future quandaries between the merits of large proposals and their impact to other programs, Vanden Bout convened a "Large Proposal Review Committee" chaired by NRAO staff member Alan Bridle.

The VLA has also helped to propel radio astronomy into the popular media, appearing in several movies and numerous TV ads. The popular movie Contact brought particular attention to the VLA, although it gave a misleading impression that the VLA is used to search for signals from extraterrestrial intelligent civilizations. Images of the VLA appear frequently in TV advertisements, although mostly for products and services unrelated to radio astronomy.

The impact of the VLA was not all positive. Faced with limited operating budgets, the NSF could not support the expensive VLA operations at the same time as the many more modest university-operated facilities. Funding was cut for the Owens Valley Observatory interferometer, where many of the techniques used at the VLA had been developed and where many of NRAO staff 
that built the VLA were trained. Only modest support remained for operating the OVRO 130 foot antennas as an element of the US VLBI Network (Chap. 8). The five-element interferometer built by Ron Bracewell at Stanford University with NSF funds was closed soon after it was completed. A novel low frequency array built by Bill Erickson in the Southern California desert lost most of its NSF support. American radio astronomers and their students were more and more driven to the national observatory facilities operated by NRAO and NAIC. The reduction of the once vibrant university radio astronomy groups restricted the training of the next generation of technically-skilled observers, further increasing the pressure on NRAO to provide a turn-key observing opportunity at all of its facilities.

\subsection{The Karl G. Jansky Very Large Array (JVLA)}

The VLA has arguably been the most productive ground-based telescope ever built, certainly the most productive radio telescope (Trimble and Ceja 2008), and has more than lived up to Dave Heeschen and NRAO's expectations. Although conceived primarily for extragalactic continuum research, the VLA has been used to study essentially all fields of astronomy from the Sun and Solar System bodies to a wide range of galactic and extragalactic phenomena. A particular surprise has been the large fraction of time spent studying the radio emission from stars, an area not even mentioned in the 1965 VLA Report No. 1. Spectroscopic studies, which were mentioned only in passing in the 1967 proposal, have typically represented about one-third of the VLA observing time. As noted by Trimble and Zaich (2006),

The VLA [is] responsible for $22 \%$ of the papers and 27 percent of the citations [in radio astronomy]. The VLA is, therefore, proportionally even more influential in world radio astronomy than HST is in world optical astronomy.

When built in the 1970s, the VLA receivers, waveguide transmission, and digital correlator were all state-of-the-art. However, the limited funding made available for VLA operations, combined with the rapid advances in technology, meant that only two decades after its dedication the VLA instrumentation was becoming woefully obsolete. Moreover, the great changes in astronomy over the last few decades of the twentieth century led to new demands for better sensitivity and image quality as well as improved spectral and angular resolution. ${ }^{151}$ Responding to these forces, in May 2000 NRAO/AUI submitted a proposal to the NSF to increase the sensitivity of the VLA by up to an order of magnitude, provide better frequency coverage, and greatly improved spectroscopic capability. ${ }^{152}$ NRAO intended this to be just the first phase of the VLA upgrade, but the Phase II proposal ${ }^{153}$ to "expand" the VLA by adding additional antennas, was never funded, although the name "Expanded Very Large Array" (EVLA) stuck, at least throughout the construction period. Following review by the NSF, the EVLA Phase I project was partially funded through an 
increment to the NRAO operating budget rather than through the Major Research Equipment and Facilities Construction (MREFC) budget, but about half of the $\$ 96$ million cost of the EVLA was born by the base VLA operating budget. Additional funding came from in-kind contributions from the Consejo Nacional de Ciencia y Technologia (CONACyT) ${ }^{154}$ of Mexico, and from the Canadian National Research Council for the WIDAR (Wide-band Interferometer Digital ARchitecture) correlator. The Memorandum of Understanding (MOU) with Canada was far reaching and established the North American Program in Radio Astronomy (NAPRA) which, in return for the Canadian WIDAR correlator, gave Canadian scientists the same access as US scientists to all NRAO facilities. The NAPRA agreement included Canadian participation in ALMA as well as joint NRAO-Canadian efforts toward the SKA development. ${ }^{155}$

Upgrading the VLA instrumentation began in 2001 and was completed a decade later. A particularly challenging aspect was the stated goal of keeping the VLA in operation throughout the decade-long EVLA construction period, which meant simultaneously operating combinations of old and upgraded antennas with first the old and then the new correlator. Except for a sevenweek period in 2010 when the old VLA correlator was replaced by the new one, the VLA remained in operation during the entire 10 years of construction. The upgraded VLA, which was dedicated on 31 March 2012, was given a new name, the Karl G. Jansky Very Large Array (JVLA), replacing the term "EVLA," which has since been redefined to refer to the construction project and not the instrument (Fig. 7.13).

The JVLA has complete frequency coverage across the entire band from 1 to $50 \mathrm{GHz}$ provided by eight feeds and receivers at the secondary focus. A ninth receiving band at the prime focus covers the range from 50 to $450 \mathrm{MHz}$ with reduced sensitivity. The front end systems are based on cooled HEMT amplifiers and corrugated feed horns, while the local oscillator is derived from a hydrogen maser frequency standard, and distributed via fiber to each of the 27 antennas. The IF signals are digitized at the antenna and sent by fiber to the new WIDAR correlator, designed by Brent Carlson from Canada. The WIDAR combines the data on each of the 351 interferometer baselines at up to $10^{15} 32$ bit operations per second to give up to four million frequency channels, or, for continuum studies, up to $8 \mathrm{GHz}$ total bandwidth in each of two polarizations. More detailed descriptions of the JVLA are given by Perley et al. $(2009,2011)$. The basic performance parameters of the JVLA are shown in Table 7.1 compared with the 1967 proposal, what was achieved in 1980 at the end of VLA construction, and in 1986 after various hardware and software upgrades.

The JVLA reaches unprecedented levels of sensitivity extending to below 1 $\mu \mathrm{Jy}$ in a 12 hour integration. But with the improved sensitivity and larger bandwidths came the need for better interference suppression and improved dynamic range in order to reach the thermal sensitivity limits of the array in long integrations. New imaging algorithms and new software needed to meet these challenges was implemented both within AIPS (Greisen 1990) as well as 


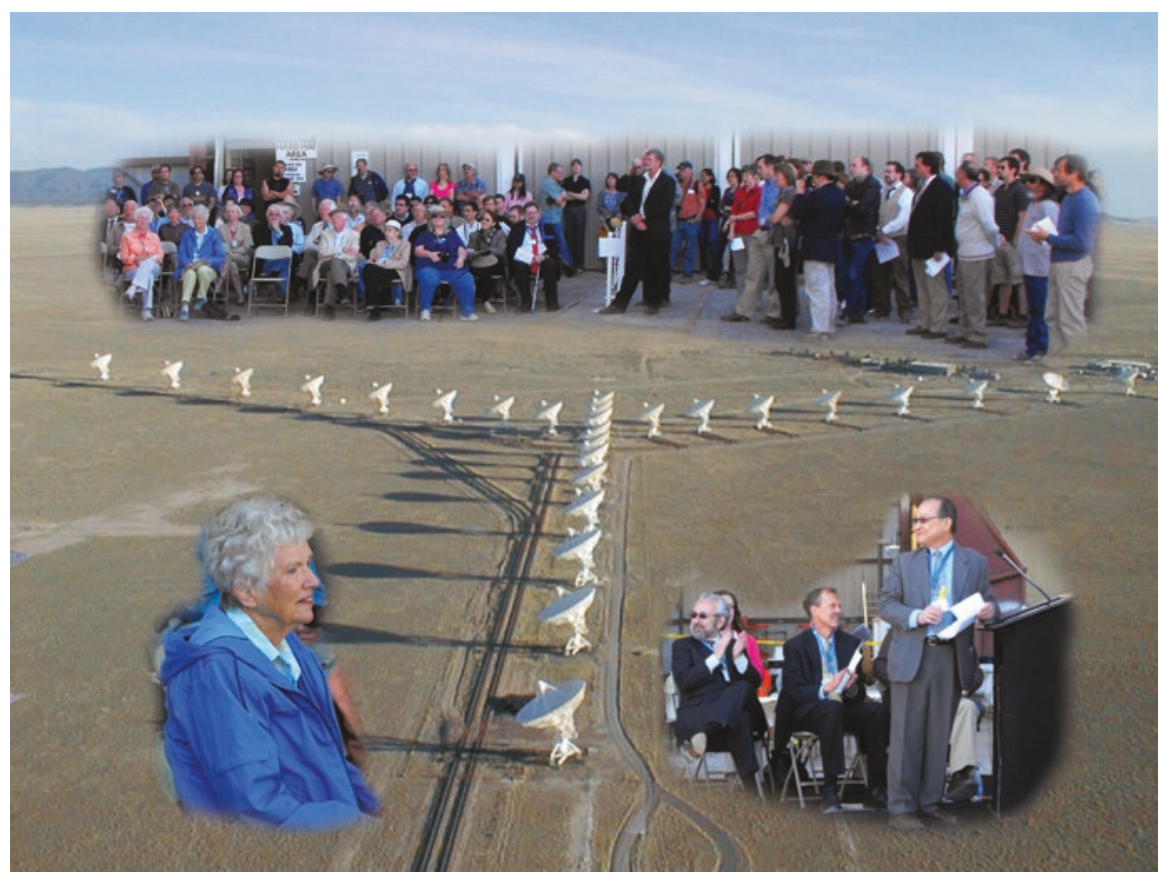

Fig. 7.13 Karl G. Jansky Very Large Array rededication, 31 March 2012. Center: The JVLA in D array. Top: Attendees at the rededication ceremony. Lower right: NRAO Director Fred Lo (standing) initiates the start of first official Jansky VLA observation. Seated behind Lo are Ethan Schreier, AUI President, and James Ulvestad, NSF Astronomical Sciences Division Director. Lower left: Anne Moreau Jansky Parsons, daughter of Karl Jansky. Credit: D. Fiinley/NRAO/AUI/NSF

Table 7.1 VLA performance

\begin{tabular}{lllll}
\hline Parameter & 1967 & 1980 & 2000 & 2011 JVLA \\
\hline Resolution (arcsec) & 1 & 0.1 & 0.04 & 0.04 \\
Sensitivity ( $\mu \mathrm{Jy})$ & 300 & 50 & 10 & 1.5 \\
Dynamic Range & 100 & 1000 & 100,000 & 200,000 \\
Field of View (arcmin) & 1 to 10 & 1 to 30 & 1 to 300 & 1 to 300 \\
No. $\lambda$ Bands & 2 & 4 & 6 & $1-40 \mathrm{GHz}$ \\
& & 1.3 & 1.3 & continuous \\
Shortest Wavelength (cm) & 11 & $-40^{\circ}$ to $+90^{\circ}$ & $-40^{\circ}$ to $+90^{\circ}$ & $-40^{\circ}$ to $+90^{\circ}$ \\
Declination Range & $-20^{\circ}$ to $+90^{\circ}$ & 100 & 200 & 20,000 \\
Speed (images/day) & 3 & 256 & 512 & $4 \times 10^{6}$ \\
No. Freq. channels & none & $512 \times 512$ & $4096 \times 4096$ & $4096 \times 4096$ \\
Map Size (pixels) & $100 \times 100$ & & &
\end{tabular}

Notes: The first three columns are adopted from Ekers' 1987 report on the First Seven Years of VLA Operations (Ekers 1987, op. cit.) and the final column from Perley et al. (2011). The values given for the sensitivity represent the $3 \sigma$ detection level from a 12 hour synthesis image. The specification of the dynamic range is a bit subjective as it depends in part on the complexity of the field being imaged. The number of images per day given for the JVLA is based on the number of independent fields observed in the NRAO VLA Sky Survey using a scan rate of $3.31 \mathrm{arcmin} / \mathrm{sec}$ 
CASA (Common Astronomical Software Application) (McMullin et al. 2007). Although data analysis, especially from the first few years of the JVLA, was rather labor intensive, the publication of a special issue of the Astrophysical Journal Letters in 2011 (Vol. 739), demonstrated the already wide range of possible research and the enormous potential of the JVLA for new discoveries. ${ }^{156}$

\section{Notes}

1. Detailed reviews of synthesis imaging in radio astronomy are given by Taylor et al. (1999), Kellermann and Moran (2001), Thompson et al. (2017), and in references contained therein.

2. The recent implementation of adaptive optics has enabled some ground based telescopes to achieve diffraction limited imaging at near infrared wavelengths.

3. The so-called "sea interferometer" combined the signal received from reflection by the ocean with the directly received signal to form a two element interferometer, although only one antenna was used.

4. The authors do not elaborate, but presumably they are talking about a conventional 2-element interferometer. Apparently Pawsey had been the first author on this paper, but the author list was rearranged to be alphabetical.

5. Earlier, as part of Cambridge PhD work, Patrick O'Brien and Peter Scheuer (Scheuer 1984) and Högbom $(1959,2003)$ used Earth rotation synthesis to image the Sun in 1953 and 1958 respectively.

6. Swenson, G.W. Jr. 1977, A Case Study of the Decision to Construct a Large Radio Telescope, (Washington: National Academy of Sciences Joint Working Group on National Systems for the Stimulation of Fundamental Research), NAA-NRAO, NM Operations, VLA Design and Construction.

7. The design and construction of the Westerbork Synthesis Radio Telescope, including the earlier considerations of the Benelux Cross, are discussed by Raimond (1996).

8. Charles Seeger was the brother of the folk singer Pete Seeger.

9. In all radio arrays, data corresponding to interferometer spacings comparable to and smaller than the antenna diameter is missing and this results in the inability to observe structures whose angular scale is comparable with the primary beam of the individual elements.

10. Each of the redundant spacings from different antenna pairs sampled the same Fourier component of the sky, so any differences in the measured interferometer response were due to instrumental, tropospheric, or ionospheric effects.

11. Detailed technical descriptions of the WSRT are given by Baars et al. (1973), by Baars and Hooghoudt (1974), and by Högbom and Brouw (1974).

12. The other members of the panel included Ronald Bracewell (Stanford), Bernard Burke (Carnegie Institution of Washington), P. Chena (Purdue), David Heeschen (NRAO), Rudolph Minkowski (University of California Berkeley), L.J. Chu (MIT), Richard Emberson (AUI), William Gordon (Cornell), George Swenson (University of Illinois), and J.H. Trexler (Naval Research Laboratory). John Bolton, representing Caltech, served as a consultant to the Panel, but returned to Australia before the work of the Panel was 
completed. NSF Astronomy Director Geoffrey Keller (1961) served as liaison between the Panel and the NSF and authored the report.

13. RH interview with CMW, 29 December 2003. https://science.nrao.edu/ about/publications/open-skies\#section-7

14. DSH Memo to Findlay, Drake, Hvatum, Wade, Hogg, Vinokur, Callendar, 5 March 1962, NAA-NRAO, Founding and Organization, Antenna Planning. https://science.nrao.edu/about/publications/open-skies\#section-7

15. Ibid.

16. Ibid.

17. J. Pawsey, Notes of Future Program at Green Bank, 17 July 1962, NAANRAO, Founding and Organization, Antenna Planning. https://science. nrao.edu/about/publications/open-skies\#section-7

18. Pawsey sent copies of his letter to Heeschen, Tape, and Rabi, with a note to Heeschen that "it is highly provisional and could well be modified after further discussion with you people in Green Bank." Pawsey to Erickson, 27 June 1962, NAA-NRAO, Founding and Organization, Antenna Planning. https:// science.nrao.edu/about/publications/open-skies\#section-7

19. Pawsey to Scheuer, 4 July 1962, NAA-NRAO, Founding and Organization, Antenna Planning. https://science.nrao.edu/about/publications/open-skies \#section-7

20. Erickson to Pawsey, 28 August 1962, NAA-NRAO, Founding and Organization, Antenna Planning. https://science.nrao.edu/about/publications/open-skies\#section-7

21. DSH to J. Findlay, Ivan Pauliny-Toth, M. Vinokur, C. Wade, and V. Venugopal, 7 September 1962, NAA-NRAO, NM Operations, VLA, Design and Construction, Box 1.

22. AUI-BOT, 18 January 1963.

23. Request for a Proposal for Antenna Design Study, 8 November 1962, NAANRAO, Founding \& Organization Series, Antenna Planning.

24. KIK interview with CMW, 21 March 2015, NAA-KIK, Oral Interviews. The VLA did not use fiber optic links until the EVLA upgrade 40 years later. https://science.nrao.edu/about/publications/open-skies\#section-7

25. CMW, November 1963, NAA-NRAO NM Operations, VLA, Design and Construction. https://science.nrao.edu/about/publications/open-skies\# section-7

26. $\mathrm{RH}$ interview with $\mathrm{CMW}$, op. cit.

27. KIK interview with DSH, 31 May 2011, NAA-KIK, Oral Interviews. https:// science.nrao.edu/about/publications/open-skies\#section-7

28. AUI-BOTXC, 15 February 1963.

29. AUI-BOTXC, 18 February 1966.

30. Caltech Proposal to the NSF, 1962, NAA-KIK, Open Skies.

31. Ironically, a few years later the Caltech 90 foot dishes were converted to alt-az mounts.

32. Vinokur to DSH and JWF, 30 October 1962, NAA-NRAO, Founding and Organization, Antenna Planning.

33. Other committee members included radio astronomers Ronald Bracewell, Frank Drake, and Fred Haddock along with optical astronomers William Liller, W.W. Morgan, and Allan Sandage. Whitford, A. 1964, Ground-based 
Astronomy: A Ten Year Program, National Academy of Sciences, Washington, hereafter referred to as the Whitford Committee.

34. Summary Minutes of the Ad Hoc Meeting on Radio Astronomy, November 1 and 2, 1963, ANAS, Central File, COSPUP, Astronomical Facilities.

35. Ibid.

36. Ibid.

37. During this period the Consumer Price Index increased by a factor of 2.4 .

38. AUI-BOTXC, 16 September 1965.

39. The main contributors to the design report were L.C. Chow, B.G. Clark, D.S. Heeschen, D.E. Hogg, H. Hvatum, G.W. Swenson, W.C. Tyler, and C.M. Wade. From time to time, a few external people, including Frank Drake and Bernie Burke, participated in the monthly design meetings.

40. AUI-BOT, 20 January 1966.

41. AUI-BOTXC, 17 August 1967.

42. CMW to DSH and JWF, 3 November 1962, NAA-NRAO, Founding and Organization, Antenna Planning.

43. AUI-BOT, 18 January 1963.

44. AUI-BOTXC, 16-17 July 1964.

45. NRAO, November 1969, GBI Memo No. 25, The NRAO Interferometer, A Development Program. http://library.nrao.edu/public/memos/gbi/ GBI_025.pdf

46. CMW, November 1964, GBI Memo No. 4, A Method for Finding the Phase and Amplitude of Interferometer Fringe Patterns. http://library.nrao.edu/ public/memos/gbi/GBI_004.pdf

47. BGC, December 1964, GBI Memo No. 7, On the Reduction of Digital Interferometer Records. http://library.nrao.edu/public/memos/gbi/ GBI_007.pdf

48. Clark, B.G. and Wade, C.M., April 1965, GBI Memo No. 14, The Interferometer Fringe Reduction Program. http://library.nrao.edu/public/ memos/gbi/GBI_014.pdf

49. AUI-BOT, 20 January 1966.

50. BGC, July 1968, GBI Memo No. 23, Programs for the DDP-116 Computer. http://library.nrao.edu/public/memos/gbi/GBI_023.pdf

51. NRAO, November 1969, The NRAO Interferometer, A Development Program, op. cit.

52. Caltech Proposal to the NSF, April 1966, NAA-KIK, Open Skies.

53. CITA-GJS, unprocessed papers.

54. Ibid.

55. Other committee members were Bart Bok (University of Arizona), Stirling Colgate (New Mexico Institute of Mining and Technology), Rudolph Kompfner (Bell Laboratories), William Morgan (Yerkes Observatory), Eugene Parker (University of Chicago), Merle Tuve (Carnegie Institution Department of Terrestrial Magnetism).

56. https://science.nrao.edu/about/publications/open-skies\#section-7

57. KIK interview with CMW, 21 March 2015, op. cit.

58. The Owens Valley Array - 1969, A Report to the Second Session of the Dicke Committee on Facilities for Radio Astronomy, NAA-KIK, Open Skies.

59. AUI-BOT, 18 January 1968. 
60. KIK interview with CMW, 21 March 2015, op. cit.

61. The Panel membership was retained from the first meeting, although Merle Tuve was unable to attend the second meeting of the Panel. https://science. nrao.edu/about/publications/open-skies\#section-7

62. GJS to Anderson, R. Bacher, and L. Bonner, 20 November 1968a, CITA-GJS.

63. GJS to Anderson, R. Bacher, and L. Bonner, 20 November 1968b, CITA-GJS.

64. GJS to R. Bacher, 3 April 1969, CITA-GJS.

65. KIK interview with DEH, 26 March 2013, NAA-KIK, Oral Interviews.

66. Tape to J. Bergen, 12 January 1970, NAA-NRAO, New Mexico Operations, VLA, Design and Construction.

67. J. Bergen to Tape, 17 February 1970, NAA-NRAO, New Mexico Operations, VLA, Design and Construction.

68. Bureau of the Budget (BOB), Interagency Continuing Special Study: Federally Supported Astronomy, undated. NAS-NRC-A, Central File: Divisions of the NRC: Physical Sciences: Astronomy Survey Committee: 1970. The BOB prospectus contained six major astronomy projects, two of which, "a large radio astronomy array" and "a large steerable antenna," were for radio astronomy.

69. L. Haworth to H. Brooks, 22 January 1969, NAS-NRC-A, Central File, ADM: Committees and Boards: COSPUP Panels: Astronomical Facilities: Report: General: 1965. Brooks was the Chairman of the NAS Committee on Science and Public Policy (COSPUP).

70. Proposal for Support of the Study of Priorities in Astronomy June 1, 1969September 30, 1971, NAS-NRC-A, Central File, ADM: Committees and Boards: COSPUP Panels: Astronomical Facilities: Report: General: 1965. The Committee on Science and Public Policy (COSPUP) was renamed in 1982 as the Committee on Science, Engineering, and Public Policy (COSEPUP), and in 2016 as the Committee on Science, Engineering, Medicine, and Public Policy (COSEMPUP).

71. Haworth to Brooks, 27 May 1969, NAS-NRC-A, Central File, ADM: Committees and Boards: COSPUP Panels: Astronomical Facilities: Report: General: 1965.

72. Greenstein has related his role in early radio astronomy in an unpublished memoir, I Was There in the Early Years of Radio Astronomy, NAA-KIK, Open Skies.

73. Greenstein to Brooks, 7 July 1969, NAS-NRC-A, Central File, ADM: Committees and Boards: COSPUP Panels: Astronomical Facilities: Report: General: 1965.

74. The other members of the radio panel were Geoffrey Burbidge (University of California San Diego), George Field (University of California Berkeley), Gordon Pettengill (MIT), James Warwick (University of Colorado), and Gart Westerhout (University of Maryland). All were radio astronomers except Field and Burbidge, who were theoreticians, although Field had had some experience as a radio observer at NRAO. Burbidge was also a member of the joint Astronomy/Physics Panel on Relativity and Astrophysics.

75. Greenstein to Radio Panel, 14 October 1969, NAA-NRAO, Director's Office, Professional Organizations/Committees, NAS Committees. 
76. Minutes of the Radio Panel Meeting, 14 November 1969, NAA-NRAO, Director's Office, Professional Organizations/Committees, NAS Committees.

77. Report of the Radio Astronomy Panel of the Astronomy Survey Committee, 1 December 1969, NAA-NRAO, Director's Office, Professional Organizations/ Committees, NAS Committees.

78. Handler to McElroy, 16 December 1969, NAS-NRC-A, Central File: Divisions of the NRC: Physical Sciences: Astronomy Survey Committee: 1970.

79. President Nixon had appointed Caltech President Lee DuBridge as his Science Advisor and Chair of the Presidents Science Advisory Committee (PSAC).

80. Handler to JLG, 16 December 1969, NAS-NRC-A, Central File: Divisions of the NRC: Physical Sciences: Astronomy Survey Committee: 1970.

81. JLG to Handler, 22 December 1969, NAS-NRC-A, Central File: Divisions of the NRC: Physical Sciences: Astronomy Survey Committee: 1970.

82. DSH to JLG, 10 March 1970, NAA-NRAO, Director's Office, Professional Organizations/Committees, NAS Committees.

83. Handler to JLG, 20 November 1969, NAS-NRC-A, Central File: Divisions of the NRC: Physical Sciences: Astronomy Survey Committee: 1970.

84. JLG to DSH, 31 March 1970, NAA-NRAO, Director's Office, Professional Organizations/Committees, NAS Committees.

85. JLG to Handler, 1 June 1970, NAS-NRC-A, Central File: Divisions of the NRC: Physical Sciences: Astronomy Survey Committee: 1970.

86. DSH to JLG, 23 April 1970, NAA-NRAO, Director's Office, Professional Organizations/Committees, NAS Committees, Astronomy Study; CITAJLG, Box 96, Folder 12.

87. Brooks to JLG, 16 March 1970, NAA-NRAO, Director's Office, Professional Organizations/Committees, NAS Committees.

88. Minutes of the Third Meeting of the Radio Panel, 9-10 April 1970, NAANRAO, Director's Office, Professional Organizations/Committees, NAS Committees.

89. They were Barry Clark, Ed Fomalont, and one of the present authors (KIK). MHC to DSH, 2 October 1970, CITA-GJS.

90. Brooks to Handler, 3 September 1970, NAS-NRC-A, Central File: Divisions of the NRC: Physical Sciences: Astronomy Survey Committee: 1970.

91. Handler to Brooks, Dicke, Greenstein, and Whitford, 15 December 1970, NAS-NRC-A, Central File: Divisions of the NRC: Physical Sciences: Astronomy Survey Committee: 1970.

92. McElroy to Handler, 30 October 1970, NAA-NRAO, Director's Office, Professional Organizations/Committees, NAS Committees; NAS-NRC-A, Central File: Divisions of the NRC: Physical Sciences: Astronomy Survey Committee: 1970.

93. Brooks to Handler, 21 December 1970, NAS-NRC-A, Central File: Divisions of the NRC: Physical Sciences: Astronomy Survey Committee: 1970.

94. In 1970, President Nixon replaced the Bureau of the Budget (BoB) with the Office of Management and Budget (OMB).

95. Brooks to Handler, 21 December 1970, op. cit.

96. Minutes of the NSB, 2-4 September 1970, NSB-70-265.

97. Greenstein was an exception. In 1969 at a meeting of the National Research Council Division of Physical Sciences, Greenstein emphasized the need for building bigger optical telescopes to address the big problems. 
98. KIK Interview with DSH, 31 May 2011, op. cit.

99. JLG, unpublished memoir, I Was There in the Early Years of Radio Astronomy, op. cit.

100. Ibid.

101. KIK interview with JLG, January 1995.

102. Cohen to DSH, 23 April 1971, NAA-NRAO, Director's Office, Professional Organizations/Committees, NAS Committees.

103. David to McElroy, 12 April 1971, NAA-NRAO, Director's Office, Professional Organizations/Committees, NAS Committees.

104. KIK interview with BFB, 20 June 2012, NAA-KIK, Oral Interviews. https:// science.nrao.edu/about/publications/open-skies\#section-7

105. GJS to H. Brown, R. Bacher, C. Anderson, JLG, 11 May 1970, CITA-GJS.

106. GJS, et al. 1969, The Owens Valley Array - 1969; A Report to the Second Session of the Dicke Committee on Facilities for Radio Astronomy, NAA-KIK, Open Skies.

107. RH interview with CMW, op. cit.

108. CMW to KIK, 22 June 2018 telephone interview.

109. Limbaugh Engineers, Inc. studied two sites in New Mexico, two in Nevada, and one each in Arizona, Texas, and Utah. They submitted an interim report in August 1966 and a more detailed final report in November 1966.

110. RH interview with CMW, op. cit.

111. DSH to T. Owen, 7 February 1972, NAA-NRAO, NM Operations, VLA Site Selection, Procurement, and Development.

112. Very Large Array (VLA) Site Selection Review Panel: NAS-NRC-A, Central File: Divisions of the NRC: Physical Sciences: 1972.

113. The circular waveguide used the so-called $\mathrm{TE}_{01}$ transmission mode which has very low loss, but only if bends are avoided.

114. NRAO VLA Electronics Memos No. 105, 120, 128, 145, 146, 150-155, 179. http://library.nrao.edu/vlae.shtml

115. To maintain coherence, the signals being correlated need to be synchronized to an accuracy of the order of $1 /$ bandwidth.

116. The VLA correlator allowed various combinations of frequency resolution and bandwidth ranging from 16 channels covering $50 \mathrm{MHz}$ bandwidth $(3.125$ frequency resolution) to 256 channels covering $97 \mathrm{kHz}$ (381 Hz resolution).

117. VLA Electronics Memo No. 207, The Correlator System for the Very Large Array, C. Broadwell and R. Escoffier, July 1982. http://library.nrao.edu/ VLAE_207.shtml

118. See NRAO VLA Computing Memo No.122. http://library.nrao.edu/pub$\mathrm{lic} / \mathrm{memos} / \mathrm{vla} / \mathrm{comp} /$ VLAC_122.pdf

119. An initial software effort called CANDID led by Bob Hjellming was an almost complete failure. Sadly, Bob Hjellming died in 2000 after suffering a heart attack during a diving training class.

120. R. Duquet, NRAO VLA Computer Memorandum 172. http://library.nrao. edu/public/memos/vla/comp/VLAC_172.pdf. For more detailed information on the history of the implementation of the VLA computing hardware and software systems see the series of VLA computing memoranda at http:// library.nrao.edu/vlac.shtml

121. RH interview with CMW, op. cit. 
122. The tortuous path of the VLA funding bill through Congress was described in the The Money Maze: How a Group of People Wanting a Big Telescope Fared in Washington, Wall Street Journal, I November 1973, and by Gloria Lubkin (1975).

123. Ibid.

124. Ibid.

125. Stanford Research Institute, VLA Feasibility Study Final Report, February 1972 (pts. 1-2) and March 1972 (pt. 3), NRAO-NAA, New Mexico Operations, VLA, Design and Construction.

126. This section is based in part on the detailed report by Jack Lancaster (1982) on construction of the VLA, NAA-NRAO, NM Operations, VLA Operations. https://science.nrao.edu/about/publications/open-skies\#section-7

127. NRAO RFP-VLA-01 Antenna Procurement Contract Selection Committee Final Report, 30 July 1973, NAA-NRAO, NM Operations, VLA, Design and Construction.

128. The final price of the 28 antennas was $\$ 17,755,258$. The Antenna Assembly Building cost $\$ 400,796$. NAA-NRAO, NM Operations, VLA, Design and Construction

129. The hearing was held on 30 September 1975. NAA-NRAO, NM Operations, VLA, Design and Construction.

130. House of Representatives Committee on Science and Technology Authorizing Appropriation to the National Science Foundation, HR-94-930, 18 March 1976, NAA-NRAO, NM Operations, VLA, Design and Construction.

131. A.R. Thompson to DSH, DEH, Hvatum, and Lancaster, 31 December 1975, NAA-NRAO, NM Operations, VLA, Design and Construction.

132. A.R. Thompson to DSH, DEH, H. Hvatum, and J. Lancaster, 17 February 1976, NAA-NRAO, NM Operations, VLA, Design and Construction.

133. KIK interview with WEH, 20 September 2011, NAA-KIK, Oral Interviews. https://science.nrao.edu/about/publications/open-skies\#section-7

134. Memo from Claude Kellett, NSF Program Officer for NRAO, to file, NAANRAO, NM Operations, VLA, Design and Construction.

135. NAA-NRAO, NM Operations, VLA, Design and Construction. This hearing was also attended by Robert Hughes, the NSF Assistant Director for Mathematical and Physical Sciences. Hughes later became the President of AUI, serving from 1980 to 1997.

136. Report of the Ad Hoc Advisory Panel for the Very Large Array (VLA), National Science Foundation, 31 December 1977, NAA-NRAO, NM Operations, VLA Advisory Committees, Box 2.

137. Only with the commencement of JVLA operations and the development of the VLA pipeline has it become possible to obtain a basic publishable image without a lot of labor-intensive data processing.

138. Report on the Audit Very Large Array Radio Telescope Project, NSF Office of Planning and Resources Management, Audit Office, 4 May 1977, NAANRAO, NM Operations, VLA, VLA Budget.

139. Initial members of the VLA Advisory Committee included B. Burke (MIT), J. Douglas (Univ. of Texas), F. Drake (Cornell), R. Ekers (Groningen, Netherlands), C. Heiles (Univ. Calif. Berkeley), M. Kundu (Univ. Maryland), 
A. Moffet (Caltech), G. Swenson (Univ. Illinois), A. Rogers (MIT, Haystack Observatory), R. Wilson (Bell Labs). NAA-NRAO, NM Operations, VLA Advisory Committees, Box 1.

140. Letters from MSR to VLA Advisory Committee, 18 September 1981, NAANRAO, NM Operations, VLA Advisory Committees, Box 2.

141. Members of the VLA Computer Advisory Committee were A. Brenner (Fermi National Laboratory), W. Brouw (Westerbork), P. Green (IBM), K. King (Cornell), H. S. McDonald (Bell Labs), T. Paganelli (General Electric), G.S. Patterson (Cray Laboratories), S.F. Reddaway (IBM), and H. Schorr (IBM).

142. KIK interview with CMW, 21 March 2015, op. cit.

143. Ibid.

144. See Ekers, R. 1987, The First Seven Years of VLA Operations - VLA 19801987, NAA-NRAO, NM Operations, VLA Operations. https://science.nrao. edu/about/publications/open-skies\#section-7

145. A. Moffet to DSH, 28 May 1973, NAA-NRAO, NM Operations, VLA Advisory Committees.

146. Ekers, 1987, op. cit.

147. See also AIPS Memos 61 and 87. http://library.nrao.edu/public/memos/ aips/memos/AIPSM_06l.pdf, http://library.nrao.edu/public/memos/aips/ memos/AIPSM_087.pdf

148. VLA Observational Status Reports. http://library.nrao.edu/obsstat.shtml

149. While the use of used surplus railway ties allowed NRAO to finish the VLA within the planned budget, the later operating cost of replacing the ties was greater than the initial cost saving.

150. Even earlier the WSRT provided complete hands off images for the Dutch astronomers.

151. The VLA Development Plan, Proceedings of a Science Workshop Held in Socorro, NM, 13-15 January 1995, NAA-NRAO, NM Operations, EVLA, Box 1.

152. The VLA Expansion Project, Phase I - The Ultrasensitive Array, Proposal submitted to the NSF, May 2000, NAA-NRAO, NM Operations, EVLA.

153. The Expanded VLA, Phase II, Resolving Cosmic Evolution, Completing the EVLA, 15 April 2004, NAA-NRAO, NM Operations, EVLA, Box 2.

154. J. Martuscelli to P. Vanden Bout, 4 April 2000, NAA-NRAO, NM Operations, EVLA, Box 1. CONACyT pledged to contribute 20 million Mexican Pesos (about USD I million).

155. MOU between the Hertzberg Institute of Astrophysics and the NRAO to establish a North American Program in Radio Astronomy, September 2001. NAA-NRAO, NM Operations, EVLA, Box 1. The value of the WIDAR correlator was estimated to be about USD 20 million.

156. ApJL, 20 September $2011,739$.

157. Draft of Heeschen's AAS talk is in NAA-DSH, US Radio Astronomy, History, Talks, Folder 2. 


\section{BIBLIOGRAPHY}

\section{REFERENCES}

Baars, J.W.M. et al. 1973, The Synthesis Radio Telescope at Westerbork, Proc IEEE, 61, 1258

Baars, J.W.M. and Hooghoudt, B.G. 1974, The Synthesis Radio Telescope at Westerbork. General Lay-out and Mechanical Aspects, A\& A, 31, 323

Balick, B., Bignell, C., and Hjellming, R.M. 1977, VLA Radio Maps of Four Planetary Nebulae, $B A A S, 9,601$

Basart, J.F., Miley, G.K., and Clark, B.G. 1970, Phase Measurements with an Interferometer Baseline of $11.3 \mathrm{~km}$, IEEE Trans AP, 18, 375

Becker, R.H. et al. 1995, The First Survey: Faint Images of the Radio Sky at Twenty Centimeters, ApJ, 112, 407 http://sundog.stsci.edu/top.html

Beckett, P.H. 1980, Dept. of Sociology and Anthropology Report 357 (Las Cruces: New Mexico State University)

Christiansen, W.N. and Warburton, J.A. 1955, The Distribution of Radio Brightness over the Solar Disk at a Wavelength of $21 \mathrm{~cm}$. III. The Quiet Sun. Two Dimensional Observations, Aust. J. Phys., 8, 474

Christiansen, W.N. and Högbom, J.A. 1961, The Cross Antenna of the Proposed Benelux Radio Telescope, Nature, 191, 215

Christiansen, W.N., Erickson, W.C., and Högbom, J.A. 1963a, Modification of the Design of the Proposed Benelux Radio Telescope, Nature, 197, 940

Christiansen, W.N., Erickson, W.C., and Högbom, J.A. 1963b, The Benelux Cross Antenna Project, Proc. IRE Aust., 24, 219

Clark, B.G. 1980, An Efficient Implementation of the Algorithm 'CLEAN', $A \ll A, 89,377$

Condon, J. J. et al. 1998, The NRAO VLA Sky Survey, AJ, 115, 1693

Cotton, W.D. 1979, A Method of Mapping Compact Structure in Radio Sources Using VLBI Observations, $A J, \mathbf{8 4}, 1122$

Cornwell, T.J. and Perley, R.A. eds. 1991, ASPC 19, Radio Interferometry: Theory, Techniques, and Applications, Proceedings of the 131st IAU Colloquium (San Francisco: ASP)

David, E.E., Mathews, M.V., and Noll, A.M. 2004, John R. Pierce. In Biographical Memoirs of the National Academy of Sciences, 85 (Washington: National Academy Press) http://www.nasonline.org/member-directory/deceased-members/51643.html

Dicke, R. ed. 1967, Report of the Ad Hoc Advisory Panel for Large Radio Astronomy Facilities (Washington: National Science Foundation)

Dicke, R. ed. 1969, Report of the Second Meeting of the Ad Hoc Advisory Panel for Large Radio Astronomy Facilities (Washington: National Science Foundation)

Field, G. ed. 1973a, Report of the Astrophysics and Relativity Panel. In Physics in Perspective, Vol. II, Part B (Washington: National Academy of Sciences), 771

Field, G. ed., 1973b, Report of the Astrophysics and Relativity Panel. In Astronomy and Astrophysics for the 1970s, Vol. 2 (Washington: National Academy of Sciences), 268

Findlay, J.W. and von Hoerner, S. 1972, A 65-Meter Telescope for Millimeter Wavelengths (Charlottesville: NRAO)

Florence, R. 1994, The Perfect Machine (New York: Harper Collins Publishers) 
Frater, R.H., Goss, W.M., and Wendt, H.W. 2017, Four Pillars of Radio Astronomy: Mills, Christiansen, Wild, Bracewell (Giewerbestrasse, Switzerland: Springer Nature) Greenstein, J.L. ed. 1972, Astronomy and Astrophysics for the 1970s, Vol. 1 (Washington: National Academy of Sciences), 2

Greenstein, J.L. 1984a, Optical and Radio Astronomers in the Early Years. In Serendipitous Discoveries in Radio Astronomy, ed. K.I. Kellermann and B. Sheets (Green Bank: NRAO/AUI), 79

Greenstein, J.L. 1984b, Optical and Radio Astronomers in the Early Years. In The Early Years of Radio Astronomy - Reflections Fifty Years after Jansky's Discovery, ed. W.T. Sullivan III (Cambridge: CUP), 67

Greisen, E. 1990, The Astronomical Image Processing System. In Seminar on Acquisition, Processing and Archiving of Astronomical Images, ed. G. Longo and G. Sedmak (Naples: Observatorio Astronomico di Capodimonte)

Greisen, E. 1998, Recent Developments in Experimental AIPS. In ASPC 145, Astronomical Data Analysis Software and Systems VII, ed. R. Albrecht, et al. (San Francisco: ASP), 204

Heeschen, D.S. ed. 1973, In Astronomy and Astrophysics for the 1970s, Vol. 2, ed. J. L. Greenstein (Washington: National Academy of Sciences), 2

Heeschen, D.S. 1981, The Very Large Array. In Telescopes for the 1980s, ed. G. Burbidge and A. Hewitt (Palo Alto: Annual Reviews, Inc.), 1

Heeschen, D.S. 1996, The Establishment and Early Years of NRAO, BAAS, 28, 863157

Hinder, R. and Ryle, M. 1971, Atmospheric Limitations to the Angular Resolution of Aperture Synthesis Radio Telescopes, MNRAS, 154, 229

Hjellming, R. ed. 1978, An Introduction to the Very Large Array (Socorro: NRAO)

Högbom, J.A. 1959, The Structure and Magnetic Field of the Solar Corona, PhD Dissertation, Cambridge University

Högbom, J.A. 1961, A Comparison between Two Different Designs for the Benelux Radio Telescope with Special Reference to Their Sensitivity. In Large Radio Telescopes (Paris: OECD), 131

Högbom, J.A. 1974, Aperture Synthesis with a Non-Regular Distribution of Interferometer Baselines, $A \& A S, 15,417$

Högbom, J.A. 2003, Early Work in Imaging. In ASPC 300, Radio Astronomy at the Fringe, ed. J.A. Zensus, M.H. Cohen, E. Ros (San Francisco: ASP), 17

Högbom, J.A. and Brouw, W.N. 1974, The Synthesis Radio Telescope at Westerbork. Principles of Operation, Performance and Data Reduction, $A \& A, 33,289$

Keller, G. 1961, Report of the Advisory Panel on Radio Telescopes, ApJ, 134, 927

Kraft, R.P. 2005, Jesse Greenstein, National Academy of Sciences Biographical Memoirs, 86 (Washington: National Academies Press), 1 https://www.nap.edu/read/11429/ chapter/10

Lancaster, J. 1982, Very Large Array Completion Report (Socorro: NRAO) https:// science.nrao.edu/about/publications/open-skies\#section-7

Lequeux, J. 1962, Mesures Interférométriques à Haute Résolution du Diamètre et de la Structure des Principales Radio Sources à $1420 \mathrm{MHz}$, An. Astrophys, 25, 221

Lubkin, G. B., 1975, The Decision to build the Very Large Array (Cambridge: Harvard University Press)

Maltby, P. and Moffet, A. 1962, Brightness Distribution in Discrete Radio Sources. III. The Structure of the Sources, ApJS, 7, 141

McCready, L.L., Pawsey, J.L., and Payne-Scott, R. 1947, Solar Radiation at Radio Frequencies and Its Relation to Sunspots, Proc. R. Soc. London A, 190, 357 
McMullin, J.P. et al. 2007, CASA Architecture and Applications. In ASPC 376, Astronomical Data Analysis Software and Systems XVI (San Francisco: ASP)

Napier, P.J. and Gustincic, J.J. 1977, Polarization Properties of a Cassegrain Antenna with Off-Axis Feeds and On-Axis Beam, IEEE AP-S International Symposium, 452

Noordam, J. E. and de Bruyn, A. G. 1982, High Dynamic Range Mapping of Strong Radio Sources with Application to 3C 84, Nature, 299, 597

NRAO 1965, VLA Design Report No. 1 (Green Bank: NRAO)

NRAO 1967, A Proposal for a Very Large Array Radio Telescope Vol. I and II (Green Bank: NRAO)

NRAO 1969, A Proposal for a Very Large Array Radio Telescope Vol. III (Green Bank: NRAO)

NRAO 1971, A Proposal for a Very Large Array Radio Telescope Vol. IV (Green Bank: NRAO)

NRAO 1978 Announcement, BAAS, 10 (1), 386

Oort, J.H. 1961a, Some Considerations Concerning the Study of the Universe by Means of Large Radio Telescopes. In Large Radio-Telescopes (Paris: OECD), 35

Oort, J.H. 1961b, Considerations Concerning the Minimum Resolving Power Required for $21-\mathrm{cm}$ Line Observations with a Very Large Antenna. In Large RadioTelescopes (Paris: OECD), 53

Oort, J.H. 1961c, Some Suggested Programs. In Large Radio Telescopes (Paris: OECD), 123

Perley, R. et al. 2009, The Expanded Very Large Array, Proc. IEEE, 97 (8), 1448

Perley, R. et al. 2011, The Expanded Very Large Array: A New Telescope for New Science, ApJL, 739, 1

Raimond, E. 1996, Historical Notes: Four Decades of Dutch Radio Astronomy, Twenty-Five Years Westerbork Telescope. In The Westerbork Observatory, Continuing Adventure in Radio Astronomy, ed. E. Raimond and R. Genee (Dordrecht: Kluwer), 11

Readhead, A.C.S. and Wilkinson, P.N. 1978, The Mapping of Compact Radio Sources from VLBI Data, $A p J, 223,25$

Rogstad, D.H. and Shostak, G.S. 1971, Aperture Synthesis Study of Neutral Hydrogen in the Galaxy M101: I. Observations, $A \& A, 13,99$

Ryle, M. 1962, The New Cambridge Radio Telescope, Nature, 194, 517

Ryle, M. 1972, The 5-km Radio Telescope at Cambridge, Nature, 239, 435

Ryle, M. 1975, Radio Telescopes of Large Resolving Power, Science, 188, 1071

Ryle, M. and Hewish, A. 1960, The Synthesis of Large Radio Telescopes, MNRAS, 120, 220

Ryle, M. and Neville, A.C. 1962, A Radio Survey of the North Polar Region with a 4.5 Minute of arc Pencil-Beam System, MNRAS, 125, 39

Ryle, M., Elsmore, B., and Neville, A.C. 1965, Observations of Radio Galaxies with the One-Mile Telescope at Cambridge, Nature, 194, 517

Scheuer, P.A.G. 1984, The Development of Aperture Synthesis at Cambridge. In The Early Years of Radio Astronomy, ed. W.T. Sullivan III (Cambridge: CUP), 249

Schwarz, U.J. 1978, Mathematical-Statistical Description of the Iterative Beam Removing Technique (Method CLEAN), A\&A, 65, 345

Taylor, G.B., Carilli, C.L. and Perley, R.A. eds. 1999, Synthesis Imaging in Radio Astronomy II, ASPC 180, (San Francisco: ASP)

Thompson, A.R. and D'Addario, L.A. 1982, Synthesis Mapping, Proceedings of the NRAO-VLA Workshop Held in Socorro, NM, June 21-25 (Green Bank: NRAO) 
Trimble, V. 2003, Jesse Leonard Greenstein (1909-2002), PASP, 115, 890

Trimble, V. and Zaich, P. 2006, Productivity and Impact of Radio Telescopes, PASP, 118, 933

Trimble, V. and Ceja, J.A. 2008, Productivity and Impact of Astronomical Facilities: Three Years of Publications and Citation Rates, Astron Nachr, 329, 632

Tucker, W. and Tucker, K. 1986, The Very Large Array. In The Cosmic Inquirers (Cambridge: Harvard University Press)

Whitford, A. 1964, Ground-Based Astronomy: A Ten Year Program (Washington: National Academy of Sciences)

Williams, W.F. 1965, High Efficiency Antenna Reflectors, Microwave J., 8, 79

York, D.G. et al. 2000, The Sloan Digital Sky Survey: Technical Summary, AJ, 120, 1579

\section{FURTHER READING}

Bracewell, R.N. 1958, Radio Interferometry of Discrete Sources, Proc. IRE, 46, 97

Bracewell, R.N. and Roberts, J.A. 1954, Aerial Smoothing in Radio Astronomy, Aust. J. Phys. 7, 6

Elbers, A. 2017, The Rise of Radio Astronomy in the Netherlands (Cham, Switzerland: Springer)

Finley, D.G, and Goss, W.M. eds. 2000, Radio Interferometry: The Saga and the Science: Proceedings of a Symposium Honoring Barry Clark at 60 (Washington: AUI)

Fomalont, E.B. and Wright, C.H. 1974, Interferometry and Aperture Synthesis. In Galactic and Extragalactic Radio Astronomy, ed. G. Verschuur and K.I. Kellermann (Berlin: Springer)

Heeschen, D.S. 1967, Radio Astronomy: A Large Antenna Array, Science, 158, 75

Heeschen, D.S. 1975, The Very Large Array, Sky and Telescope, 49 (6), 344

Heeschen, D. 1991, Reminiscences of the Early Days of the VLA. In ASPC 19, Radio Interferometry: Theory, Techniques, and Applications; Proceedings of the 131st IAU Colloquium, ed. T.J. Cornwell and R.A. Perley (San Francisco: ASP), 150

Hogg, D.E. 2000, The Green Bank Interferometer. In Radio Interferometry: The Saga and the Science: Proceedings of a Symposium Honoring Barry Clark at 60, ed. D.G. Finley and W. M. Goss (Washington: AUI), 20

Kellermann, K.I. 2014, David S. Heeschen. In Biographical Memoirs of the National Academy of Sciences (Washington: National Academy of Sciences) https://aas.org/ obituaries/david-s-heeschen-1926-2012

Kellermann, K.I. and Moran, J.M. 2001, The Development of High-Resolution Imaging in Radio Astronomy, $A R A \& A, 29,457$

Lancaster, J. 1991, The Design and Construction of the VLA: The Project Managers View. In ASPC 19, Radio Interferometry: Theory, Techniques, and Applications; Proceedings of the 131st IAU Colloquium, ed. T.J. Cornwell and R.A. Perley (San Francisco: ASP), 165

Leverington, D. 2017, Observatories and Telescopes of Modern Times (Cambridge: CUP)

Napier, P.J. et al. 1983, The Very Large Array: Design and Performance of a Modern Synthesis Radio Telescope, Proc. IEEE, 71, 1295

Sullivan, W.T. III 2009, Cosmic Noise: A History of Early Radio Astronomy (Cambridge: CUP)

Swenson, G. The Early Technical Development of the VLA. In ASPC 19, Radio Interferometry: Theory, Techniques, and Applications; Proceedings of the 131st IAU Colloquium, ed. T.J. Cornwell and R.A. Perley (San Francisco: ASP), 160 
Thompson, A.R. et al. 1980, The Very Large Array, ApJS, 44, 151

Thompson, A.R., Moran, J.M. and Swenson, G.W. 2017, Interferometry and Synthesis in Radio Astronomy (Cham, Switzerland: Springer) https://www.springer.com/us/ book/9783319444291

The VLA Takes Shape, SơT, 52 (5), 320

Weinreb, S. et al. 1977, Waveguide Systems for a Very Large Antenna Array, Microwave J., 20 (3), 49

Open Access This chapter is licensed under the terms of the Creative Commons Attribution 4.0 International License (http://creativecommons.org/licenses/ by/4.0/), which permits use, sharing, adaptation, distribution and reproduction in any medium or format, as long as you give appropriate credit to the original author(s) and the source, provide a link to the Creative Commons licence and indicate if changes were made.

The images or other third party material in this chapter are included in the chapter's Creative Commons licence, unless indicated otherwise in a credit line to the material. If material is not included in the chapter's Creative Commons licence and your intended use is not permitted by statutory regulation or exceeds the permitted use, you will need to obtain permission directly from the copyright holder. 